\title{
5 Computerspielabhängigkeit
}

In den vorangegangenen Kapiteln 3 (Mediennutzung und Schulleistung) sowie 4 (Gewaltmediennutzung und Gewaltdelinquenz) wurde eine problematische Mediennutzung im Sinne einer zeitlich exzessiven Mediennutzung oder einer inhaltlich problematischen Mediennutzung (etwa bei der Nutzung von Medien mit entwicklungsbeeinträchtigendem Potenzial für Minderjährige) jeweils als Erklärungsfaktor für schlechtere Schulleistungen bzw. Gewaltverhalten betrachtet. In diesem Kapitel soll die problematische Mediennutzung in Form eines suchtartigen Computerspielverhaltens, selbst zum Thema gemacht werden. So sind im Rahmen suchtartigen Mediengebrauchs zeitlich exzessive Nutzungsmuster auch mit einer individuell dysfunktionalen Mediennutzung (etwa im Sinne dysfunktionaler Stressregulation, auffälliger Substitution sozialer Interaktionen und Beziehungen durch parasoziale Interaktionen und Beziehungen zu Medienfiguren oder vehement eskapistischer Mediennutzung im Sinne einer Realitätsflucht) kombiniert (Rehbein et al., 2009b, 2010). Darüber hinaus ist aber auch zu beobachten, dass suchtartige Mediennutzung bei Kindern und Jugendlichen signifikant mit der Nutzung potenziell entwicklungsbeeinträchtigender Medieninhalte korreliert ist und zu Verzögerungen in der Entwicklung führen kann (Mößle et al., 2007; Mößle \& Kleimann, 2010).

\subsection{Theoretische Grundlagen und Literaturüberblick - Computerspielabhängigkeit}

In der Suchtforschung ist schon seit Längerem bekannt, dass eine Abhängigkeit nicht allein durch die wiederholte Einnahme einer Substanz entstehen kann (stoffgebundene Abhängigkeit). Auch wiederkehrende Verhaltensweisen können in Verbindung mit Erfolgserlebnissen und Glückserfahrungen unter bestimmten Voraussetzungen in eine stoffungebundene Abhängigkeit führen. So behandelt das menschliche Gehirn verschiedene Formen von Belohnungen ähnlich, unabhängig davon, ob diese durch einen bestimmten Stoff (z. B. Alkohol) oder eine bestimmte Verhaltensweise (z. B. Glücksspiel) ausgelöst werden (Holden, 2001). Für die Nutzung von Computerspielen konnten zum Beispiel einige physiologische Parallelen zu stoffgebundenen Abhängigkeiten aufgezeigt werden: Beide gehen mit einer erhöhten Dopaminausschüttung einher und stimulieren unmittelbar belohnungsrelevante Hirnareale (Koepp et al., 1998; vgl. für Glücksspiele, 
Meyer \& Bachmann, 2005). ${ }^{158}$ Aktuell werden in deutschsprachigen Standardwerken die Glücksspielsucht, die Kaufsucht, die Sportsucht, die Arbeitssucht, die Sexsucht sowie die Mediensucht als Formen der Verhaltenssucht diskutiert (Poppelreuter \& Groß, 2000; Grüsser, S. M. \& Thalemann, C. N., 2006; Batthyány \& Pritz, 2009), wobei bis dato lediglich das pathologische Glücksspiel Eingang in die Klassifikationen psychischer Störungen gefunden hat. Moderne digitale Bildschirmmedien, in denen multimediale, massenkommunikative Inhalte mit interaktiven Eingriffsmöglichkeiten und interpersonaler Kommunikation kombiniert werden (ein gutes Beispiel hierfür sind die sog. Massively Multiplayer Online Roleplaying Games - oder Onlinerollenspiele), können bei bestimmten Personen eine Abhängigkeitsentwicklung begünstigen und diese letztendlich, wie bei anderen Abhängigkeitserkrankungen, im engeren Sinne psychisch krank machen (vgl. te Wildt, 2009).

Die Existenz des Phänomens der pathologischen Mediennutzung konnte für das Fernsehen lediglich in Ansätzen bewiesen werden (Kubey \& Csikszentmihalyi, 2002; McIlwraith, 1998). Die Existenz und die klinische Relevanz lässt sich aber für Computerspiele und das Internet inzwischen belegen und nicht mehr anzweifeln (vgl. te Wildt, 2004; Spitzer, 2005; Bergmann \& Hüther, 2006; Grüsser, S. M. \& Thalemann, R., 2006; Griffiths, 2007; Wölfling, Thalemann \& Grüsser, 2008; Wölfling \& Müller, 2008; Batthyány \& Pritz, 2009; Wessel, Müller \& Wölfling, 2009; Müller, K. W., Glaesmer, Brähler, Wölfling \& Beutel, 2011).

Prinzipiell kann der Computer und die Kommunikationsplattform Internet eine Vielzahl von Handlungen ermöglichen, die auf den Nutzer belohnend wirken, eine exzessive Nutzung anregen und auch in einer pathologischen Nutzung münden können (vgl. te Wildt \& Rehbein, 2010). So werden hinsichtlich der Nutzung moderner interaktiver Unterhaltungsmedien auch unterschiedliche pathologische Nutzungsformen unterschieden. Young und Kollegen (Young, Pistner, O\&apos;Mara \& Buchanan, 1999) leiteten aus einer onlinegestützten Befragung von Psychotherapeuten, welche verschiedene Aspekte problematischer Mediennutzung im Rahmen ihrer Patientenkontakte beschrieben haben, die sogenannten Cyber-Disorders ab und subsumieren hierunter:

- Cybersexual Addiction (Abhängigkeit von pornografischen Angeboten im Internet)

- Cyber-relational Addiction (übermäßige Pflege von Onlinebeziehungen)

158 Alkoholiker reagieren hirnphysiologisch in ähnlicher Weise auf Bilder von Alkohol wie exzessive Computerspieler auf Bilder von Computerspielen. Eine erhöhte Aufmerksamkeit für die jeweils suchtbezogenen Reize zeigte sich für stoffgebundene Süchte und für Verhaltenssüchte in nahezu identischer Weise (Thalemann, Wölfling \& Grüsser, 2007; Thalemann \& Grüsser, 2005). 
- Net Compulsions (abhängiges Glückspiel, Kaufen und Handeln im Internet)

- Information Overload (abhängiges Surfen oder Absuchen von Datenbanken)

- Computer Addiction (inkl. abhängiges Computerspielen)

Abgesehen von der Kategorie der Net Compulsions, die inhaltlich das pathologische Glücksspiel sowie die Kaufsucht aufgreift, existieren zu den drei von Young und Kollegen (Young et al., 1999) postulierten Subtypen Cybersexual Addiction, Cyber-relational Addiction und Information Overload bislang kaum empirische Daten, die eine Einschätzung darüber erlauben würden, inwieweit aus diesen Handlungen tatsächlich eine klinisch relevante Abhängigkeit erwachsen kann.

In Anknüpfung an diese Ausgangslage sind in den letzten Jahren zunehmende und vielfältige Forschungsbemühungen zu verzeichnen. Ein erster maßgeblicher Forschungszweig widmet sich dem allgemeineren Phänomen der Internetabhängigkeit, wobei der Untersuchungsgegenstand als eine Abhängigkeit von nicht näher definierten Internetaktivitäten operationalisiert wird. Hierdurch entsteht allerdings zwangsläufig eine gewisse Unschärfe des Untersuchungsgegenstands, da nicht ersichtlich wird, welche Aktivitäten zum abhängigen Verhalten in besonderer Weise beitragen (vgl. Wölfling et al., 2008; te Wildt \& Rehbein, 2010). Zudem ist bislang nicht klar, inwieweit unterschiedliche Formen pathologischer Internetnutzung wie die Nutzung von Onlinespielen oder von Internetpornografie auch diagnostisch unterschiedlich zu bewerten sind (vgl. te Wildt \& Rehbein, 2010).

Neben dem Forschungszweig zur Internetabhängigkeit hat sich zeitlich etwas später ein inhaltlich fokussierter Forschungszweig zur Computerspielabhängigkeit entwickelt (vgl. te Wildt \& Rehbein, 2010), die aktuell als prominenteste Form der Abhängigkeit im Bereich digitaler Medien gilt und insbesondere unter männlichen Jugendlichen stark verbreitet ist. In diesem Forschungszweig ist der Untersuchungsgegenstand durch die inhaltliche Begrenzung auf Computerspiele (meist allerdings nur Online gespielte Computerspiele, wodurch die Computerspielabhängigkeit auch als Unterform der Internetabhängigkeit subsummiert werden könnte) gegenüber allgemeinen Studien zur Internetabhängigkeit, die wie aufgezeigt eine Vielzahl von Aktivitäten umfassen können, klarer definiert.

Im Folgenden soll der aktuelle Forschungsstand in den Bereichen Diagnostik (Kapitel 5.1.1), Komorbidität und Risikokorrelate (Kapitel 5.1.2), Epidemiologie (Kapitel 5.1.3) sowie unterschiedliche Erklärungsmodelle (Kapitel 5.1.4) näher dargestellt werden. 


\subsubsection{Diagnostik von Internet- und Computerspielabhängigkeit}

Die Diagnosestellung einer Internet- oder Computerspielabhängigkeit ist bis dato in den klinischen Diagnosemanualen nicht vorgesehen. Bislang fand unter den Verhaltensabhängigkeiten nur das pathologische Glücksspiel Eingang in die Nosologie, wobei es im Diagnostic and Statistical Manual of Mental Disorders (DSM-IV-TR , Saß, Wittchen, Zaudig \& Houben, 2003) unter der Rubrik ,Störungen der Impulskontrolle, nicht andernorts klassifiziert" (312; 312.31 Pathologisches Spielen) und in der Internationalen Klassifikation psychischer Störungen (ICD-10, Dilling, Mombour, Schmidt \& Schulte-Markwort, 2006) unter der Rubrik ,Abnorme Gewohnheiten und Störungen der Impulskontrolle“ (F63; F63.0 pathologisches Spielen) subsummiert wird, jeweils einer sehr heterogenen Restkategorie von Krankheitsbildern, in der u. a. auch Pyromanie und Kleptomanie zusammengefasst werden (vgl. Saß et al., 2003; Dilling et al., 2006). Medienbezogene Abhängigkeiten sind hingegen noch nicht klinisch anerkannt und werden derzeit als eine mögliche Sonderform substanzungebundener Suchterkrankungen bzw. als Verhaltenssucht (vgl. etwa Thalemann \& Grüsser, 2005) oder auch als Störung der Impulskontrolle (te Wildt, 2004), die Ausdruck anderer Erkrankungen sein könnte (Shaffer, Hall \& Vander-Bilt, 2000), diskutiert.

Zur Beurteilung der klinischen Bedeutsamkeit und der Frage, ob einem spezifischen Mediennutzungsverhalten eine psychische Abhängigkeit zugrunde liegt, wird im Rahmen aller relevanten nationalen und internationalen Forschungsbemühungen für die Diagnosestellung auf die Kriterien stoffgebundener Abhängigkeiten (vgl. Tabelle 59) oder des pathologischen Glücksspiels (vgl. Tabelle 60) zurückgegriffen. Das pathologische Glücksspiel wird in den aktuellen Klassifikationssystemen zwar als Impulskontrollstörung kategorisiert; die Definition dieses Störungsbildes fußt allerdings maßgeblich auf ein Suchtmodell, wie es auch für stoffgebundene Abhängigkeiten zugrunde gelegt wird (Meyer \& Bachmann, 2005). ${ }^{159}$

159 In der aktuellen Überarbeitung des DSM wird so auch eine Umgruppierung des pathologischen Glücksspiels aus der Kategorie „Störung der Impulskontrolle, nicht andernorts klassifiziert" unter die Suchterkrankungen diskutiert, wobei diese um die Verhaltenssüchte erweitert würden (Addiction and Related Disorders, vgl. Miller \& Holden, 2010). 

bzw. des Abhängigkeitssyndroms (ICD-10).

Substanzabhängigkeit nach DSM-IV-TR (Saß et al., 2003)

Ein unangepasstes Muster von Substanzgebrauch führt in klinisch bedeutsamer Weise zu Beeinträchtigungen oder Leiden, wobei sich mindestens drei der folgenden Kriterien manifestieren, die zu irgendeiner Zeit in demselben 12-MonatsZeitraum auftreten:

Abhängigkeitssyndrom (F1x.2) nach

$$
\text { ICD-10 }
$$

(Dilling et al., 2006)

A. Drei oder mehr der folgenden Kriterien sollen zusammen mindestens einen Monat lang bestanden haben. Falls sie nur eine kürzere Zeit gemeinsam aufgetreten sind, sollten sie innerhalb von zwölf Monaten wiederholt bestanden haben.

1. Ein starkes Verlangen oder eine Art Zwang, die Substanz zu konsumieren.

1. Toleranzentwicklung, definiert durch eines der folgenden Kriterien: (a) Verlangen nach ausgeprägter Dosissteigerung, um einen Intoxikationszustand oder gewünschten Effekt herbeizuführen, (b) deutlich verminderte Wirkung bei fortgesetzter Einnahme derselben Dosis.

2. Entzugssymptome, die sich durch eines der folgenden Kriterien äußern: (a) charakteristisches Entzugssyndrom der jeweiligen Substanz (siehe Kriterien A und B der Kriterien für Entzug von den spezifischen Substanzen, (b) dieselbe (oder eine sehr ähnliche) Substanz wird eingenommen, um Entzugssymptome $\mathrm{zu}$ lindern oder zu vermeiden.

3. Die Substanz wird häufig in größeren Mengen oder länger als beabsichtigt eingenommen.

4. Anhaltender Wunsch oder erfolglose Versuche, den Substanzgebrauch zu verringern oder zu kontrollieren.
4. Toleranzentwicklung gegenüber den Wirkungen der Substanz. Für eine Intoxikation oder um den gewünschten Effekt zu erreichen, müssen größere Mengen der Substanz konsumiert werden, oder es treten bei fortgesetztem Konsum derselben Menge deutlich geringere Effekte auf.

\section{Ein körperliches Entzugssyndrom} (siehe F1x.3 und F1x.4), wenn die Substanz reduziert oder abgesetzt wird, mit den für die Substanz typischen Entzugssymptomen oder auch nachweisbar durch den Gebrauch derselben oder einer sehr ähnlichen Substanz, um Entzugssymptome zu mildern oder zu vermeiden.

2. Verminderte Kontrolle über den Substanzgebrauch, d.h. über Beginn, Beendigung oder die Menge des Konsums, deutlich daran, dass oft mehr von der Substanz konsumiert wird oder über einen längeren Zeitraum als geplant oder an dem anhaltenden Wunsch oder an erfolgslosen Versuchen, den Substanzkonsum zu verringern oder zu kontrollieren. 
Substanzabhängigkeit nach DSM-IV-TR

(Saß et al., 2003)
Abhängigkeitssyndrom (F1x.2) nach

ICD-10

(Dilling et al., 2006)

5. Viel Zeit für Aktivitäten, um die Substanz zu beschaffen (z. B. Besuch verschiedener Ärzte oder Fahrt langer Strecken), sie zu nehmen (z. B. Kettenrauchen) oder sich von ihren Wirkungen zu erholen.

6. Wichtige soziale, berufliche oder Freizeitaktivitäten werden aufgrund des Substanzgebrauchs aufgegeben oder eingeschränkt.

5. Einengung auf den Substanzgebrauch, deutlich an der Aufgabe oder der Vernachlässigung anderer wichtiger Vergnügen oder Interessensbereiche wegen des Substanzgebrauchs; oder es wird Zeit darauf verwandt die Substanz zu beschaffen, zu konsumieren oder sich davon zu erholen.

7. Fortgesetzter Substanzgebrauch trotz Kenntnis eines anhaltenden oder wiederkehrenden körperlichen oder psychischen Problems, das wahrscheinlich durch die Substanz verursacht oder verstärkt wurde (z. B. fortgesetzter Kokaingebrauch trotz des Erkennens kokaininduzierter Depressionen oder trotz des Erkennens, dass sich ein Ulcus durch Alkoholkonsum verschlechtert).

6. Anhaltender Substanzgebrauch trotz eindeutig schädlicher Folgen (siehe F1x.1), deutlich an dem fortgesetzten Gebrauch, obwohl der Betreffende sich über die Art und das Ausmaß des Schadens bewusst ist oder bewusst sein könnte.

Anmerkung. Die Kriterien des ICD-10 wurden gemäß den Kriterien des DSM-IV-TR umstrukturiert. Die Nummerierung entspricht der Originalnummerierung. Markierungen durch den Autor.

Tabelle 60. Diagnostische Kriterien des pathologischen Spielens (DSM-IV-TR, ICD-10).

\section{Pathologisches Spielen ach DSM-IV-TR}

(Saß et al., 2003)

Pathologisches Spielen (F63.0) nach ICD-10

(Dilling et al., 2006)

A. Andauerndes und wiederkehrendes, fehlangepasstes Spielverhalten, was sich in mindestens fünf der folgenden Merkmale ausdrückt:
A. Wiederholte (zwei oder mehr) Episoden von Glücksspiel über einen Zeitraum von mindestens einem Jahr.

4. ist unruhig und gereizt beim Versuch, das Spielen einzuschränken oder aufzugeben,

6. kehrt, nachdem er/sie beim Glücksspiel Geld verloren hat, oft am nächsten Tag zurück, um den Verlust auszugleichen (dem Verlust „,hinterherjagen“)

9. hat eine wichtige Beziehung, seinen Arbeitsplatz, Ausbildungs- oder Aufstiegschancen wegen des Spielens gefährdet oder verloren,
B. Diese Episoden bringen den Betroffenen keinen Gewinn, sondern werden trotz subjektivem Leidensdruck und Störungen der Funktionsfähigkeit im täglichen Leben fortgesetzt. 
C. Die Betroffenen betreiben einen intensiven Drang, zu spielen,

3. hat wiederholt erfolglose Versuche unternommen, das Spielen zu kontrollieren, einzuschränken oder aufzugeben, der nur schwer kontrolliert werden kann. Sie schildern, dass sie nicht in der Lage sind, das Glücksspiel durch Willensanstrengung zu unterbrechen.

1. ist stark eingenommen vom Glücksspiel (z. B. starkes Beschäftigtsein mit gedanklichen Nacherleben vergangener Spielerfahrungen, mit Verhindern oder Planen der nächsten Spielunternehmungen, Nachdenken über Wege, Geld zum Spielen zu beschaffen),

D. Die Betroffenen sind ständig mit Gedanken oder Vorstellungen vom Glücksspiel oder mit dem Umfeld des Spiels beschäftigt.

2. muss mit immer höheren Einsätzen spielen, um die gewünschte Erregung zu erreichen,

5. spielt, um Problemen zu entkommen oder um eine dysphorische Stimmung (z. B. Gefühle von Hilflosigkeit, Schuld, Angst, Depression) zu erleichtern,

7. belügt Familienmitglieder, den Therapeuten oder andere, um das Ausmaß der Verstrickung in das Spielen zu vertuschen,

8. hat illegale Handlungen wie Fälschung, Betrug, Diebstahl oder Unterschlagung begangen, um das Spielen zu finanzieren,

10. verlässt sich darauf, dass andere Geld bereitstellen, um die durch das Spielen verursachte hoffnungslose finanzielle Situation zu überwinden.

B. Das Spielverhalten kann nicht besser durch eine Manische Episode erklärt werden.

Anmerkung. Die Kriterien des DSM-IV-TR wurden gemäß den Kriterien des ICD-10 umstrukturiert. Die Nummerierung entspricht der Originalnummerierung. Markierungen durch den Autor.

Diagnostische Kriterien. Bereits Mitte der 1990er wurden die diagnostischen Kriterien des pathologischen Spielens (DSM-IV) erstmalig von Young auf den Bereich der Internetabhängigkeit (Internet Addiction) übertragen und angewandt, wobei nur acht der zehn Diagnosekriterien des pathologischen Spielens Verwendung fanden (Young, 1998). „Illegale Handlungen zur Finanzierung des Spie- 
lens“ sowie „Hoffnung auf Bereitstellung von Geld durch Dritte“ wurden aufgrund einer fehlenden Passung zum Gegenstandsbereich nicht übernommen. Operationalisiert wurde Internet Addiction dabei von Young (1998) über ein kurzes Screeninginstrument (Diagnostic Questionnaire - DQ Internet Addiction), welches die folgenden acht dichotomen (Ja/Nein) Fragen beinhaltete:

1. Do you feel preoccupied with the Internet (think about previous on-line activity or anticipate next on-line session)? (starke Eingenommenheit)

2. Do you feel the need to use the Internet with increasing amounts of time in order to achieve satisfaction? (Steigerung der Einsätze)

3. Have you repeatedly made unsuccessful efforts to control, cut back, or stop Internet use? (Kontrollverlust)

4. Do you feel restless, moody, depressed, or irritable when attempting to cut down or stop Internet use? (Unruhe und Gereiztheit)

5. Do you stay on-line longer than originally intended? (Wiederaufnahme)

6. Have you jeopardized or risked the loss of significant relationship, job, educational or career opportunity because of the Internet? (Gefährdung oder Verlust)

7. Have you lied to family members, therapist, or others to conceal the extent of involvement with the Internet? (Lügen)

8. Do you use the Internet as a way of escaping from problems or of relieving a dysphoric mood (e.g., feelings of helplessness, guilt, anxiety, depression)? (Stimmungsregulation)

Trotz einer Verkürzung um zwei Kriterien wurde analog zum pathologischen Spielen der kritische Wert von fünf Ja-Antworten zur Bestimmung einer Internetabhängigkeit beibehalten.

$\mathrm{Zu}$ Beginn des neuen Jahrtausends wurden diese diagnostischen Kriterien von Beard und Wolf (2001) dahingehend verschärft, dass zunächst diejenigen Kriterien, welche sich ausschließlich auf eine pathologische Internetnutzung beziehen (1 - 5), allesamt erfüllt sein müssen, um die Diagnose Internetabhängigkeit zu stellen. Zusätzlich muss aber auch mindestens eines der Kriterien, welche Folgeschäden der pathologischen Internetnutzung beschreiben $(6-8)$, mit Ja beantwortet werden. Beard und Wolf (2001) begründeten diese Verschärfung damit, dass die ersten fünf Kriterien auch bei zahlreichen anderen Verhaltensweisen, die nicht notwendigerweise als Abhängigkeit klassifiziert werden müssten, in dieser 
Form beobachtet werden könnten ${ }^{160}$ und durchaus noch mit einem geregelten Alltagsleben vereinbar seien. Erst die letzten drei Kriterien würden auf eine wirklich pathologische Nutzung hindeuten, da der pathologische Internetnutzer in seiner Funktionsfähigkeit sowie im Zusammenleben eingeschränkt sei.

Diagnostische Kriterien zur Bestimmung einer Internetabhängigkeit speziell im Jugendalter wurden 2005 von einer Südkoreanischen ${ }^{161}$ Forschergruppe vorgeschlagen (Ko, C. H., Yen, Chen, Chen \& Yen, 2005). Internetabhängigkeit im Jugendalter wurde dabei über folgenden Kriterienkatalog (DC-IA-A; Ko, C. H. et al., 2005) operationalisiert, der Diagnosekriterien des Pathologischen Spielens sowie der Substanzabhängigkeit nach DSM-IV-TR (Saß et al., 2003) vereint: ${ }^{162}$

A maladaptive pattern of Internet use, leading to clinically significant impairment or distress, occurring at any time within the same 3-month period.
A. Six (or more) of the following symptoms have been present
1. Preoccupation with Internet activities
2. Recurrent failure to resist the impulse to use the Internet
3. Tolerance: a marked increase in the duration of Internet use needed to achieve satisfaction

160 "For example, a new mother may be preoccupied with thoughts of her new baby (criteria 1). She may desire increased amount of time with her child (criteria 2). She has unsuccessfully been able to control, cut back, or stop her interactions with her child (criteria 3). She may feel restless, moody, depressed, or irritable when she leaves the child with a family member or at a day-care center (criteria 4). Finally, she may interact with the child for longer periods of time than originally intended, such as planning on rocking the child until the child sleeps, but the mother continues to rock (criteria 5). Would we say that this new mother is addicted to her newborn child?" (Beard \& Wolf, 2001, S. 380) Beard und Wolf erbringen somit den berechtigten Einwand einer überproportionalen Pathologisierung menschlichen Verhaltens.

161 Südkorea, ein Land mit insgesamt sehr hohen Mediennutzungszeiten, insbesondere im Bereich der Computerspiele und vor allem der Onlinespiele, kann als einer der Vorreiter der Erforschung und Therapie der Internet- oder Computerspielabhängigkeit betrachtet werden. So gib es dort bereits eine breite Versorgungslandschaft mit differenzierten Behandlungsangeboten zur Therapie von computerspiel- oder internetabhängigen Jugendlichen und jungen Erwachsenen (vgl. Ahn, 2007). Erste Präventions- und Interventionsprogramme gegen Computer- und Internetabhängigkeit wurden im schulischen Kontext initiiert (Ju, 2007). Nach Angaben der Korean Academy of Child and Adolescence Psychiatry (KADO) weisen etwa drei Prozent der zehn Millionen Kinder und Jugendlichen in Korea (also ca. 300.000) eine Internet- oder Computerspielabhängigkeit mit klinischer Behandlungsbedürftigkeit auf. Weitere 15 Prozent (1.5 Millionen), die als gefährdet klassifiziert werden, benötigen der Studie zufolge professionelle Beratungsangebote (vgl. Ahn, 2007). Als Folgen werden ernsthafte Schwierigkeiten im familiären Umfeld und in der Schule, Schlafmangel, Persönlichkeits- sowie dissoziative Veränderungen berichtet, die allesamt die soziale Entwicklung und das soziale Miteinander beeinträchtigen (vgl. Ahn, 2007).

162 Dies ist vor allem der Ermangelung einer eigenen Kategorie „Verhaltenssucht“ und der Unterordnung des pathologischen Glücksspiels unter „Störungen der Impulskontrolle, nicht andernorts klassifiziert" im DSM-IV-TR trotz großer Überschneidungen mit der Substanzabhängigkeit geschuldet. 
4. Withdrawal, as manifested by either of the following:

i. Symptoms of dysphoric mood, anxiety, irritability, and boredom after several days without Internet activity

ii. Use of Internet to relieve or avoid withdrawal symptoms

5. Use of Internet for a period of time longer than intended

6. Persistent desire and/or unsuccessful attempts to cut down or reduce Internet use

7. Excessive time spent on Internet activities and leaving the Internet

8. Excessive effort spent on activities necessary to obtain access to the Internet

9. Continued heavy Internet use despite knowledge of having a persistent or recurrent physical or psychological problem likely to have been caused or exacerbated by Internet use.

B. Functional impairment: one (or more) of the following symptoms have been present

1. Recurrent Internet use resulting in a failure to fulfill major role obligations at school and home

2. Impairment of social relationships

3. Behavior violating school rules or laws due to Internet use

C. The Internet addictive behavior is not better accounted for by psychotic disorder or bipolar I disorder, or other disorder, which is classified in impulse control disorder and paraphilia in DSM-IV-TR.

Die Diagnosestellung verläuft demgemäß in drei Schritten: Innerhalb der letzten 3 Monate müssen zunächst mindestens sechs von neun ausformulierten Diagnosekriterien (Starke Eingenommenheit $(1,6)$, Kontrollverlust $(2,5,6)$, Toleranzentwicklung (3), Entzugssymptome (4), Einengung auf den Subtanzgebrauch (7, 8) negative Folgen (9)) vorgelegen haben. Zudem muss im selben Zeitraum mindestens eine von drei ausformulierten funktionellen Beeinträchtigungen vorgelegen haben und das abhängige Internetverhalten darf für diesen Zeitraum differenzialdiagnostisch auch nicht durch andere psychische Erkrankungen besser erklärt werden können.

Wie beim pathologischen Glücksspiel wird von Ko und Kollegen (2005) somit dem Kontrollverlust, der hier mit zwei bzw. drei Diagnosekriterien erhoben wird, auch für die Diagnose von Internetabhängigkeit eine zentrale Rolle zugesprochen. Zudem werden analog zur Substanzabhängigkeit nach DSM-IV-TR (Saß et al., 2003) die Entzugssymptome aufgespalten nach einem Erleben derselben während einer internetlosen Zeit und nach einer Nutzung des Internets um diese zu unterdrücken. Im Gegensatz zu den Diagnosekriterien nach Young 
(1998) und deren Weiterentwicklung nach Beard und Wolf (2001) werden die beiden Diagnosekriterien ,Spielen, um Problemen oder negativen Stimmungen zu entkommen“ und „Lügen gegenüber Dritten, um das Ausmaß der Spielproblematik zu vertuschen" des pathologischen Spielens nach DSM-IV-TR ( $\mathrm{Sa}$ et al., 2003) nicht berücksichtigt. Dagegen wird den funktionellen Beeinträchtigungen durch ihre getrennte Auflistung ähnlich wie bei Beard und Wolf (2001) auch eine notwendige Rolle zur Diagnose von Internetabhängigkeit zugewiesen. Sie sind aber im Vergleich deutlich stärker ausdifferenziert und insbesondere auf den (schulischen) Kontext der Jugendlichen übertragen. Durch das ebenfalls notwendigerweise zu erfüllende Zeitkriterium werden kurzzeitige Episoden exzessiven Spielens, die durchaus auch kurzfristige Beeinträchtigungen mit sich bringen können, nicht fälschlicherweise als eine Internetabhängigkeit klassifiziert. Zudem wird mit dem letzten Punkt eine Berücksichtigung möglicher Differenzialdiagnosen auch bei der Diagnose Internetabhängigkeit sichergestellt (vgl. Punkt B. der Kriterien pathologischen Spielens nach DSM-IV-TR (Saß et al., 2003)).

Im Abgleich mit dem klinischen Eindruck weist das Verfahren insgesamt gute klinische Validität auf (Ko, C. H. et al., 2005). Die Anwendbarkeit des Kriterienkataloges konnte zudem auch für eine ältere Stichprobe von Collegestudenten nachgewiesen werden (Ko, C. H. et al., 2009).

Diagnoseinstrumente zur Internetabhängigkeit. Auf Grundlage der Vorüberlegungen von Young (1998) wurden parallel zur Weiterentwicklung der diagnostischen Kriterien international psychometrische Testinstrumente zur Diagnose von Internetabhängigkeit und Computerspielabhängigkeit (siehe unten), in denen sich zum Teil die Überlegungen von Ko und Kollegen (Ko, C. H. et al., 2009) ebenfalls widerfinden, entwickelt. Der am besten evaluierte und am häufigsten verwendete deutschsprachige Fragebogen ist die Internetsuchtskala (ISS) von Hahn und Jerusalem (2001b, 2001c, 2001a, 2010). Die ISS orientiert sich dabei an den Kriterien der Substanzabhängigkeit nach DSM-IV-TR ( $\mathrm{Sa} ß$ et al., 2003) und beinhaltet die Skalen Kontrollverlust, Entzugserscheinungen, Toleranzentwicklung, negative Konsequenzen Arbeit und Leistung sowie negative Konsequenzen soziale Beziehungen (Tabelle 61). Das Instrument besteht aus 20 Items und weist mit einem Cronbachs Alpha von.93 eine hohe Zuverlässigkeit und unter Einschränkungen (Subskala Toleranzentwicklung) eine gute Konstruktvalidität auf (Hahn \& Jerusalem, 2010). Zur Diagnosestellung werden die einzelnen Items aufsummiert: Personen mit Summenwerten größer als 59 werden als internetsüchtig, Personen mit Summenwerten zwischen 50 und 59 als internetsuchtgefährdet eingestuft. 
Tabelle 61. Internetsuchtskala (ISS) von Hahn und Jerusalem (2001b, 2001c, 2001a, 2010).

trifft nicht zu (1), trifft kaum zu (2), trifft eher zu (3), trifft genau zu (4)

\section{Kontrollverlust}

1. Beim Internet-Surfen ertappe ich mich häufig dabei, dass ich sage: Nur noch ein paar Minuten, und dann kann ich doch nicht aufhören.

2. Ich verbringe oft mehr Zeit im Internet, als ich mir vorgenommen habe.

3. Ich habe schon häufiger vergeblich versucht, meine Zeit im Internet zu reduzieren.

4. Ich gebe mehr Geld für das Internet aus, als ich mir eigentlich leisten kann.

\section{Entzugserscheinungen}

5. Ich beschäftige mich auch während der Zeit, in der ich nicht das Internet nutze, gedanklich sehr viel mit dem Internet.

6. Meine Gedanken kreisen ständig um das Internet, auch wenn ich gar nicht im Netz bin.

7. Wenn ich längere Zeit nicht im Internet bin, werde ich unruhig und nervös.

8. Wenn ich nicht im Internet sein kann, bin ich gereizt und unzufrieden.

\section{Toleranzentwicklung}

9. Mittlerweile verbringe ich mehr Zeit im Internet als zu Beginn meiner Online-Aktivitäten.

10. Die Zeit, die ich im Internet verbringe, hat sich im Vergleich zur Anfangszeit ständig erhöht.

11. Mein Verlangen danach, mehr Zeit im Internet zu verbringen, hat sich im Vergleich $\mathrm{zu}$ früher ständig erhöht.

12. Mein Alltag wird zunehmend stärker durch Internet-Aktivitäten bestimmt.

\section{Negative Konsequenzen Arbeit und Leistung}

13. Ich bin so häufig und intensiv mit dem Internet beschäftigt, dass ich manchmal Probleme mit meinem Arbeitgeber oder in der Schule bekomme.

14. Meine Leistungen in der Schule/im Beruf leiden unter meiner Internet-Nutzung.

15. Ich vernachlässige oft meine Pflichten, um mehr Zeit im Internet verbringen zu können.

16. Wegen des Internets verpasse ich manchmal wichtige Termine/Verabredungen.

\section{Negative Konsequenzen soziale Beziehungen}

17. Mir wichtige Menschen sagen, dass ich mich zu meinen Ungunsten verändert habe, seitdem ich das Netz nutze.

18. Seitdem ich das Internet nutze, haben sich einige Freunde von mir zurückgezogen.

19. Mir wichtige Menschen beschweren sich, dass ich zu viel Zeit im Netz verbringe.

20. Seitdem ich die Online-Welt entdeckt habe, unternehme ich weniger mit anderen.

Internetsuchtgefährdet: Summenwert 50 - 59; Internetsüchtig: Summenwert > 59

Eine aktuellere Konzeption zur Erfassung von Internetabhängigkeit stellt die Compulsive Internet Use Scale (CIUS) von Meerkerk und Kollegen (Meerkerk et al., 2009) dar, die sich in ähnlicher Weise wie frühere Instrumente (vgl. ISS; Hahn \& Jerusalem, 2001b, 2001c, 2001a) an die Kernkriterien einer Substanzab- 
hängigkeit nach DSM-IV-TR (Saß et al., 2003) anlehnt und dabei die Dimensionen Entzugserscheinungen, Kontrollverlust, Einengung des Denkens und Verhaltens, negative Konsequenzen und Stressregulation/Coping berücksichtigt (vgl. Tabelle 62). Das Instrument besteht aus 14 Items und weist mit einem über mehrere Untersuchungen aufgezeigten Cronbachs Alpha um.90 eine gute interne Konsistenz auf. Cut-Off-Werte werden leider nicht berichtet. Erste konvergente Validitätsmaße liegen im Sinne von Zusammenhangsmaßen mit anderen Instrumenten zur Internetabhängigkeit, der Internetnutzungszeit sowie eines Items der Selbsteinschätzung zur Internetabhängigkeit vor (Meerkerk et al., 2009).

Tabelle 62. Compulsive Internet Use Scale (CIUS) von Meerkerk und Kollegen (Meerkerk et al., 2009).

\section{How often (never - 0, seldom - 1, sometimes - 2, often - 3, very often - 4)...}

1. Do you find it difficult to stop using the Internet when you are online?

2. Do you continue to use the Internet despite your intention to stop?

3. Do others (e.g., partner, children, parents) say you should use the Internet less?

4. Do you prefer to use the Internet instead of spending time with others (e.g., partner, children, parents)?

5. Are you short of sleep because of the Internet?

6. Do you think about the Internet, even when not online?

7. Do you look forward to your next Internet session?

8. Do you think you should use the Internet less often?

9. Have you unsuccessfully tried to spend less time on the Internet?

10. Do you rush through your (home) work in order to go on the Internet?

11. Do you neglect your daily obligations (work, school, or family life) because you prefer to go on the Internet?

12. Do you go on the Internet when you are feeling down?

13. Do you use the Internet to escape from your sorrows or get relief from negative feelings?

14. Do you feel restless, frustrated, or irritated when you cannot use the Internet?

Eine weitere aktuelle Konzeption zur Erfassung von Internetabhängigkeit im Erwachsenenalter ist die Skala zum Onlinesuchtverhalten bei Erwachsenen (OSVeS) von Wölfling, Müller, K.W. und Beutel (2010a), die alle Kernkriterien einer Substanzabhängigkeit nach DSM-IV-TR (Saß et al., 2003) abdeckt und zusätzlich ein starkes Verlangen nach den Diagnosekriterien des Abhängigkeitssyndroms nach ICD-10 (Dilling et al., 2006) sowie Spielen, um Problemen oder negativen Stimmungen zu entkommen aus den Diagnosekriterien des pathologischen Spielens nach DSM-IV-TR (Saß et al., 2003) enthält. Da die OSVe-S allerdings eine für Erwachsene verallgemeinerte Weiterentwicklung der Skala zum Computerspielsuchtverhalten (CSV-S, vgl. Tabelle 64) darstellt, welche später ausführlich beschrieben werden soll, wird auf diese hier nicht extra eingegangen (tauscht man in der CSV-S Computerspiele durch Onlineaktivitäten oder online und ersetzt $\mathrm{Du}$ durch Sie, erhält man die OSVe-S). Die Einstufung pathologi- 
schen Nutzungsverhaltens erfolgt analog. In Ergänzung zur CSV-S werden allerdings in der Version zum Onlineverhalten zusätzlich einige demografische Variablen sowie das spezifische Onlinenutzungsverhalten (Onlinespiele, Einkaufen, Chatten, Emails schreiben, Onlinesex-Angebote, Online-Glücksspiele, OnlineCommunities, Informationsrecherche) ${ }^{163}$ erhoben. Erste Überprüfungen der Validität und Reliabilität weisen die OSVe-S als brauchbares und standardisiertes Instrument zur Klassifikation pathologischen Internetnutzungsverhaltens aus (Müller, K. W. \& Wölfling, 2010).

Als problematisch muss bei den aufgeführten Instrumenten zur Internetabhängigkeit gelten, dass sie keine zuverlässige Aussage über das zugrundeliegende Mediennutzungsverhalten ermöglichen, da jeweils unklar bleibt, welche Internetaktivitäten das abhängige Verhalten bestimmen. Die Umsetzung in der OSVe-S von Wölfling, Müller und Beutel (2010a) kann hier nur als erster Versuch der differenzierten Erfassung gewertet werden, da hier z. B. bei der Nennung mehrerer Online-Angebote auf nicht das dominierende Verhalten rückgeschlossen werden kann. Gleichzeitig können Instrumente zur Internetabhängigkeit auch nicht für sich beanspruchen, alle hinsichtlich ihres Abhängigkeitspotenzials relevanten Medienaktivitäten hinreichend abzudecken. So werden Offline-Computeraktivitäten wie die Nutzung von Computerspielen ohne Internetanbindung grundsätzlich aus der Diagnostik ausgeschlossen, obgleich diese nachweislich ebenfalls von abhängigen Spielern genutzt werden (Rehbein et al., 2009a, 2009b, 2010). Darüber hinaus könnte sich auch für Nutzer von Online-Computerspielen zeigen, dass diese ihr Spielverhalten weniger als Internetnutzung sondern vielmehr als Computerspielnutzung verstehen, wodurch weitere Bedenken bezüglich der inhaltlichen Validität der Verfahren aufgeworfen werden. Zudem lässt die aus dem Einsatz einer globalen Internetskala resultierende Unschärfe des erfassten Problemgegenstandes in der Folge befürchten, dass auch die fundierte Herleitung eines diagnostischen und für die klinische Praxis relevanten Grenzwertes (Cut-offWert), bei dem konsensual von einer pathologischen Nutzung ausgegangen wird, kaum geleistet werden kann. So ist anzunehmen, dass in der diagnostischen Urteilsfindung auffällig erhöhte Werte in Zusammenhang mit dem Chatten, der Nutzung pornografischer Inhalte oder dem Computerspielen diagnostisch jeweils anders zu bewerten und zu gewichten sind, um ein pathologisches Verhalten zu begründen, womit je nach Verhaltensweise auch eventuell unterschiedliche Cutoff-Werte zugrunde gelegt werden müssen (vgl. Rehbein, Mößle, Jukschat \& Zenses, 2011; te Wildt \& Rehbein, 2010).

Diagnoseinstrumente zur Computerspielabhängigkeit. Diagnoseinstrumente zur Computerspielabhängigkeit lehnen sich zum Teil in ähnlicher Weise

163 Somit werden die Cyber-Disorders nach Young und Kollegen (Young et al., 1999) etwas differenzierter erhoben. 
an die Diagnosekriterien einer Substanzabhängigkeit nach DSM-IV-TR ( $\mathrm{Sa}$ et al., 2003) oder das Abhängigkeitssyndrom nach ICD-10 (Dilling et al., 2006) an, verweisen im Rahmen der Befragung jedoch explizit auf das infrage stehende Spielverhalten. Auffällig ist zudem, das Skalen zur Erfassung von Computerspielabhängigekit zunächst an und für kindliche oder jugendliche Stichproben entwickelt wurden. Ein erstes Diagnoseinstrument für Computerspielabhängigkeit im Kindesalter wurde in Deutschland in der Forschergruppe um Grüsser entwickelt (Thalemann, Albrecht, Thalemann \& Grüsser, 2004; Grüsser, Thalemann, Albrecht \& Thalemann, 2005; Wölfling et al., 2008). Bei der Entwicklung des Fragebogen zum Computerspielverhalten bei Kindern (CSVK) orientierte sich diese Forschergruppe an den Kernkriterien einer Substanzabhängigkeit nach DSM-IV-TR (Saß et al., 2003), wobei wiederum ein starkes Verlangen nach den Diagnosekriterien des Abhängigkeitssyndroms nach ICD-10 (Dilling et al., 2006) mit aufgenommen wurde. Grüsser und Kollegen weisen aber berechtigterweise darauf hin, „, dass für das Kindesalter [...] Formulierungen wie „nichtstoffgebundene Abhängigkeit“" jedoch nur mit Vorsicht verwendet werden [sollten], obwohl sich hierzu teilweise Thesen in der Literatur finden lassen" (Grüsser et al., 2005, S. 193). Der CSVK (Thalemann et al., 2004; Grüsser et al., 2005) beinhaltete in seiner Ursprungsform insgesamt sieben diagnosebezogene Items eines exzessiven Spielverhaltens. Die ersten beiden Items erfassen Häufigkeit und Dauer, die weiteren fünf problematische Aspekte des Spielens. Kinder, die hinsichtlich aller sieben Merkmale überdurchschnittliche Werte aufwiesen, wurden als exzessive Spieler klassifiziert. In der weiterentwickelten Fassung des Fragebogens, der CSVK-R von Wölfling, Thalemann und Grüsser (2008), der in Items und Auswertung durchgängig vom CSVK abweicht, werden lediglich sechs diagnostische Kriterien abgefragt, von denen für eine Diagnosestellung mindestens drei erfüllt sein müssen (siehe Tabelle 63) Ein mögliches pathologisches Spielverhalten wird jedoch nur bei regelmäßiger Spielnutzung (mindestens 2-3 Mal pro Woche) angenommen. 
Tabelle 63. Fragebogen zum Computerspielverhalten von Kindern (CSVK-R, Wölfling et al., 2008).

1. Wie stark ist Dein durchschnittliches Verlangen nach Computerspielen? (1-10, gar kein Verlangen - sehr starkes Verlangen, Kriterium erfüllt wenn > 7)

2. Hast Du häufiger länger gespielt als Du beabsichtigt hast? (0-3, nie-ja immer, Kriterium erfüllt wenn meistens (2) oder häufiger)

3. Sind aufgrund Deines Computerspielverhaltens negative Folgen/Probleme in folgenden Bereichen aufgetreten? (Schulprobleme, familiäre Probleme, Geldprobleme, Vernachlässigung anderer Freizeitaktivitäten, Vernachlässigung von Freunden, Probleme mit deiner Gesundheit)

(Kriterium erfüllt wenn mindestens zwei Problembereiche bejaht wurden)

4. Hast Du Wichtiges (z. B. Hausaufgaben) vergessen, weil Du die ganze Zeit Computerspiele gespielt hast?

(0-3, nie-ja immer, Kriterium erfüllt wenn meistens (2) oder häufiger)

5. Hast Du bemerkt, dass Du immer häufiger oder länger spielen musst, um Dich wieder gut oder entspannt zu fühlen?

(0-3, nie - oft, Kriterium erfüllt wenn meistens (2) oder manchmal)

6. Fühlst Du dich schlecht, wenn Du nicht spielen kannst?

(1-10, gar kein Verlangen - sehr starkes Verlangen, Kriterium erfüllt wenn > 7)

Symptomkomplex des psychopathologischen Computerspielverhaltens erfüllt, wenn mindestens 3 von 6 Kriterien zutreffen.

In Form der Skala zum Computerspielverhalten (CSV-S) wurde der Fragebogen schließlich von Wölfling, Müller und Beutel (2010b) für eine jugendliche Stichprobe weiterentwickelt. Wiederum weicht diese in Items und Auswertung durchgängig vom CSVK-R ab. Als zusätzliches Diagnosekriterium wurde neben den oben genannten noch Spielen, um Problemen oder negativen Stimmungen zu entkommen aus den Diagnosekriterien des pathologischen Spielens nach DSM-IVTR (Saß et al., 2003) in die Skala mitaufgenommen (Tabelle 64). Das Instrument besteht aus 15 Items und weist mit einem Cronbachs Alpha von im Mittel.94 eine gute Zuverlässigkeit und eine hohe Konstruktvalidität auf (2010b). Zur Diagnosestellung werden die einzelnen Items gewichtet und aufsummiert (vgl. Tabelle 64): Personen mit Summenwerten größer als 13 werden als computerspielsüchtig, Personen mit Summenwerten zwischen 7 und 13 als Exzessivnutzer eingestuft. 
Tabelle 64. Fragebogen zum Computerspielverhalten (CSV-S, Wölfling et al., 2010b).

Im Folgenden findest du einige Fragen dazu, wie du im Allgemeinen mit Computerspielen umgehst. Bitte beantworte alle Fragen so wahrheitsgemäß und vollständig wie möglich. Denke bitte daran, dass es keine richtigen oder falschen Antworten gibt. Überlege bei der Beantwortung der einzelnen Fragen also nicht allzu lange, sondern kreuze diejenige Antwort an, welche für dich als am besten passend erscheint.

1. Wie viele Stunden spielst du durchschnittlich pro Wochentag? (offenes Antwortformat, Kriterium ab Angabe 4 Stunden, 1 Punkt)

2. Wie viele Stunden spielst du durchschnittlich pro Tag am Wochenende? (offenes Antwortformat, ohne Wertung)

3. Wie häufig spielst du Computerspiele? $(1$ = weniger als einmal pro Monat, 2 = einmal pro Monat, 3 = einmal pro Woche, 4 = 2-3 Mal pro Woche, 5 = jeden Tag; Kriterium jeden Tag, 2 Punkte)

4. Wie lange spielst du in der Regel?

( 1 = weniger als eine Stunde, $2=1-2$ Stunden, $3=2-4$ Stunden, $4=4-6$ Stunden, $5=$ mehr als 6 Stunden; Kriterium 4-6 Stunden, 1 Punkt, Kriterium mehr als 6 Stunden, 2 Punkte)

5. Wie stark bist du am Tag gedanklich mit Computerspielen beschäftigt? $(0=$ gar nicht, 1 = etwas, $2=$ merklich, $3=$ stark, $4=$ sehr stark; Kriterium stark, 1 Punkt, Kriterium sehr stark, 2 Punkte)

6. Wie häufig hast du gespielt, obwohl du dir vorgenommen hattest, nicht zu spielen? $(0=$ nie, 1 = selten, 2 = gelegentlich, 3 = oft, $4=$ sehr oft; Kriterium oft, 1 Punkt, Kriterium sehr oft, 2 Punkte)

7. Fühlst du dich schlecht, wenn du keine Computerspiele spielen kannst? $(0=$ nie, 1 = selten, 2 = gelegentlich, $3=$ oft, $4=$ sehr oft; Kriterium oft oder gelegentlich, 1 Punkt, Kriterium sehr oft, 2 Punkte)

8. Hast du bemerkt, dass du immer länger spielen musst, um dich gut zu fühlen? $(0=$ nie, 1 = selten, 2 = gelegentlich, $3=$ oft, $4=$ sehr oft; Kriterium oft, 1 Punkt, Kriterium sehr oft, 2 Punkte)

9. Wie stark ist dein durchschnittliches Verlangen nach Computerspielen? $(0=$ gar nicht, 1 = etwas, 2 = merklich, $3=$ stark, $4=$ sehr stark; Kriterium stark, 1 Punkt, Kriterium sehr stark, 2 Punkte)

10. Wie oft erscheint dir das Verlangen nach Computerspielen unkontrollierbar groß? $(0=$ nie, 1 = selten, 2 = gelegentlich, 3 = oft, $4=$ sehr oft; Kriterium sehr oft, 1 Punkt)

11. Wie oft vermeidest du negative Gefühle durch Computerspiele? $(0=$ nie, $1=$ selten, 2 = gelegentlich, $3=$ oft, $4=$ sehr oft; Kriterium oft, 1 Punkt, Kriterium sehr oft, 2 Punkte)

12. A) Wie oft hast du versucht, das Computerspielen aufzugeben / einzuschränken? B) Hast du es geschafft, das Computerspielen aufzugeben / einzuschränken? ( $\mathrm{Ja} / \mathrm{Nein})$ $(0=$ nie, $1=$ selten, 2 = gelegentlich, $3=$ oft, $4=$ sehr oft ; Kriterium oft und Nein, 2 Punkte, Kriterium sehr oft und Nein, 2 Punkte)

13. Wie oft hast du etwas Wichtiges Vergessen, weil du am Computer gespielt hast? $(0=$ nie, 1 = selten, 2 = gelegentlich, $3=$ oft, $4=$ sehr oft; Kriterium oft, 1 Punkt, Kriterium sehr oft, 2 Punkte)

14. Wie oft hattest du das Gefühl, zu lange oder zu oft gespielt zu haben? $(0=$ nie, 1 = selten, $2=$ gelegentlich, $3=$ oft, $4=$ sehr oft; Kriterium oft, 1 Punkt, Kriterium sehr oft, 2 Punkte) 
15. Sind aufgrund deines Spielverhaltens negative Konsequenzen aufgetreten?

(Insgesamt 6 Nennungen; 0 = nicht zutreffend, 1 = zutreffend; pro negativer Konsequenz 0,5$)$

Maximal erreichbare Punktzahl 27 Punkte; 7 bis 13 Punkte exzessive Nutzer, ab 14 Punkten suchtartige Nutzer.

Ein weiteres deutschsprachiges Diagnoseinstrument (vgl. Tabelle 69), ist die für die KFN Schülerbefragung 2007/2008 weiterentwickelte Computerspielabhängigkeitsskala (KFN-CSAS-II, vgl. Mößle et al., 2007). Diese basiert auf der Internetsuchtskala (ISS) von Hahn und Jerusalem (2001b, 2001c, 2001a, 2010), lehnt sich an die bestehende Abhängigkeitsklassifikation des ICD-10 (Dilling et al., 2006) an und wurde speziell für eine jugendliche Stichprobe entwickelt (zu den Hintergründen der Entwicklung vgl. auch Rehbein et al., 2009a, 2009b, 2010; Rehbein \& Borchers, 2009). Erhoben werden die Dimensionen Einengung des Denkens und Verhaltens, Negative Konsequenzen, Kontrollverlust, Entzugserscheinungen und Toleranzentwicklung. Alle Items weisen dem Anwendungszweck entsprechend eine insgesamt hohe Itemschwierigkeit und gute Trennschärfen auf $\left(r_{\mathrm{i}(t-\mathrm{i})}>=0.6\right)$. Der Skala kann mit einem Cronbachs Alpha von $\alpha=$. 92 eine hohe Reliabilität zugesprochen werden. Zur Diagnosestellung werden alle Items aufsummiert: Personen mit Summenwerten größer als 41 werden als computerspielabhängig, Personen mit Summenwerten zwischen 35 und 41 als computerspielabhängigkeitsgefährdet eingestuft. Personen gefährdet im Sinne einer Abhängigkeitsproblematik lehnen somit die Items der Computerspielabhängigkeitsskala im Mittel nicht mehr ab. Dennoch kann hier noch nicht von einer Abhängigkeit ausgegangen werden, da Personen dieser Gruppe rein rechnerisch nicht allen Items in der Tendenz zustimmen und damit nicht alle Kernkriterien erfüllen können. ${ }^{164}$

Im englischen Sprachraum wurde ein Diagnoseinstrument zur Computerspielabhängigkeit für Kinder und Jugendliche von Gentile (Gentile et al., 2011; Gentile, 2009; Choo et al., 2010) entwickelt. Die Skala zur Erfassung pathologischer Computerspielnutzung (Pathological Video Game Use) orientiert sich in ihrer ursprünglichen Form an den Kriterien des pathologischen Spielens nach DSM-IV-TR ( $\mathrm{Saß}$ et al., 2003) indem diese in 11 Items eins zu eins auf den Gegenstandsbereich der Computerspielabhängigkeit umformuliert wurden. Insbesondere in der Erfassung der negativen Auswirkungen wurde dabei auf den schulischen Kontext der Kinder und Jugendlichen fokussiert. In der Überarbeitung

164 Mit den vergleichsweise strengen Cut-off-Werten soll dem sensiblen Gegenstand Rechnung getragen werden, indem zugunsten einer höheren diagnostischen Spezifität eine geringere diagnostische Sensitivität des Verfahrens in Kauf genommen wird (Rehbein et al., 2009a, 2009b, 2010). Eine Validierung des Instrumentes an klinischen Stichproben steht noch aus (Rehbein et al., 2009a, 2009b, 2010). 
der Skala (Gentile et al., 2011; Choo et al., 2010) wurde das Item zu den häuslichen Pflichten ("Do you sometimes skip hosehold chores in order to spend more time playing video games?") nicht mehr mit aufgennommen. Items eins, zwei, fünf und zehn wurden umformuliert. Mit den Items sechs ("Have you stolen a VG from a store or a friend, or stolen money in order to buy a VG?") und zehn (“" Have you ever needed to borrow money so you could get or play computer- or video-games?") werden erstmals auch die finanziellen Folgen pathologischen Glücksspielens mit auf den Gegenstansbereich des Computerspielens übertragen. Zusätzlich wurde in der Überarbeitung der Skala auch das zeitliche Kriterium des vergangenen Jahres mit eingeführt (vgl. Tabelle 66). Bei der Diagnosestel-

Tabelle 65. Computerspielabhängigkeitsskala (KFN-CSAS-II) von Rehbein, Mößle und Kleimann (Rehbein et al., 2009a, 2009b, 2010).

stimmt nicht (1), stimmt kaum (2), stimmt eher (3), stimmt genau (4)

\section{Einengung des Denkens und Verhaltens}

1. Ich beschäftige mich auch während der Zeit, in der ich nicht Computer- und Videospiele, gedanklich sehr viel mit Spielen.

2. Meine Gedanken kreisen ständig ums Computer- und Videospielen, auch wenn ich gar nicht spiele.

3. Zu bestimmten Zeiten oder in bestimmten Situationen spiele ich eigentlich immer: Das ist fast zu einer Routine für mich geworden.

4. Es kommt vor, dass ich eigentlich etwas ganz anderes tue und dann ohne zu überlegen ein Computerspiel starte.

\section{Negative Konsequenzen}

5. Meine Leistungen in der Schule leiden unter meinen Spielgewohnheiten.

6. Ich bin so häufig und intensiv mit Computer- und Videospielen beschäftigt, dass ich manchmal Probleme in der Schule bekomme.

7. Mir wichtige Menschen beschweren sich, dass ich zu viel Zeit mit Spielen verbringe.

8. Weil ich so viel spiele, unternehme ich weniger mit anderen.

\section{Kontrollverlust}

9. Ich verbringe oft mehr Zeit mit Computer- und Videospielen, als ich mir vorgenommen habe.

10. Ich habe das Gefühl, meine Spielzeit nicht kontrollieren zu können.

\section{Entzugserscheinungen}

11. Wenn ich nicht spielen kann, bin ich gereizt und unzufrieden.

12. Wenn ich längere Zeit nicht spiele, werde ich unruhig und nervös.

\section{Toleranzentwicklung}

13. Ich habe das Gefühl, dass Video- und Computerspiele für mich immer wichtiger werden.

14. Ich muss immer länger spielen, um zufrieden zu sein.

Computerspielabhängigkeitsgefährdet: Summenwert 35 - 41; computerspielabhän-

gig: Summenwert $>42$ 
lung wird analog zu den Kriterien des pathologischen Spielens nach DSM-IVTR (Saß et al., 2003) vorgegangen: Personen, die fünf oder mehr Symptomen zustimmen werden als computerspielabhängig klassifiziert; die Mittelkategorie wird dabei als halbe Zustimmung gewertet. Der Skala wird insgesamt eine hohe Konstruktvalidität zugesprochen (Choo et al., 2010).

Tabelle 66. Pathological Video Game Use nach Gentile (Gentile et al., 2011; Choo et al., 2010).

In the past year (yes (1), no (0), sometimes (.5)),...

1. Has your schoolwork suffered because you spent too much time playing computer- or video-games?

2. Have you ever skipped your studies or co-curricular activities to play more computeror video-games?

3. Do you need to spend more and more time and/or money on VGs to feel the same amount of excitement?

4. Have you played VGs to escape from problems, bad feelings, or stress?

5. Are you thinking about computer- or video-games more and more?

6. Have you stolen a VG from a store or a friend, or stolen money in order to buy a VG?

7. Have you tried to play VGs less often or for shorter periods of time, but are unsuccessful?

8. Have you become restless or irritable when trying to cut down or stop playing computer- or video games?

9. Have you ever lied to family or friends about how much you play VGs?

10. Have you ever needed to borrow money so you could get or play computer- or videogames?

computerspielabhängig: 5 oder mehr Symptomen muss zugestimmt werden, wobei eine Antwort mit sometimes als eine halbe Zustimmung gewertet wird.

In Rückgriff auf den Stand der Diagnostik von Internet- und Computerspielabhängigkeit kann damit zusammenfassend festgehalten werden, dass zunächst ein deutlicher Konsens unter den Studien dahin gehend feststellbar ist, dass für die Diagnosestellung auf Kriterien einer Substanzabhängigkeit nach DSM-IV-TR (Saß et al., 2003) oder des Abhängigkeitssyndroms nach ICD-10 (Dilling et al., 2006) zurückgegriffen werden muss (vgl. Tabelle 67). Mit Ausnahme der beiden Diagnoseinstrumente zur Internetabhängigkeit, ISS, in der die Dimension Einengung des Denkens und Verhaltens nicht enthalten ist, und CIUS, in der die Dimension Toleranzentwicklung nicht enhalten ist, finden sich in allen anderen Konzeptionen folgende fünf Kernkriterien: Einengung des (Denkens und) Verhaltens, Toleranzentwicklung, Kontrollverlust, Entzugssymptome und Fortsetzung trotz schädlicher Folgen. Hinsichtlich der Operationalisierung dieser Kriterien sowie der Cut-off-Werte, welche die Klassifikation einer Abhängigkeit begründen, zeigen sich jedoch große Unterschiede. Zusätzlich wird in manchen Operationalisierungen (DC-IA-A, OSVe-S und CSV-S) beispielsweise die reine mit Computerspielen verbrachte Zeit ab einer bestimmen Dauer als ein Abhän- 
gigkeitskriterium definiert. Eine Abgrenzung zwischen einem leidenschaftlichem, aber ansonsten unproblematischem Spielverhalten auf der einen Seite und einem suchtartigem Spielverhalten auf der anderen Seite wird hierdurch deutlich erschwert. So kann zeitlich exzessive Mediennutzung, wie sie unter Kindern und Jugendlichen häufig zu beobachten ist, zwar erstes Anzeichen einer tiefer gehenden Störung sein, ist aber letztlich kein Kernkriterium einer Abhängigkeitsproblematik (vgl. Rehbein et al., 2009b). Teilweise wurden aber auch Kriterien des Abhängigkeitssyndroms nach ICD-10 (Dilling et al., 2006) oder des pathologischen Spielens nach DSM-IV-TR (Saß et al., 2003) ohne eine Gewichtung für den Forschungsgegenstand unverändert auf die Computerspielnutzung übertragen. „Starkes Verlangen“ nach Ausübung der Nutzungstätigkeit, wie es in der CSVK-R, der CSV-S und der OSVe-S beinhaltet ist, ist zwar typisch für suchtartige Mediennutzung, aber aufgrund der hohen Attraktivität der Mediennutzung gerade für Kinder und Jugendliche - nur wenig geeignet, pathologische Mediennutzung von normaler Mediennutzung zu unterscheiden (vgl. Rehbein et al., 2009b). Ebenso scheinen die beiden Kriterien „Illegale Handlungen zur Finanzierung des Spiels“ und „Hoffnung auf Bereitstellung von Geld durch Dritte“ nur dann von Interesse zu sein, wenn mit dem Computerspielen auch ein Geldeinsatz verbunden ist. Dieser ist bei den klassischen Onlinerollenspielen durch die Fixkosten einer monatlichen Grundgebühr klar geregelt. Bei neueren Konzeptionen, in denen das Spiel über sog. Micropayments in Itemshops finanziert wird, könnten diese Kriterien jedoch trotz der gerade geübten Kritik an Bedeutung gewinnen.

Ein Merkmal, welches bis dato von den gängigen Messinstrumenten allerdings nur unzureichend erfasst wird, ist das zeitliche Bestehen negativer Auswirkungen, das lediglich in den Diagnosekriterien von Ko und Kollegen (DC-IA-A; Ko, C. H. et al., 2005) sowie im überarbeiteten Testinstrument von Gentile (Gentile et al., 2011; Choo et al., 2010) berücksichtigt wird. Nur so kann ausgeschlossen werden, dass kurzzeitige Episoden exzessiven Spielens, die durchaus auch kurzfristige Beeinträchtigungen mit sich bringen können, zu Unrecht als eine Internetabhängigkeit klassifiziert werden. In die gleiche Richtung weist die notwendige Berücksichtigung differenzialdiagnostischer Erwägungen. 
Tabelle 67. Diagnosekriterien einer Internet- oder Computerspielabhängigkeit.

\begin{tabular}{|c|c|c|c|c|c|c|c|c|c|c|}
\hline \multirow[b]{2}{*}{$\begin{array}{l}\text { Abhängigkeitssyn- } \\
\text { drom (ICD-10) } \\
\text { bzw. Substanzab- } \\
\text { hängigkeit (DSM- } \\
\text { IV) }\end{array}$} & \multirow[b]{2}{*}{$\begin{array}{l}\text { Pathologisches Spielen } \\
\text { (DSM-IV) }\end{array}$} & \multicolumn{2}{|c|}{$\begin{array}{c}\text { Diagnosti- } \\
\text { sche Krite- } \\
\text { rien Inter- } \\
\text { netabhän- } \\
\text { gigkeit }\end{array}$} & \multicolumn{7}{|c|}{ Testinstrumente } \\
\hline & & 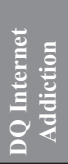 & 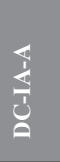 & $\mathscr{E}$ & $\stackrel{\infty}{\beta}$ & $\sum_{0}^{\infty}$ & $\sum_{0}^{\frac{\pi}{D}}$ & $\sum_{0}^{\infty}$ & $\sum_{\substack{\infty \\
0}}^{\infty}$ & $\sum_{2}^{2}$ \\
\hline $\begin{array}{c}\text { Einengung des } \\
\text { (Denkens und) Ver- } \\
\text { haltens }\end{array}$ & Starke Eingenommenheit & $\mathrm{x}$ & $\mathrm{x}$ & & $\mathrm{x}$ & $\mathrm{x}$ & $\mathrm{x}$ & $\mathrm{x}$ & $\mathrm{x}$ & $\mathrm{x}$ \\
\hline $\begin{array}{l}\text { Toleranzentwick- } \\
\text { lung }\end{array}$ & Steigerung der Einsätze & $\mathrm{x}$ & $\mathrm{x}$ & $\mathrm{x}$ & & $\mathrm{x}$ & $\mathrm{x}$ & $\mathrm{x}$ & $\mathrm{x}$ & $\mathrm{x}$ \\
\hline \multirow[t]{2}{*}{ Kontrollverlust } & $\begin{array}{l}\text { Wiederholte erfolglose Ver- } \\
\text { suche das Spiel zu kontrol- } \\
\text { lieren }\end{array}$ & $\mathrm{x}$ & $\mathrm{x}$ & $\mathrm{x}$ & $\mathrm{x}$ & $\mathrm{x}$ & $\mathrm{x}$ & $\mathrm{x}$ & $\mathrm{x}$ & $\mathrm{x}$ \\
\hline & $\begin{array}{l}\text { Wiederaufnahme nach Ver- } \\
\text { lusten }\end{array}$ & $\mathrm{x}$ & $\mathrm{x}$ & & & $\mathrm{x}$ & & $\mathrm{x}$ & $\mathrm{x}$ & \\
\hline Entzugssymptome & $\begin{array}{l}\text { Unruhe und Gereiztheit } \\
\text { beim Versuch, das Spiel ein- } \\
\text { zuschränken oder aufzuge- } \\
\text { ben }\end{array}$ & $\mathrm{x}$ & $\mathrm{x}$ & $\mathrm{x}$ & $\mathrm{x}$ & $\mathrm{x}$ & $\mathrm{x}$ & $\mathrm{X}$ & $\mathrm{x}$ & $\mathrm{x}$ \\
\hline $\begin{array}{l}\text { Fortsetzung trotz } \\
\text { schädlicher Folgen }\end{array}$ & $\begin{array}{l}\text { Gefährdung oder Verlust } \\
\text { wichtiger Beziehungen, von } \\
\text { Arbeitsplatz und Zukunfts- } \\
\text { chancen }\end{array}$ & $\mathrm{x}$ & $\mathrm{x}$ & $\mathrm{x}$ & $\mathrm{x}$ & $\mathrm{x}$ & $\mathrm{x}$ & $\mathrm{x}$ & $\mathrm{x}$ & $\mathrm{x}$ \\
\hline $\begin{array}{l}\text { Starkes Verlangen } \\
\quad \text { (nur ICD-10) }\end{array}$ & & & & & & $\mathrm{x}$ & $\mathrm{x}$ & $\mathrm{x}$ & & \\
\hline \multirow[t]{5}{*}{$\begin{array}{l}\text { Hoher Zeitaufwand } \\
\text { für Beschaffung, } \\
\text { Gebrauch und Erho- } \\
\text { lung }\end{array}$} & & & $\mathrm{x}$ & & & $\mathrm{x}$ & & $\mathrm{X}$ & & \\
\hline & $\begin{array}{l}\text { Spielen, um Problemen oder } \\
\text { negativen Stimmungen zu } \\
\text { entkommen }\end{array}$ & $\mathrm{x}$ & & & $\mathrm{x}$ & $\mathrm{x}$ & & $\mathrm{x}$ & & $\mathrm{x}$ \\
\hline & $\begin{array}{l}\text { Lügen gegenüber Dritten, } \\
\text { um das Ausmaß der Spiel- } \\
\text { problematik zu vertuschen }\end{array}$ & $\mathrm{x}$ & & & & & & & & $\mathrm{x}$ \\
\hline & $\begin{array}{l}\text { Illegale Handlungen zur Fi- } \\
\text { nanzierung des Spiels }\end{array}$ & & & & & & & & & $\mathrm{x}$ \\
\hline & $\begin{array}{l}\text { Hoffnung auf Bereitstellung } \\
\text { von Geld durch Dritte }\end{array}$ & & & & & & & & & $\mathrm{x}$ \\
\hline & Auswahlkriterium & $\begin{array}{c}5 \\
\text { von } \\
8\end{array}$ & $\begin{array}{c}7 \\
\text { von } \\
12\end{array}$ & $\begin{array}{l}\text { Cut- } \\
\text { off }\end{array}$ & & $\begin{array}{l}\text { Cut- } \\
\text { off }\end{array}$ & $\begin{array}{c}3 \\
\text { von } \\
6\end{array}$ & $\begin{array}{l}\text { Cut- } \\
\text { off }\end{array}$ & $\begin{array}{l}\text { Cut- } \\
\text { off }\end{array}$ & $\begin{array}{c}6 \\
\text { von } \\
11\end{array}$ \\
\hline & Zeitkriterium & & $\mathrm{x}$ & & & & & & & $\mathrm{x}$ \\
\hline
\end{tabular}




\subsubsection{Komorbidität und Risikokorrelate einer Internet- und}

Computerspielabhängigkeit

Zur Charakterisierung und Etablierung eines Krankheitsbildes Internet- oder Computerspielabhängigkeit sowie zu dessen Differenzierung gegenüber bekannten Störungsbildern ist eine Betrachtung möglicher komorbider Erkrankungen unerlässlich. Daten hierzu kommen aus zwei unterschiedlichen Quellen, einer psychiatrischen Diagnostik in der klinisch-psychiatrischen Praxis sowie anonymen Datenerhebungen (z. T. im Internet) mittels psychometrischer (Selbst-)beurteilungsbögen. Neben der fehlenden eindeutigen Regelung der Diagnosestellung des zu untersuchenden Konstrukts Internet- oder Computerspielabhängigkeit in beiden Forschungsbereichen ist dies in methodischer Hinsicht doppelt problematisch: In der klinisch-psychiatrischen Praxis wird zwar eine klinische Diagnose, z. B. Depression, gestellt. Dies erfolgt aber immer nur für eine geringe Fallzahl an Patienten, für die zudem durch den Kontext, in dem sie vorstellig werden, auch besonders schwere psychische Belastungen zu erwarten sind. In Studien mit psychometrischen Selbstbeurteilungsbögen wird auf der anderen Seite durchwegs eine größere Anzahl von Personen befragt, eine zwingend notwendige klinische Diagnose erfolgt hier jedoch nicht. Zusätzlich zu möglichen Verfälschungen, beispielsweise durch die Anonymität der Befragungen im Internet, verbleiben die Daten in diesem Bereich somit auf einem subklinischen Niveau. Die Ergebnisse sind somit jeweils mit der gebotenen Vorsicht zu beurteilen.

Komorbidität. Die bisherige Studienlage zu in der klinischen Praxis vorstelligen Internet- oder Computerspielabhängigen deutet darauf hin, dass ein beträchtlicher Anteil derjenigen, die mit den bisher zur Verfügung stehenden Mitteln als abhängig identifiziert werden, die Kriterien für eine weitere bekannte psychische Störung erfüllen, wobei depressive Störungen, Angsterkrankungen, Aufmerksamkeitsdefizit- und Hyperaktivitätsstörung (ADHS) sowie Persönlichkeitsstörungen besonders oft auftreten: Am Häufigsten (33-78\%) lassen sich bei Internet- oder Computerspielabhängigen unspezifische depressive Syndrome feststellen (vgl. Black \& Moyer, 1998; Greenfield, 2000; te Wildt et al., 2007). Etwas weniger häufig werden Angsterkrankungen (18-50\%) diagnostiziert (vgl. Shapira, Goldsmith, Keck, Khosla \& McElroy, 2000; Kratzer \& Hegerl, 2007), insbesondere soziophobe Störungen (vgl. Campbell, Cumming \& Hughes, 2006). Zudem konnte in verschiedenen Studien ein Zusammenhang zwischen pathologischer Mediennutzung und ADHS (24-58\%) gezeigt werden (vgl. Yoo et al., 2004; Yen, J. Y. et al., 2008; Ha et al., 2006; Ko, C. H., Yen, Chen, Chen \& Yen, 2008; Chan \& Rabinowitz, 2006). Persönlichkeitsstörungen, die als schwerwiegende Bindungsstörungen nicht selten zusätzlich zu einer Achse-I-Störung festzustellen sind, werden ebenfalls im Zusammenhang mit Internetabhängigkeit 
beschrieben (vgl. Black \& Moyer, 1998), wobei es hier noch an aussagekräftigen Studienergebnissen fehlt. Die Art der in der klinisch-psychiatrischen Praxis ermittelten Komorbiditäten von Internet- und Computerspielabhängigen entspricht somit weitgehend denen von Menschen mit stoffgebundenen Abhängigkeitserkrankungen, insbesondere denen von Alkoholabhängigen (vgl. Driessen, 1999). In der deutschen Gesamtbevölkerung finden sich für Erwachsene zum Vergleich im Mittel die folgenden 12-Monats-Prävalenzraten: depressive Störungen 8.3\%, Angsterkrankungen $14.5 \%$, soziale Phobie 2.0\% (Wittchen \& Hoyer, 2006). Für Kinder und Jugendliche haben die häufigsten Störungen die folgenden Prävalenzraten: Angststörungen 10.4\%, dissoziative Störungen 7.5\%, depressive Störungen $4.4 \%$, hyperkinetische Störungen $4.4 \%$ (Ihle \& Esser, 2002).

Zur Beurteilung der psychischen Belastung wird in Studien mit psychometrischen Selbstbeurteilungsbögen als ökonomisches psychodiagnostisches Verfahren zumeist die Symptom-Checkliste (SCL-90-R; Derogatis, 1977; Franke, 2002) oder deren Kurzform, das Brief-Symptom-Inventory (BSI; Franke, 2000), verwendet. So konnten Yang und Kollegen (Yang, Choe, Baity, Lee \& Cho, 2005) in einer Studie an 328 koreanischen Jugendlichen zwischen 15 und 19 Jahren eine erhöhte Symptombelastung der abhängigen Internetnutzer (4.9\%) im SCL-90$\mathrm{R}$ auf den Subskalen Somatisierung, Zwanghaftigkeit, Unsicherheit im Sozialkontakt, Depressivität, Ängstlichkeit, Aggressivität/Feindseligkeit, phobische Angst und Psychotizismus identifizieren (allerdings nur im Vergleich zu den Wenignutzern statistisch bedeutsam). Zu ähnlichen Ergebnissen kommen auch Mößle und Rehbein (2008) in einer Befragung von 3.278 im Mittel 19-jährigen jungen Erwachsenen. Neben einem erhöhten Leidensdruck abhängiger Spieler (diagnostiziert mit der KFN-CSAS-I) in verschiedenen Bereichen ihres Lebens (Partnerschaft, Familienleben, Sozialkontakte, Freizeitgestaltung, allgemeines Wohlbefinden, Gesundheit, Leistungsfähigkeit, Ausbildung/Arbeit, Finanzielle Situation), zeigten sich im $B S I$ deutliche Auffälligkeiten unter abhängigen Computerspielern, auch im Vergleich zu den Exzessivspielern. Bezüglich aller neun erfassten Lebensbereiche berichteten abhängige Computerspieler von deutlichen Beeinträchtigungen, während sich hier nicht-abhängige Exzessivspieler gar nicht oder nur geringfügig von Normalspielern unterschieden. Zusätzlich fanden sich im BSI gegenüber der Gruppe der Viel- und Exzessivspieler, von der insgesamt 14 Prozent eine auffällige psychische Belastung auf mindestens einer der im BSI erhobenen Dimensionen aufwies, unter den nach KFN-CSAS-I abhängigen Spielern 49 Prozent mit einer auffälligen psychischen Belastung (Mößle \& Rehbein, 2008). Während sich Viel- und Exzessivspieler auf keiner der Unterdimensionen des BSI signifikant von normal-spielenden Befragten unterschieden, ergaben sich bei abhängigen Spielern signifikant erhöhte Belastungswerte auf allen Unterdimensionen (Mößle \& Rehbein, 2008). 
Kritisch anzumerken zu beiden Forschungsbereichen ist, dass aufgrund des querschnittlichen Untersuchungsdesigns der bisherigen Studien zu komorbiden Erkrankungen einer Internet- oder Computerspielabhängigkeit, grundsätzlich offen bleibt, ob diese nur gemeinsam auftreten oder eine Erkrankung die andere bedingt. In einer ersten zweijährigen Längsschnittstudie mit 2.998 Schülerinnen und Schülern aus Singapur konnten Gentile und Kollegen (Gentile et al., 2011) Depressionen, gemessen mit der Asian Adolescent Depression Scale (Woo et al., 2004), Angst, gemessen mit The Screen for Child Anxiety-Related Emotional Disorders (SCARED; Birmaher et al., 1997), und Soziale Phobien, gemessen mit dem Social Phobia Inventory (SPIN; Connor et al., 2000) erstmalig als Folge einer pathologischen Computerspielnutzung nachweisen: Jugendliche, die im Erhebungszeitraum zu pathologischen Computerspielern wurden, hatten auf allen drei Skalen in Folge höhere Werte; Jugendliche, die im Erhebungszeitraum nicht mehr als pathologische Computerspieler klassifiziert wurden, hatten auf allen drei Skalen in Folge geringere Werte als jene Jugendlichen, die weiterhin als computerspielabhängig klassifiziert wurden. Die Autoren interpretieren ihre Befunde allerdings trotz dieser einseitigen kausalen Wirkrichtung eher im Sinne einer Wechselwirkung: depressiv verstimmte Jugendliche wenden sich vermehrt dem Computerspiel zu und werden dadurch wiederum depressiver. Um dies zu überprüfen wären aber länger angelegte Längsschnittstudien notwendig.

Über alle Studien zusammengenommen bleibt für Internet- oder Computerspielabhängige neben einem hohen Leidensdruck zumindest eine hohe psychische Belastung, beobachtet in psychometrischen Befragungsstudien, festzuhalten, welche sich auch in einer hohen Anzahl komorbider Störungen im klinischen Kontext manifestiert. Eine Aussage über den genauen Wirkzusammenhang ist zum jetzigen Zeitpunkt leider noch nicht möglich.

Risikokorrelate. Insbesondere in psychometrischen Befragungsstudien werden darüber hinaus neben den beobachteten komorbiden Störungen weitere Risikokorrelate einer Internet- oder Computerspielabhängigkeit betrachtet. So konnten als mediennutzungsspezifische Risikofaktoren einer Internet- oder Computerspielabhängigkeit in bisherigen Untersuchungen neben hohen Spielzeiten (vgl. Tejeiro Salguero \& Bersabe Morán, 2002; Gentile, 2009; Rehbein et al., 2009b, 2010; Choo et al., 2010; Wölfling et al., 2010b; Gentile et al., 2011) ein dysfunktionales medienbezogenes Coping (vgl. Batthyány, Müller, Benker \& Wölfling, 2009; Rehbein et al., 2010; Wölfling et al., 2010b) sowie insbesondere die Nutzung von Onlinerollenspielen (MMORPGs) (vgl. Rehbein et al., 2010; Wölfling et al., 2010b; Schmidt, J.-H., Drosselmeier, Rohde \& Fritz, 2011) identifiziert werden. Im Schulkontext waren dies schlechtere Schulleistungen (vgl. Anderson, C. A. \& Dill, 2000; Walsh, 2000; Rehbein et al., 2009b, 2010; Batthyány et al., 2009; Gentile, 2009; Choo et al., 2010; Gentile et al., 2011; Wölfling et al., 
2010b), ein erhöhter Schulabsentismus - insbesondere um zu Spielen - (vgl. Rehbein et al., 2009b, 2010; Choo et al., 2010; Wölfling et al., 2010b), das Wiederholen einer Klasse (vgl. Rehbein et al., 2009b, 2010) sowie eine selbst berichtete Schulangst (vgl. Batthyány et al., 2009; Rehbein et al., 2010; Wölfling et al., 2010b). Als gesundheitsbezogene Folgen einer suchtartigen Mediennutzung werden des Weiteren geringe Schlafenszeiten (Rehbein et al., 2009b, 2010; Choo et al., 2010) sowie andere nutzungsbezogene Gesundheitsprobleme, wie z. B. des Bewegungsapparates, berichtet (vgl. Cleary, McKendrick \& Sills, 2002; Griffiths, 2002, 2005; Gentile, 2009; Choo et al., 2010). Im Bereich der Persönlichkeit zeichnen sich die suchtartigen Nutzer zudem u. a. durch Aufmerksamkeitsprobleme/Impulsivität (vgl. Hahn \& Jerusalem, 2001a; Gentile, 2009; Rehbein et al., 2009b, 2010; Choo et al., 2010), eine erhöhte Gewaltakzeptanz (vgl. Rehbein et al., 2009b, 2010; Choo et al., 2010), mangelnde Fähigkeiten zur Perspektivenübernahme (vgl. Rehbein et al., 2010), soziale Unsicherheit/Schüchternheit (vgl. Wölfling et al., 2010b) oder allgemein geringere soziale Kompetenzen (vgl. Choo et al., 2010) aus. Darüber hinaus berichten computerspielabhängige Jugendliche über das Fehlen regelmäßiger Freizeitengagements (vgl. Rehbein et al., 2009b, 2010) und äußern häufiger Selbstmordgedanken (vgl. Rehbein et al., 2009b, 2010).

Um eine Aussage über den genauen Wirkzusammenhang (Ursache, Korrelat, Folge) sowie die Bedeutung der jeweiligen Risikokorrelate treffen zu können, wurden in einigen wenigen Studien die unterschiedlichen Risikokorrelate auch (gemeinsam) als erklärende Faktoren einer Computerspielabhängigkeit überprüft. Rehbein und Kollegen (Rehbein et al., 2010) realisierten dies zum Beispiel in einem logistischen Regressionsmodell, welches aufklären sollte, mit welchen personengebundenen, situativen und computerspielbezogenen Variablen sich die Entstehung einer Computerspielabhängigkeit möglichst gut vorhersagen lässt. Hierbei sollte auch überprüft werden, ob der Nutzung bestimmter Spielgenres noch ein Einfluss auf die Entstehung von Computerspielabhängigkeit zukommt, wenn weitere relevante Einflussgrößen in die Regression aufgenommen werden. Folgende Variablen erwiesen sich dabei als bedeutsame Prädiktoren einer Computerspielabhängigkeit: dysfunktionale Mediennutzung, Macht- und Kontrollerleben in Computerspielen, die Nutzung von MMORPGs, Erfolgserlebnisse vor allem in Computerspielen, Schulangst, Klassenwiederholungen, geringere Fähigkeit zur Perspektivenübernahme, Impulsivität mit negativen Handlungskonsequenzen und Gewaltakzeptanz (Rehbein et al., 2010). Darüber hinaus konnten Gentile und Kollegen (Gentile et al., 2011) in der bisher einzigen längsschnittlichen Betrachtung bestehender Risikokorrelate einer Computerspielabhängigkeit die Entwicklung einer Depression, Angst, soziale Phobien und geringere Schulleistungen als Folgen pathologischer Computerspielnutzung identifizieren, wo- 
hingegen eine hohe wöchentliche Nutzungszeit, eine geringe soziale Kompetenz, eine geringe Empathie sowie erhöhte Impulsivitätswerte in dieser Studie als Prädiktoren einer Computerspielabhängigkeit ausgemacht wurden.

\subsubsection{Epidemiologie von Internet- und Computerspielabhängigkeit}

Obwohl die genaue diagnostische Einordnung des Phänomens Internet- und Computerspielabhängigkeit (auch vor dem Hintergrund differenzialdiagnostischer Erwägungen) noch nicht geklärt ist (siehe oben), existieren zunehmend Forschungsbemühungen, diese näher zu untersuchen und den Anteil der davon betroffenen Personen in der gesamten Bevölkerung oder bestimmten Populationen zu bestimmen. Aufgrund der bereits dargestellten Heterogenität in der Erfassung des Phänomens fallen jedoch die bislang vorliegenden Prävalenzraten, d. h. die geschätzten Anteile an abhängigen Personen, sehr uneinheitlich aus. Zudem werden in den verschiedenen Untersuchungen Personengruppen untersucht, die in Alter und Mediennutzungsstatus z. T. deutlich voneinander abweichen. Eine „Pathologisierung“ des Computerspielens angesichts teilweise zweistelliger Abhängigkeitsprävalenzen ist dabei verschiedentlich kritisiert worden (Griffiths, 2009; Rehbein et al., 2009b; Rehbein \& Borchers, 2009; Charlton \& Danforth, 2007). Studien, die Anspruch auf Repräsentativität erheben können, liegen bislang nur in begrenztem Ausmaß vor.

Im Folgenden sollen einige nationale wie internationale Studien, die eine Prävalenzschätzung ermöglichen, getrennt für die Bereiche Internet- (vgl. Tabelle 68) und Computerspielabhängigkeit (Tabelle 69) vorgestellt werden, wobei der Schwerpunkt auf den Studien zur Computerspielabhängigkeit liegen soll. Auswahlkriterium war die Berücksichtigung einer jugendlichen und/oder kindlichen Stichprobe. 
Tabelle 68. Prävalenz von Internetabhängigkeit in unterschiedlichen Altersgruppen.

\begin{tabular}{|c|c|c|c|c|c|}
\hline $\begin{array}{c}\text { Autoren } \\
\text { (Jahr) } \\
\text { Land }\end{array}$ & $\begin{array}{l}\text { Methode und } \\
\text { Altersgruppe }\end{array}$ & $\begin{array}{l}\text { Anzahl Befrag- } \\
\text { te }(\mathrm{N}) \text {, Durch- } \\
\text { schnittsalter in } \\
\text { Jahren (M) }\end{array}$ & Anteil $\widehat{\partial}$ & $\begin{array}{l}\text { Anteil abhängige } \\
\text { bzw. gefährdete } \\
\text { Personen }\end{array}$ & $\begin{array}{l}\text { Operationalisie- } \\
\text { rung }\end{array}$ \\
\hline $\begin{array}{l}\text { Greenfield } \\
\text { (1999) } \\
\text { USA }\end{array}$ & $\begin{array}{l}\text { Onlinebefra- } \\
\text { gung } \\
\text { Altersspanne } \\
\quad 8-85\end{array}$ & $\begin{array}{c}\mathrm{N}=17.251 \\
M=33\end{array}$ & $71 \%$ & $5.7 \%$ & $\begin{array}{l}\text { Checkliste des Vir- } \\
\text { tual Addiction Sur- } \\
\text { vey (VAS). } \\
10 \text { dichotome } \\
\text { Items in Anleh- } \\
\text { nung an } \\
\text { Spielsucht nach } \\
\text { DSM-IV }\end{array}$ \\
\hline $\begin{array}{l}\text { Hahn \& } \\
\text { Jerusalem } \\
\text { (2001a) }^{165} \\
\text { Deutsch- } \\
\text { land }\end{array}$ & $\begin{array}{l}\text { Onlinebefra- } \\
\text { gung von In- } \\
\text { ternetnutzern }\end{array}$ & $\begin{array}{c}\mathrm{N}=8.859 \\
<15 \text { Jahren: } 107 \\
\text { 15 Jahre: } 164 \\
\text { 16 Jahre: } 288 \\
\text { 17 Jahre: } 333 \\
\text { 18 Jahre: } 331\end{array}$ & $\begin{array}{c}80 \% \\
<15: \\
78 \% \\
15: 77 \% \\
16: 84 \% \\
17: 87 \% \\
18: 90 \%\end{array}$ & $\begin{array}{c}\text { gf.: } 6.6 \% \text {; ah.: } 3.2 \% \\
<15 \text { - gf.: } 9.3 \% \text {, ah.: } \\
10.3 \% \\
15 \text { - gf.: } 14.6 \% \text {, ah.: } \\
9.1 \% \\
16 \text { - gf.: } 7.6 \% \text {, ah.: } \\
8.7 \% \\
17 \text { - gf.: } 9.9 \% \text {, ah.: } \\
5.7 \% \\
18 \text { - gf.: } 10.9 \% \text {, ah.: } \\
5.7 \%\end{array}$ & $\begin{array}{l}\text { Internetsuchtskala } \\
\quad(\text { ISS-20) }\end{array}$ \\
\hline $\begin{array}{l}\text { Lin \& Tsai } \\
\text { (2002) } \\
\text { Taiwan }\end{array}$ & $\begin{array}{c}\text { Schülerbefra- } \\
\text { gung } \\
\text { 10., } 11 ., 12 . \\
\text { Jahrgangsstu- } \\
\text { fe } \\
\text { Nur Internet- } \\
\text { nutzer }\end{array}$ & $\begin{aligned} \mathrm{N} & =753 \\
M & =11.8\end{aligned}$ & $67.5 \%$ & $11.8 \%$ & $\begin{array}{l}\text { Internet Addiction } \\
\text { Scale for Taiwa- } \\
\text { nese high school- } \\
\text { ers (IAST), } 29 \\
\text { Items. }\end{array}$ \\
\hline $\begin{array}{l}\text { Yang et al. } \\
(2005) \\
\text { Korea }\end{array}$ & $\begin{array}{l}\text { Schülerbefra- } \\
\text { gung } \\
\text { Altersspanne } \\
15-19\end{array}$ & $\begin{array}{l}\quad N=328 \\
\text { 10. }(15-17): 110 \\
\text { 11. }(16-18): 109 \\
\text { 12. }(17-19): 109\end{array}$ & $\begin{array}{l}10 .: 56 \% \\
11 .: 54 \% \\
12 .: 54 \%\end{array}$ & $4.9 \%($ davon $63 \%$ ठૈ) & $\begin{array}{c}\text { Internet Addiction } \\
\text { Test }\end{array}$ \\
\hline $\begin{array}{l}\text { Müller, } \\
\text { K.W. et al. } \\
\text { (2011) } \\
\text { Deutsch- } \\
\text { land }\end{array}$ & $\begin{array}{l}\text { Repräsenta- } \\
\text { tivbefragung } \\
\text { (Face-to- } \\
\text { Face) } \\
\text { Altersspanne } \\
14-94\end{array}$ & $\begin{array}{c}\mathrm{N}=2.512 \\
\mathrm{~N}=1.382 \\
\text { (Internetnutzer) } \\
<\text { 25 Jahren: } 229\end{array}$ & $49 \%$ & $\begin{array}{c}4.2 \% \\
<25: 5.2 \%\end{array}$ & $\begin{array}{l}\text { Assessment of In- } \\
\text { ternet and Comput- } \\
\text { er game Addiction } \\
\text { (AICA-S) }\end{array}$ \\
\hline
\end{tabular}

Anmerkung. Befragungen an spezifischen Spielerpopulationen (z. B. Spieler des Rollenspiels Everquest) wurden aufgrund ihrer hohen Selektivität in dieser Zusammenstellung nicht berücksichtigt.

165 Es werden in dieser Studie zwar auch nach Geschlecht getrennte Prävalenzwerte ausgegeben, die allerdings aufgrund der geringen Fallzahl in den unterschiedlichen Altersgruppen nicht berichtet werden. 
Prävalenz von Internetabhängigkeit. Eine US-amerikanische Studie von Greenfield (1999) mit 17.521 Versuchspersonen ergab bereits zu Ende des letzten Jahrtausends ein missbräuchliches Internetnutzungsverhalten, gemessen mit einer Checkliste des Virtual Addiction Survey, bei etwa 5.7 Prozent der Internetnutzer zwischen 8 und 85, wobei leider nicht nach Altersgruppen unterschieden wurde. Für Deutschland hat eine Onlinestudie von Hahn und Jerusalem (2001b, 2001c, 2001a) mit 8.859 Versuchspersonen zu einer vergleichbaren Zeit mithilfe der Internetsuchtskala (ISS) geringere Abhängigkeitsraten bei den Internetnutzern ermittelt (3.2\%, gefährdet: $6.6 \%$ ). Dieser geringe Prävalenzwert ist jedoch hier vor allem durch die älteren Altersgruppen (über 20 Jahren) der Befragung bedingt; bei den Unter- 15-jährigen lagen die Prävalenzraten mit 9.3 Prozent gefährdeten und 10.3 Prozent süchtigen Internetnutzern deutlich höher. So fanden auch Lin und Tsai (2002) für eine ,junge“ Untersuchungsstichprobe 753 taiwanesischer Highschoolschüler mit einem Durchschnittsalter von 12 Jahren mit 11.8 Prozent eine deutlich höhere Rate an Abhängigen. Yang und Kollegen (Yang et al., 2005) berichten schließlich in einer Studie an 328 koreanischen Jugendlichen zwischen 15 und 19 Jahren von 4.9 Prozent abhängigen Internetnutzern, zwei Drittel davon männlichen Geschlechts. Allerdings sind all diese Zahlen aus nunmehr bereits mehrere Jahre zurückliegenden Untersuchungen mit Vorsicht zu betrachten, da sich die Nutzung neuer elektronischer Medien insbesondere bei den Heranwachsenden deutlich verändert hat. So fallen in einer aktuellen nationalen Studie von Müller, K.W. und Kollegen (2011) in einer ersten Repräsentativbefragung zur Internetabhängigkeit mit 2.512 Versuchspersonen im Alter von 14 bis 94 Jahren, die Prävalenzwerte einer Internetabhängigkeit im Vergleich zu Hahn und Jerusalem (2001b, 2001c, 2001a) mit 4.2 Prozent etwas höher aus; und dies bei einem deutlich geringeren Anteil an Männern in der Stichprobe (49\% im Vergleich zu 80\%).

Prävalenz von Computerspielabhängigkeit. Auch für Computerspielabhängigkeit ergibt sich bislang eine deutliche Schwankungsbreite zwischen den vorliegenden Prävalenzschätzungen (Tabelle 69). So untersuchte Yang (2001) eine Stichprobe von fast 1.300 koreanischen Schülern unterschiedlicher Jahrgangsstufen mit einem Durchschnittsalter von etwa 15 Jahren. Der Autor konzentrierte sich global auf Abhängigkeiten in Zusammenhang mit Computernutzung, Computerspielen war in der Stichprobe jedoch die relevanteste Computeraktivität. Anhand einer 20 Items umfassenden Skala, die jedoch nicht genau dokumentiert wird, gelangte er zu einem Anteil von 6.1 Prozent pathologischer Computernutzer. Hauge und Gentile (2003) kommen in einer ähnlichen Altersgruppe von über 600 US-amerikanischen Schülern (8. und 9. Jahrgangsstufe) zu einem mehr als doppelt so hohen Anteil an computerspielabhängigen Jugendlichen (15\%). 
Die Erfassung der Abhängigkeit erfolgte in dieser Studie durch sieben Items, die jedoch ebenfalls nicht näher beschrieben werden.

Grüsser und Kollegen (2005) berichten in einer ersten Erhebung für eine Stichprobe von 323 deutschen Sechstklässlern über 9.3 Prozent computerspielsüchtige Kinder, die mit Hilfe des CSVK klassifiziert wurden. In einer späteren Untersuchung derselben Forschergruppe (Wölfling et al., 2008) mit 221 deutschen Achtklässlern sinken die Prävalenzzahlen für computerspielsüchtige Kinder, klassifiziert mit dem CSVK-R, auf 6.3 Prozent. In einer weiteren Studie mit 642 Kindern und Jugendlichen aus Nordrhein-Westfalen und 1.068 Kindern und Jugendlichen aus Wien, jeweils zwischen 13 und 18 Jahren, werden schließlich 12.3 Prozent der Wiener und 8.4 Prozent der nordrhein-westfälischen Kinder und Jugendlichen als computerspielsüchtig eingeordnet (Wölfling et al., 2010b). Zur Diagnose wurde hier der CSV-S verwendet.

Die beiden stichprobenbezogen größten Studien, die sich weltweit mit der Erforschung der Verbreitung und der Ursachen von Computerspielabhängigkeit beschäftigt haben, waren bislang die KFN-Schülerbefragung 2005 sowie die KFNSchülerbefragung 2007/2008, die entweder für spezifische Regionen Deutschlands oder für Gesamtdeutschland Repräsentativität für die befragten Neuntklässler beanspruchen können (Mößle et al., 2007; Baier \& Rehbein, 2009; Rehbein et al., 2009b, 2010). Innerhalb der KFN-Schülerbefragung 2005 mit 14.301 Schülerinnen und Schülern wurden anhand der KFN-CSAS-I 5.1 Prozent der befragten Neuntklässler als computerspielabhängig oder abhängigkeitsgefährdet eingestuft, wobei sich zeigte, dass fast ausschließlich männliche Befragte ein pathologisches Spielverhalten aufwiesen. Bei exklusiver Betrachtung männlicher Jugendlicher ergab sich eine Prävalenzrate von 8.9 Prozent abhängiger oder gefährdeter Spieler. Innerhalb der KFN-Schülerbefragung 2007/2008 mit 15.168 Schülerinnen und Schüler wurde anhand der KFN-CSAS-II mit 4.5 Prozent ein etwas geringerer Anteil der befragten Neuntklässler als computerspielabhängig oder abhängigkeitsgefährdet eingestuft. Auch hier zeigten fast ausschließlich männliche Befragte ein pathologisches Spielverhalten: 7.7 Prozent abhängige oder gefährdete Spieler. In einer weiteren Befragung junger Erwachsener der Autoren, in der insgesamt 2.667 Berufsschüler aus Osnabrück zu ihrem Computerspielverhalten befragt wurden, kommen diese zu etwas geringeren Prävalenzzahlen für die ältere Stichprobe (Rehbein et al., 2011): Hier wurden 0.9 Prozent der 16-18-jährigen Schüler und 0.6 Prozent der über 18-Jährigen als abhängig sowie $1.7 \%$ der 16-18-jährigen Schüler und 0.7 Prozent der über-18-Jährigen als gefährdet eingestuft. 
Schmidt, J.-H. und Kollegen (2011) adaptierten zudem die KFN-CSAS-II ${ }^{166}$ für eine deutschlandweite repräsentative Telefonbefragung mit 600 Versuchspersonen. „Zielgruppe für die Untersuchung waren Personen im Alter ab 14 Jahren in Haushalten mit Telefonfestnetzanschluss in Deutschland, die Handy-, Computer- oder Konsolenspiele [...] zumindest 'selten' spielen." (Schmidt, J.-H. et al., 2011, S. 38). ${ }^{167}$ Für diese altersheterogene Population an Computerspielern kommen die Autoren zu vergleichbaren Ergebnissen: 0.5 Prozent der Versuchspersonen wurden als abhängig sowie $0.9 \%$ als gefährdet eingestuft. Der Anteil suchtartig spielender Personen liegt unter den Jugendlichen zwischen 14 und 19 Jahren jedoch deutlich höher, bei 3.5 Prozent Gefährdeten und 0.9 Prozent Abhängigen. Aufgrund der geringen Fallzahl $(n=114)$ sind die Ergebnisse jedoch mit gegebener Vorsicht zu betrachten.

Gentile (2009) berichtet in einer für die USA repräsentativen Onlinebefragung mit 8 bis 18 Jahre alten Kindern und Jugendlichen wiederum einen etwas höheren Prozentsatz an abhängigen Computerspielern: 11.9 Prozent der Jungen und 2.9 Prozent der Mädchen wurden in dieser Studie anhand einer 11 Items umfassenden Abhängigkeitsskala (basierend auf den DSM-IV-Kriterien für pathologisches Spielen) als abhängig klassifiziert. In einer zweijährigen Längsschnittstudie mit 2.998 Schülerinnen und Schülern zweier unterschiedlicher Altersgruppen aus Singapur, berichten Gentile und Kollegen (Gentile et al., 2011) zudem über alle drei Messzeitpunkte gemittelt eine Prävalenzrate von 9.9 Prozent der Jugendlichen (Jungen: 12\%, Mädchen: 4.6\%), die als computerspielabhängig eingestuft werden müssen. Klassifiziert wurde analog zu Gentile (2009). In dieser Studie jedoch mit einer um ein Item verkürzten 10 Items umfassenden Abhängigkeitsskala. Während der zweijährigen Befragungsdauer wurde ferner lediglich ein weiteres Prozent der Jugendlichen zusätzlich als computerspielabhängig eingestuft. 84 Prozent der zu Messzeitpunkt eins als abhängig klassifizierten Jugendlichen, waren dies auch noch nach zwei Jahren. Pathologisches Computerspielen ist dementsprechend nicht nur ein passageres Jugendphänomen, sondern ein länger andauerndes Störungsbild.

Damit bewegen sich die bislang vorliegenden Prävalenzschätzungen von Internet- und Computerspielabhängigkeit bzw. Abhängigkeitsgefährdung je nach untersuchter Population und Stichprobenzugang zwischen 3 und 15 Prozent und weisen damit nach wie vor eine hohe Spannweite auf. Schlussfolgerungen zum

166 Neben der Durchführung als mündliche Telefoninterviews ohne Vorlage einer Printversion, wurde die Instruktion abgeändert, die Itemsaklierung umgepolt und 7 von 14 Items leicht in ihrem Wortlaut verändert (Geändert wurde die Phrase "Computer- und Videospiele\&quot; in "solche Spiele\&quot; und die Phrase "in der Schule\&quot; in "in der Schule, in der Ausbildung, im Studium oder im Beruf\&quot;; vgl. Fritz, Lampert, Schmidt \& Witting, 2011).

167 Eine Rücklaufstatistik wird leider nicht berichtet. 
Verlauf der Internet- und Computerspielabhängigkeit über verschiedene Altersgruppen, sind, da nur selten nach Altersgruppen differenzierte Auswertungen berichtet werden und zudem unterschiedliche Messinstrumente zum Einsatz kamen, leider nicht möglich. Durchgängig zeigt sich jedoch in allen Forschungen, dass Jungen stärker betroffen sind als Mädchen.

Tabelle 69. Prävalenz von Computerspielabhängigkeit in unterschiedlichen Altersgruppen.

\begin{tabular}{|c|c|c|c|c|c|}
\hline $\begin{array}{l}\text { Autoren } \\
\text { (Jahr) } \\
\text { Land }\end{array}$ & $\begin{array}{l}\text { Methode und } \\
\text { Altersgruppe }\end{array}$ & $\begin{array}{l}\text { Anzahl Befrag- } \\
\text { te (N), Durch- } \\
\text { schnittsalter in } \\
\text { Jahren (M) }\end{array}$ & Anteil $\bar{\partial}$ & $\begin{array}{l}\text { Anteil abhängige } \\
\text { bzw. gefährdete } \\
\text { Personen }\end{array}$ & $\begin{array}{l}\text { Operationalisie- } \\
\text { rung }\end{array}$ \\
\hline $\begin{array}{l}\text { Yang } \\
(2001) \\
\text { Korea }\end{array}$ & $\begin{array}{l}\text { Schülerbefra- } \\
\text { gung } \\
\text { 7. - 12. Jahr- } \\
\text { gangsstufe }\end{array}$ & $\begin{array}{c}\mathrm{N}=1.296 \\
M=14.8 \\
\text { 7. }(12-13): 212 \\
\text { 8. }(13-14): 221 \\
\text { 9. }(14-16): 226 \\
\begin{array}{c}10 .(15-17): \\
211\end{array} \\
\text { 11. }(16-18): \\
212 \\
\text { 12. }(17-19): \\
214\end{array}$ & $\begin{array}{c}51 \% \\
7 .: 52 \% \\
8 .: 52 \% \\
9 .: 50 \% \\
10 .: 51 \% \\
11 .: 50 \% \\
12 .: 49 \%\end{array}$ & $\begin{array}{c}6.1 \% \\
\text { (allgemeine Compu- } \\
\text { terabhängigkeit) } \\
\text { Keine Differenzierung } \\
\text { nach Altersgruppen }\end{array}$ & $\begin{array}{l}\text { Computer-related } \\
\text { addictive behavior } \\
\text { inventory (CRA- } \\
\text { BI), } 20 \text { Items adap- } \\
\text { tiert von Internet- } \\
\text { suchtmessung von } \\
\text { Young (1998) }\end{array}$ \\
\hline $\begin{array}{c}\text { Hauge \& } \\
\text { Gentile } \\
(2003) \\
\text { USA }\end{array}$ & $\begin{array}{l}\text { Schülerbefra- } \\
\text { gung } \\
\text { 8. \& 9. Jahr- } \\
\text { gangsstufe }\end{array}$ & $\begin{array}{c}\mathrm{N}=607 \\
M=?\end{array}$ & $52 \%$ & $15 \%$ & $\begin{array}{l}7 \text { Items umfassen- } \\
\text { de Abhängigkeits- } \\
\text { skala basierend auf } \\
\text { DSM-IV patholo- } \\
\text { gisches Spielens (5 } \\
\text { von 10) }\end{array}$ \\
\hline $\begin{array}{l}\text { Grüsser et } \\
\text { al. (2005) } \\
\text { Deutsch- } \\
\text { land }\end{array}$ & $\begin{array}{l}\text { Schülerbefra- } \\
\text { gung } \\
\text { 6. Jahrgangs- } \\
\text { stufe }\end{array}$ & $\begin{array}{c}\mathrm{N}=323 \\
M=11.8\end{array}$ & $57 \%$ & $9.3 \%$ & $\begin{array}{l}\text { CSVK, pathologi- } \\
\text { sches Glücksspiel } \\
\text { nach DSM-IV } \\
\text { bzw. ICD-10 }\end{array}$ \\
\hline $\begin{array}{l}\text { Mößle et } \\
\text { al. (2007) } \\
\text { Deutsch- } \\
\text { land }\end{array}$ & $\begin{array}{l}\text { Schülerbefra- } \\
\text { gung } \\
\text { 9. Jahrgangs- } \\
\text { stufe repräsen- } \\
\text { tative Stichpro- } \\
\text { be für } 9 \text { deut- } \\
\text { sche Städte } \\
\text { und Landkreise }\end{array}$ & $\begin{array}{c}\mathrm{N}=14.301 \\
M=15.1\end{array}$ & $50 \%$ & 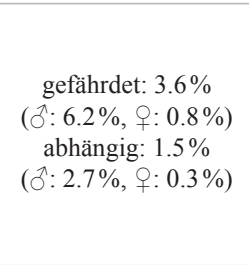 & KFN-CSAS-I \\
\hline $\begin{array}{l}\text { Wölfling } \\
\text { et al. } \\
\text { (2008) } \\
\text { Deutsch- } \\
\text { land }\end{array}$ & $\begin{array}{l}\text { Schülerbefra- } \\
\text { gung } \\
\text { 8. Jahrgangs- } \\
\text { stufe }\end{array}$ & $\begin{array}{c}\mathrm{N}=221 \\
M=14.19\end{array}$ & $48.4 \%$ & $6.3 \%$ & $\begin{array}{l}\text { CSVK-R, patholo- } \\
\text { gisches Spielen } \\
\text { nach DSM-IV } \\
\text { bzw. ICD-10 }\end{array}$ \\
\hline
\end{tabular}




\begin{tabular}{|c|c|c|c|c|c|}
\hline $\begin{array}{l}\text { Autoren } \\
\text { (Jahr) } \\
\text { Land }\end{array}$ & $\begin{array}{l}\text { Methode und } \\
\text { Altersgruppe }\end{array}$ & $\begin{array}{l}\text { Anzahl Befrag- } \\
\text { te }(\mathrm{N}) \text {, Durch- } \\
\text { schnittsalter in } \\
\text { Jahren (M) }\end{array}$ & Anteil $\widehat{\partial}$ & $\begin{array}{l}\text { Anteil abhängige } \\
\text { bzw. gefährdete } \\
\text { Personen }\end{array}$ & $\begin{array}{l}\text { Operationalisie- } \\
\text { rung }\end{array}$ \\
\hline $\begin{array}{l}\text { Rehbein } \\
\text { et al. } \\
(2009 \mathrm{~b} \text {, } \\
2010) \\
\text { Deutsch- } \\
\text { land }\end{array}$ & $\begin{array}{l}\text { Schülerbefra- } \\
\text { gung } \\
\text { 9. Jahrgangs- } \\
\text { stufe } \\
\text { repräsentative } \\
\text { Stichprobe für } \\
\text { Deutschland }\end{array}$ & $\begin{array}{c}\mathrm{N}=15.168 \\
M=15.6\end{array}$ & $51 \%$ & $\begin{array}{c}\text { gefährdet: } 2.8 \% \\
\left(\delta^{+}: 4.7 \%,+ \text {, } 0.5 \%\right) \\
\text { abhängig: } 1.7 \% \\
\left(\delta^{3}: 3.0 \%, \text { + }: 0.3 \%\right)\end{array}$ & KFN-CSAS-II \\
\hline $\begin{array}{c}\text { Gentile } \\
(2009) \\
\text { USA }\end{array}$ & $\begin{array}{c}\text { Onlinebefra- } \\
\text { gung } \\
\text { Altersspanne } \\
8 \text {-18 } \\
\text { repräsentative } \\
\text { Stichprobe für } \\
\text { USA }\end{array}$ & $\begin{array}{c}\mathrm{N}=1.178 \\
M=?\end{array}$ & $50 \%$ & $\begin{array}{c}\text { abhängig: } 8.5 \% \\
\left({ }^{3}: 11.9 \%, \text { + }: 2.9 \%\right)\end{array}$ & $\begin{array}{l}11 \text { Items umfas- } \\
\text { sende Abhängig- } \\
\text { keitsskala basie- } \\
\text { rend auf DSM-IV } \\
\text { pathologisches } \\
\text { Spielen ( } 6 \text { von } 10)\end{array}$ \\
\hline $\begin{array}{l}\text { Wölfling } \\
\text { et al. } \\
\text { (2010b) } \\
\text { Deutsch- } \\
\text { land } \\
\text { Öster- } \\
\text { reich }\end{array}$ & $\begin{array}{l}\text { Schülerbefra- } \\
\text { gung } \\
\text { In NRW und } \\
\text { Wien } \\
\text { 13-18 Jahre }\end{array}$ & $\begin{array}{c}\mathrm{N}=1.710 \\
\text { NRW: } M=14.3 \\
\text { Wien: } M=14.9\end{array}$ & $\begin{array}{l}\text { NRW: } \\
53 \% \\
\text { Wien: } \\
55 \%\end{array}$ & $\begin{array}{l}\text { NRW: } 8.4 \% \\
\text { Wien: } 12.3 \%\end{array}$ & $\begin{array}{l}\text { CSV-S, pathologi- } \\
\text { sches Spielen nach } \\
\text { DSM-IV bzw. } \\
\text { ICD-10 }\end{array}$ \\
\hline $\begin{array}{l}\text { Gentile et } \\
\text { al. (2011; } \\
\text { Choo et } \\
\text { al., 2010) } \\
\text { Singapur }\end{array}$ & $\begin{array}{l}\text { Längsschnitt- } \\
\text { studie Schüler- } \\
\text { befragung im } \\
\text { 2-Jahres-Ab- } \\
\text { stand }\end{array}$ & $\begin{array}{c}\mathrm{N}=2.998 \\
3 .: 737+4 .: 700 \\
M=9.2 \\
7 .: 902+8 .: 658 \\
M=13.0\end{array}$ & $73 \%$ & 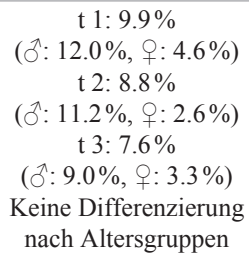 & $\begin{array}{l}10 \text { Items umfas- } \\
\text { sende Abhängig- } \\
\text { keitsskala basie- } \\
\text { rend auf DSM-V } \\
\text { pathologisches } \\
\text { Spielen ( } 5 \text { von } 10)\end{array}$ \\
\hline $\begin{array}{l}\text { Rehbein } \\
\text { et al. } \\
(2011) \\
\text { Deutsch- } \\
\text { land }\end{array}$ & $\begin{array}{l}\text { Schülerbefra- } \\
\text { gung (Berufs- } \\
\text { schüler) } \\
\text { anfallende } \\
\text { Stichprobe }\end{array}$ & $\begin{array}{c}\mathrm{N}=2.667 \\
M=19.8 \\
16-18 \text { Jahre: } \\
991 \AA \\
>\text { 18 Jahre: } \\
1.429 \AA\end{array}$ & $91 \%$ & $\begin{array}{c}\text { Nur } \delta: \\
\text { 16 - 18: gf.: } 1.7 \% \text {, } \\
\text { ah.: } 0.9 \% \\
\text { > 18: gf.: } 0.7 \% \text {, ah.: } \\
0.6 \%\end{array}$ & KFN-CSAS-II \\
\hline $\begin{array}{l}\text { Schmidt, } \\
\text { J.-H. et } \\
\text { al. (2011) } \\
\text { Deutsch- } \\
\text { land }\end{array}$ & $\begin{array}{l}\text { Onlinebefra- } \\
\text { gung } \\
\text { Altersspanne } \\
14-60+(?) \\
\text { repräsentative } \\
\text { Stichprobe für } \\
\text { Deutschland }\end{array}$ & $\begin{array}{c}\mathrm{N}=600 \\
14-19 \text { Jahre: } \\
114\end{array}$ & $57 \%$ & $\begin{array}{c}\text { alle: gf.: } 0.9 \% \text {, ah.: } \\
0.5 \% \\
14 \text { - 19: gf.: } 3.5 \%, \\
\text { ah.: } 0.9 \%\end{array}$ & $\begin{array}{c}\text { modifizierte KFN- } \\
\text { CSAS-II }\end{array}$ \\
\hline
\end{tabular}




\subsubsection{Erklärungsansätze einer Internet- und Computerspielabhängigkeit}

Grüsser und Kollegen (Grüsser, Poppelreuter, Heinz, Albrecht \& Saß, 2007) zeigen mit Referenz auf den aktuellen Forschungsstand, dass ,abgesehen von den sozioökologischen, sozio-strukturellen, soziopolitischen und anthropologischen sowie genetischen Faktoren [...] den psychologischen und neurobiologischen Faktoren bei der Entstehung und Aufrechterhaltung von abhängigem Verhalten eine große Bedeutung zugemessen werden“ muss und gehen von einem „lerntheoretische und neurobiologische Befunde integrierende(n) Ansatz, in dem die Erinnerung an die positive Suchtmittelwirkung als zentraler Motivator für das süchtige Verhalten fungiert" aus (Grüsser et al., 2007, S. 1001). Zur Erklärung der Entstehung und Aufrechterhaltung von substanzungebundenen Verhaltenssüchten wie der Computerspielabhängigkeit spielten dementsprechend Lernprozesse, insbesondere die klassische und operante Konditionierung, eine entscheidende Rolle (Grüsser et al., 2007).

Lernpsychologische Erklärungsansätze. Lernpsychologische Erklärungsansätze stellen Konditionierungsprozesse und Modelllernen in den Vordergrund. Übertragen auf den Gegenstand des Computerspielens bedeutet dies nach Grüsser und Kollegen (Grüsser et al., 2007) beispielsweise, dass im Rahmen einer operanten Konditionierung eine positive Verstärkung durch die angenehme Wirkung des Spielerlebens (Euphorie, Entspannung) und eine negative Verstärkung durch die erfolgreiche Vermeidung bzw. Verdrängung eines vorangegangenen aversiven, zur Aufnahme des Spielverhaltens motivierenden Erlebniszustandes (z. B. Anspannungszustände oder Entzugserscheinungen) erfolgt. Infolgedessen werden vormals neutrale Reize (z. B. der Anblick einer Spielkonsole) und die zur Aufnahme des belohnenden Verhaltens motivierende Erlebniszustände wie erhöhter Stress oder realweltliche Misserfolge immer stärker mit dem Spielverhalten verknüpft und können damit als ,suchtassoziierte Reize“ zu einer regelmäßigen Aufnahme des abhängigen Verhaltens motivieren (klassische Konditionierung) (Grüsser et al., 2007). Diese Prozesse unterliegen einem selbstverstärkenden Kreislauf, indem intrapsychische (z. B. negatives Selbstbild), psychovegetative (Entzugserscheinungen) und psychosoziale Konsequenzen der Abhängigkeit (z. B. Leistungseinbrüche in der Schule, Probleme in Sozialbeziehungen) jeweils mit aversiven Gefühlen einhergehen, die das Verlangen nach dem Verhalten verstärken. Eine erneute exzessive Ausübung des Verhaltens erhöht in Folge wiederum diese auslösenden Bedingungen. Bei der Entstehung dieses Kreislaufes könnte auch der innerfamiliäre Umgang mit Medien eine entscheidende Rolle spielen. Wenn Medienwelten durch die Eltern schon frühzeitig als Ersatzwelten für ausgebliebene realweltliche Erlebnisse und Beziehungserfahrungen nahegelegt werden und dieser Umgang durch die Eltern vorgelebt wird, könnten im Sin- 
ne des Modelllernens die heranwachsenden Kinder diesen Umgang fortführen und sich damit auch einem höheren Risiko dafür aussetzen, eine Abhängigkeit von virtuellen Welten zu entwickeln (vgl. Grüsser, S. M. \& Thalemann, C. N., 2006).

Einen erweiterten lernpsychologischen Erklärungsrahmen, der insbesondere geeignet erscheint, interindividuelle Unterschiede in der Abhängigkeitsprädisposition zu erklären, bilden kognitive Erklärungsansätze. Diese beziehen gefestigte Überzeugungen wie zum Beispiel verringerte Selbstwirksamkeitserwartungen (die Überzeugung, Problemen im Alltag nicht angemessen begegnen zu können), Wirkungserwartungen hinsichtlich des belohnenden Verhaltens (antizipierte negative und positive Effekte) und dysfunktionale Grundüberzeugungen (z. B. „Nur schwache Menschen werden abhängig von Computerspielen“) in die Überlegungen zur Entstehung von Abhängigkeit ein. Dysfunktionale Grundannahmen könnten als rigide und überdauernde kognitive Strukturen eine besondere Belohnungserwartung durch oder Legitimierung für das eigene Spielverhalten beinhalten, was seinen Ausdruck in einer erhöhten Anfälligkeit für die Entwicklung von Abhängigkeitserkrankungen und einer erhöhten Rückfallneigung finden könnte (vgl. Tiffany, 1990; 1995, zitiert nach Grüsser \& Thalemann, 2006).

Neurobiologische Erklärungsansätze. Neurobiologische Erklärungsansätze betonen in Analogie zum pathologischen Glücksspiel sowie zur Substanzabhängigkeit die Rolle des in verschiedenen menschlichen Hirnregionen lokalisierten, körpereigenen Belohnungssystems und die sich hieraus ergebenen verhaltensstimulierenden Wirkungen. Dabei dürften prädispositionell genetische Faktoren eine wichtige Rolle spielen, die bei computerspielabhängigen Personen vergleichbar zu anderen Abhängigkeitserkrankungen Auffälligkeiten im dopaminergen Belohnungssystem und der serotonergen Übertragung erwarten lassen. In einem Modell von Bergmann und Hüther (2006) spielen dabei insbesondere das im Zwischenhirn lokalisierte „Belohnungssystem“ sowie die neurochemische Vermittlung durch Dopamin und endogene Opiate eine tragende Rolle. Als neuroplastischer Botenstoff stimuliert Dopamin nicht nur die Freisetzung endogener Opiate, sondern führt auch zu einer Bahnung und Stärkung jener neuronaler Verbindungen, welche, ,gleichzeitig aktiviert werden, wenn man die betreffende, so extrem befriedigende Tätigkeit - also z. B. das Computerspielen oder das Joggen - ausübt" (Bergmann \& Hüther, 2006, S. 125). Als biochemisches Endprodukt der Belohnung erzeugen die endogenen Opiate das eigentliche Lustgefühl einer dadurch auch als extrem befriedigend erlebten Handlung. Nach Bergmann und Hüther (2006) ist dieses ,subjektive empfundene Gefühl, dieses zufriedenstellende Gefühl der Befriedigung“ (Bergmann \& Hüther, 2006, S. 125) wie für alle suchtartigen Verhaltensweisen auch notwendige Voraussetzung für die Ausbildung einer Computerspielabhängigkeit. Im Speziellen „wird die Wiederholung 
bestimmter virtueller Erfahrungen, die mit ausgeprägt positiven Empfindungen einhergeht (Opiatausschüttung), aus der Gewöhnung und Erwartung heraus (Dopaminausschüttung) zu neuronal gefestigten Bahnungen bestimmter Empfindungs-, Denk- und Verhaltensmuster führen“ (te Wildt, 2009, S. 268). Hier wird noch einmal die Wichtigkeit einer integrativen Betrachtung lerntheoretischer und neurobiologischer Ansätze zur Erklärung einer Computerspielabhängigkeit deutlich (vgl. Grüsser et al., 2007; te Wildt, 2009). Dies lässt sich in der Erklärung der Aufrechterhaltung einer Abhängigkeit zusätzlich verdeutlichen. So konnte z. B. gezeigt werden, dass Konditionierungsprozesse mit einer Adaption neurobiologischer Strukturen (neurochemische Sensitivierung) einhergehen, die sich mit bildgebenden Verfahren nachweisen lassen und als physiologisches Substrat eines impliziten „Suchtgedächtnisses“ verstanden werden können. Hier wird u. a. eine mögliche Erklärung dafür gesehen, warum nach jahrelanger Abstinenz bereits die einmalige Konfrontation mit dem Suchtmittel wieder zur Aufnahme des abhängigen Verhaltens führen kann (Grüsser, S. M. \& Thalemann, C. N., 2006).

Bei Betrachtung lernpsychologischer oder neurobiologischer Erklärungsansätze einer Computerspielabhängigkeit darf die das exzessive Verhalten stimulierende "Substanz“ auf die das menschliche Belohnungssystem trifft nicht außer Acht gelassen werden. Warum werden bestimmte Computerspiele als besonders belohnend erlebt? Insbesondere in komplexen Spielkonzeptionen wie Onlinerollenspielen, die vernetzt im Internet gespielt werden können, sind zunächst die Belohnungen für den Spieler besonders ausdifferenziert und erfolgen auf unterschiedlichen Ebenen. So finden sich z. T. gleichzeitig Belohnungen in Form von Punkten, Ranglistenplatzierungen, einem Voranschreiten der Spielgeschichte, frei gespielten Zwischensequenzen und Bonusgegenständen, neuen Fähigkeiten oder Gestaltungsmöglichkeiten der Spielfigur, einer Entdeckung von exklusiven Geheimnissen und einer zunehmenden Anerkennung durch Mitspieler; all dies kann zum Weiterspielen motivieren und ein intensives Belohnungserlebnis beim Nutzer hervorrufen. Wie am Beispiel von World of Warcraft (WoW) bereits dargestellt wurde (vgl. Kapitel 2.2.3, Fußnote 78), können sich die unterschiedlichen Belohnungsebenen in Onlinespielen dabei untrennbar vermischen: Virtuelle Belohnungen werden unmittelbar zu sozial relevanten Prestigeobjekten in der Spielergemeinschaft und bestimmen damit den sozialen Status und die Handlungsmöglichkeiten des Spielers in seiner virtuellen Welt maßgeblich. Virtuellen Belohnungen kommt somit nicht nur eine abstrakte auf das Spiel bezogene Bedeutung zu, sie können vielmehr auch eine über das Spiel hinausgehende identitätsstiftende Relevanz für den Spieler einnehmen.

Neben der Vielschichtigkeit und Ausdifferenzierung der Belohnungsvergabe, erfolgt diese in Onlinerollenspielen wie $W o W$ zudem in besonderer Weise: Für den Spieler ist der weitere Verlauf eines Spiels und die Frage, ob ein folgender 
Spielabschnitt erfolgreich gemeistert wird - ob und wann eine bestimmte Belohnung erfolgt - in der Regel nicht vorhersehbar. Damit ähnelt die Belohnungsvergabe konzeptionell einer intermittierenden Verstärkung im Sinne eines variablen Quotenplans, einer lernpsychologischen Verhaltensbedingung, die ein immer wiederkehrendes und intensives Verhalten qua Konzeption begünstigt, da im Gegensatz zur kontinuierlichen Verstärkung nicht jede, sondern nur manche Verhaltensweisen belohnt werden. ${ }^{168}$ Nach lernpsychologischen Erkenntnissen erzeugen Verstärkerpläne dieser Art die höchsten Reaktionsraten und weisen die größte Löschungsresistenz auf. Somit wird ein exzessiv belohnendes Verhalten in besonderer Weise begünstigt, weshalb variable Quotenpläne auch als das suchtendogene Element von Glücksspielen gelten (vgl. Griffiths, 2008). Glücksspiele und Computerspiele haben somit diesbezüglich eine große konzeptionelle, psychologische und verhaltensstimulierende Ähnlichkeit und können demzufolge für manche Personen ein ähnliches psychotropes Abhängigkeitsrisiko bergen (vgl. Griffiths, 2008).

Aufgrund der dargestellten Ergebnisse lässt der bisherige Forschungsstand zur Computerspielabhängigkeit sowie zu ihren Referenzerkrankungen, allen voran dem Pathologischen Glücksspiel, insgesamt auf eine multifaktorielle Genese schließen. Abbildung 70 zeigt den Versuch, diese multifaktorielle Genese in einem Erklärungsmodell der Computerspielabhängigkeit unter Berücksichtigung der Risikokorrelate und Komorbiditäten zusammenzufassen. Die hier skizzierten neurobiologischen und psychosozialen Faktoren, die eine Computerspielabhängigkeit bedingen und aufrechterhalten können, zeigen auch unter der Berücksichtigung der regelhaft auftretenden komorbiden Störungen noch einmal an, wie tiefgreifend diese Form von Abhängigkeit sein kann.

168 In MMORPGs wie $W o W$ werden Spieler mit komplexen, mehrstündigen Aufgaben (Quests) konfrontiert, die nur gemeinsam mit einer großen Zahl von Mitspielern (der Gilde) $\mathrm{zu}$ bewältigen sind. Am Ende eines solchen Quests lässt in der Regel ein besiegter Endgegner zufällig einen von mehreren möglichen und besonders wertvollen und prestigeträchtigen Gegenständen fallen. Die Spieler können sich zwar vorab in Foren oder Ratgeberbüchern darüber informieren, welche Gegenstände das sein können und mit welcher Wahrscheinlichkeit sie fallen gelassen werden, trotzdem lässt sich das genaue Geschehen nicht voraussagen, zumal seltene und wichtige Gegenstände derart begehrt sind, dass sie in der Regel am Ende eines Quests innerhalb der Gilde meistbietend versteigert werden. So kommt es regelmäßig dazu, dass Spieler bestimmte Quests immer wieder in Angriff nehmen müssen, um in den Besitz bestimmter seltener für sie wichtiger Gegenstände zu kommen. Damit weisen Spielkonzepte wie $W o W$ ein typisches Kennzeichen variabler Quotenpläne auf: Qua Konzeption begünstigen sie ein immer wiederkehrendes und intensives Verhalten. 


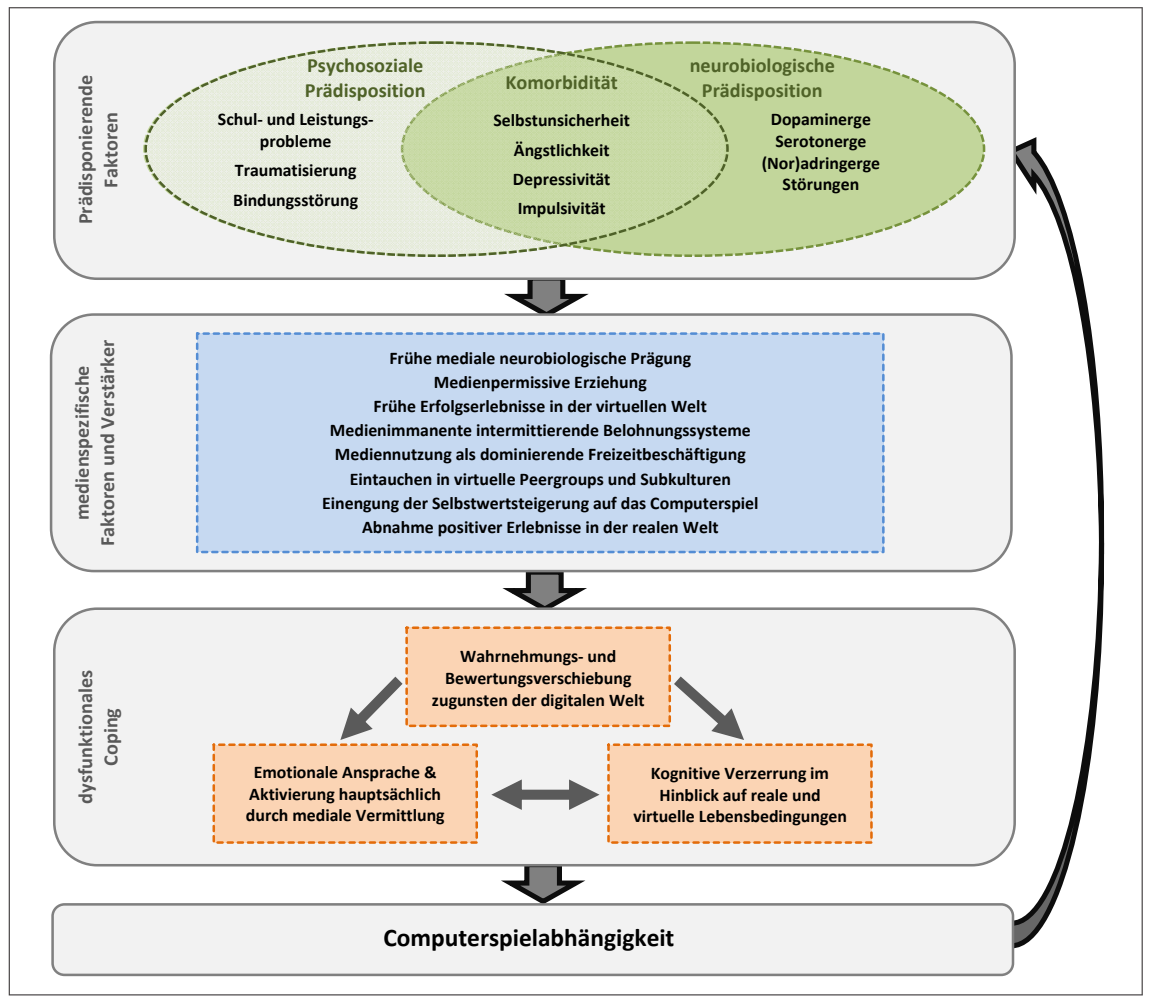

Abbildung 70. Multifaktorielles Erklärungsmodell einer

Computerspielabhängigkeit. ${ }^{169}$

5.2 Ergebnisse des Berliner Längsschnitt Medien - Computerspielabhängigkeit

Im Mai 2008 (5. Klasse) wurde das Thema Computerspielabhängigkeit zum ersten Mal in die Befragungen des Berliner Längsschnitts Medien mit aufgenommen. Zur empirischen Überprüfung des Risikos einer Computerspielabhängigkeit wurde zunächst die Skala Computerspielabhängigkeit der Schülerbefragung 2005 - KFN-CSAS-I (Grundlage bei der Entwicklung war die ISS-20; Hahn \& Jerusalem, 2001c; Rehbein \& Borchers, 2009; Mößle et al., 2007), später deren Weiterentwicklung, die KFN-CSAS-II (vgl. Rehbein et al., 2009a, 2009b, 2010) eingesetzt (vgl. Kapitel 1.5.1, Tabelle 19). ${ }^{170}$ In der KFN-Schülerbefragung 2005

169 Das Modell basiert auf einem Modell der Medienabhängigkeit von Te Wildt (2010).

170 Die beiden Skalen korrelieren mit $r=.95$ miteinander (Rehbein et al., 2009a, 2009b, 2010). 
konnten mit Hilfe der KFN-CSAS-I etwa fünf Prozent und in der KFN-Schülerbefragung 2007/2008 mit Hilfe der KFN-CSAS-II etwa viereinhalb Prozent der im Durchschnitt 15-jährigen Jugendlichen als computerspielabhängig bzw. abhängigkeitsgefährdet eingestuft werden (Baier \& Rehbein, 2009; Mößle et al., 2007).

Der Berliner Längsschnitt Medien ermöglicht nun die Beantwortung einiger wichtiger Fragen: Handelt es sich bei Computerspielabhängigkeit um ein reines Jugendphänomen oder können bei im Durchschnitt 11.5-jährigen Schülerinnen und Schülern der fünften Klasse sowie im Durchschnitt 12.5-jährigen Schülerinnen und Schülern der sechsten Klasse, auch bereits Kinder identifiziert werden, die in diesem Alter die klinischen Merkmale einer Computerspielabhängigkeit zeigen? (vgl. Kapitel 5.2.2) Können ausgehend vom internationalen Forschungsstand bei Betrachtung der Genese einer Computerspielabhängigkeit neben medienspezifischen Risikokorrelaten auch besondere Faktoren des sozialen bzw. schulischen Umfeldes oder der Persönlichkeit ermittelt werden, die Kinder für die Entwicklung einer Computerspielabhängigkeit prädisponieren? Welche Risikokorrelate eignen sich dabei insbesondere zur längsschnittlichen Vorhersage? (vgl. Kapitel 5.2.3, 5.2.4) Können bereits im Kindesalter psychische Auffälligkeiten wie eine depressive Verstimmung oder Hyperaktivität, die später als Komorbiditäten einer Computerspielabhängigkeit berichtet werden, vermehrt bei computerspielabhängigen Kindern beobachtet werden? (vgl. Kapitel 5.2.3, 5.2.4) Gehen diese möglicherweise einer Computerspielabhängigkeit voraus oder sind sie deren Folge? (vgl. Kapitel 5.2.5)

\subsubsection{Zeitlich exzessives Computerspielverhalten}

Einen ersten Einblick in den Anteil derjenigen Kinder mit problematischem Spielverhalten ermöglicht die Betrachtung der Computerspielnutzungszeiten. Die Auswertungen wurden dabei basierend auf der sog. Schätzfrage (siehe Kapitel 1.5.1, vgl. Abbildung 7) durchgeführt. 


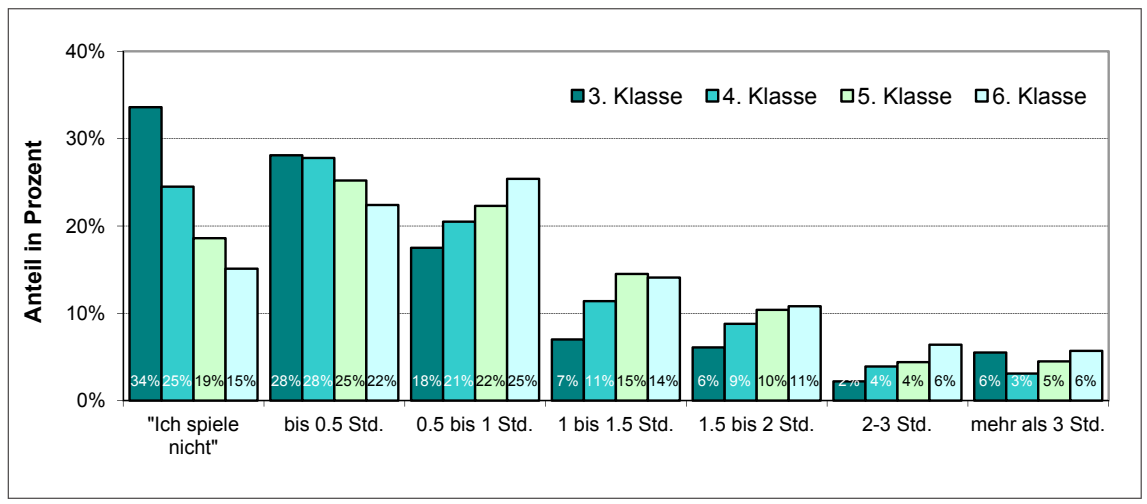

Abbildung 71. Anteil an zeitlichen Nutzergruppen von Computerspielen an einem Schultag getrennt nach Jahrgangsstufe (3., 4., 5. und 6. Klasse).

Betrachtet man die Angaben aller Kinder für einen Schultag (siehe Abbildung 71), fällt zunächst auf, dass der Anteil derjenigen Kinder, die angeben an einem Schultag keine Computerspiele zu spielen, mit dem Alter deutlich abnimmt. Waren es am Ende der dritten Klasse noch 34 Prozent der computerspielenden Kinder, sind es am Ende der sechsten Klasse nur noch 15 Prozent, die an einem Schultag gar keine Computerspiele spielen. In Abbildung 71 wurden nur diejenigen Kinder mit aufgenommen, die die Frage „Spielst du überhaupt Computeroder Videospiele, auch wenn es ganz selten ist" bejahten. ${ }^{171}$ Schaut man auf das andere Ende der Verteilung (mehr als 3 Std.), so schwankt der Anteil der zeitlich exzessiv computerspielenden Kinder, zwischen drei und sechs Prozent. Mit mehr als drei Stunden täglicher Computerspielzeit an einem Schultag wäre die Grenze für exzessives Computerspielen für diese Altersgruppe zugegebener Maßen sehr konservativ gesetzt. Nimmt man noch die Kinder, die angeben zwei bis drei Stunden an einem Schultag Computerspiele zu spielen hinzu, liegt der Anteil exzessiv spielender Kinder zwischen sieben (in der vierten Klasse) und zwölf Prozent (in der sechsten Klasse) und entspricht eher einer empirisch basierten Definition von exzessivem Computerspielen (vgl. Mößle et al., 2007), nach der die oberen zehn Prozent der Verteilung als Exzessivspieler eingestuft werden. Gemäß dieser Definition könnten in der dritten und vierten Klasse Kinder, die zwischen einer und zwei Stunden, in der fünften und sechsten Klasse, Kinder die eineinhalb bis zwei Stunden täglich Computerspiele spielen, als Vielspieler bezeichnet werden. In der dritten und vierten Klasse würden sich Kinder mit tägli-

171 In der dritten Klasse waren dies 84 Prozent, in der vierten und fünften Klasse jeweils 92 Prozent und in der sechsten Klasse 90 Prozent der Schülerinnen und Schüler. 
chen Computerspielzeiten von bis zu einer Stunde, in der fünften und sechsten Klasse von bis zu eineinhalb Stunden im Normalbereich bewegen. Insgesamt verschiebt sich die Verteilung der Computerspielzeiten zwar erst über die Jahrgangsstufen hin zu höheren Nutzungszeiten, es kann aber bereits in der dritten Klasse ein bestimmter Anteil von Kindern identifiziert werden, der exzessiv Computerspiele spielt. Von entscheidender Bedeutung ist nun die Frage, ob diese Kinder, die bereits sehr früh sehr viel Zeit mit Computerspielen verbringen, später auch in der Diagnostik einer Computerspielabhängigkeit auffällig werden.

\subsubsection{Problematisches Computerspielverhalten - Computerspielabhängigkeit}

Insgesamt ergeben sich bei Verwendung der KFN-CSAS-I (vgl. Mößle et al., 2007; Rehbein \& Borchers, 2009) auf Basis der Daten aus der fünften Klasse einbezogen wurden alle Kinder, die an der Befragungswelle teilgenommen haben, also auch Kinder die keine Computerspiele spielen ( $8 \%$ der Kinder der fünften Klasse) - die folgenden Prävalenzschätzungen für Computerspielabhängigkeit: 2 Prozent der Schülerinnen und Schüler sind als abhängigkeitsgefährdet ( $0.3 \%$ der Mädchen, $3.7 \%$ der Jungen) und 0.7 Prozent der Schülerinnen und Schüler als abhängig einzustufen ( $0 \%$ der Mädchen, $1.5 \%$ der Jungen). In der sechsten Klasse - einbezogen wurden wiederum alle Kinder, auch Kinder die keine Computerspiele spielen (10\% der Kinder der sechsten Klasse) - ergeben sich bei Verwendung der KFN-CSAS-II (vgl. Rehbein et al., 2009a, 2009b, 2010) folgende Prävalenzschätzungen für Computerspielabhängigkeit: 2.1 Prozent der Schülerinnen und Schüler sind als abhängigkeitsgefährdet $(0.8 \%$ der Mädchen, $3.4 \%$ der Jungen) und 1.9 Prozent der Schülerinnen und Schüler als abhängig einzustufen ( $0.5 \%$ der Mädchen, 3.2\% der Jungen). Somit ist von der fünften zur sechsten Klasse eine Zunahme an Schülerinnen und Schülern, die als computerspielabhängig diagnostiziert werden zu beobachten, die vor allem bei den Jungen ihre Ursache findet.

Bei einer Stichprobengröße von 807 bzw. 806 Schülern und Schülerinnen, die jeweils die Schülerfragebögen im Mai 2008 bzw. Mai 2009 ausfüllten, führt dies naturgemäß zu recht kleinen Fallzahlen, die jedoch aufgrund der Stichprobenziehung nach Maßgabe strenger Randomisierung immer noch repräsentativen Anspruch haben. Fasst man die beiden Kategorien abhängig und gefährdet zu einer zusammen, werden im Mai 2008 (5. Klasse) 21 Schüler und 1 Schülerin als im klinischen Sinn pathologische Computerspieler auffällig, im Mai 2009 (6. Klasse) sind dies 27 Schüler und 5 Schülerinnen. Wie zu erwarten, liegen diese Prävalenzschätzungen somit unter denen der 15-Jährigen aus der KFN-Schülerbefragung 2005 (etwa 5 Prozent computerspielabhängige bzw. abhängigkeitsge- 
fährdete Computerspieler; vgl. Mößle et al., 2007) sowie der KFN-Schülerbefragung 2007/2008 (etwa 4.5 Prozent computerspielabhängige bzw. abhängigkeitsgefährdete Computerspieler, vgl. Rehbein et al., 2009a, 2009b, 2010), erreichen aber dennoch bereits in der fünften Klasse ein substanzielles Niveau. So liegt zum Vergleich die Prävalenz der Aufmerksamkeitsdefizit-/Hyperaktivitätsstörung (ADHS) laut dem deutschen Kinder- und Jugendgesundheitssurvey im Grundschulalter bei 5.3 Prozent (7 bis 10 Jahre) bzw. bei 7.1 Prozent bei 11bis 13-jährigen Kindern (Schlack, Hölling \& Huss, 2007).

Hinsichtlich der Frage der Stabilität des Störungsbildes Computerspielabhängigkeit konnte zudem im Berliner Längsschnitt Medien der Nachweis einer gewissen Dauerhaftigkeit erbracht werden. Bei einer insgesamt hohen Korrelation $(r=.52)$ der Skalenwerte Computerspielabhängigkeit der beiden Messzeitpunkte waren 56 Prozent der in der fünften Klasse als problematisch (gefährdet oder abhängig) klassifizierten Schülerinnen und Schüler dies auch noch nach einem Jahr in der sechsten Klasse: von 18 Schülerinnen und Schülern, die in der fünften Klasse als problematische Computerspieler und -spielerinnen identifiziert wurden und zu denen in der sechsten Klasse Angaben vorlagen, wurden 10 weiterhin als problematische Computerspieler und -spielerinnen klassifiziert. Demgegenüber wurden aber auch während der einjährigen Befragungsdauer von der fünften zur sechsten Klasse weitere drei Prozent der Schülerinnen und Schüler zusätzlich als computerspielabhängig eingestuft: von 597 Schülerinnen und Schülern, die in der fünften Klasse als unproblematische Computerspieler und -spielerinnen identifiziert wurden und zu denen in der sechsten Klasse Angaben vorlagen, wurden 18 als problematische bzw. suchtartige Computerspieler und -spielerinnen klassifiziert.

Betrachtet man die unterschiedlichen Nutzergruppen hinsichtlich einer Computerspielabhängigkeit, und zwar auf Basis der Computerspielnutzungszeiten der sechsten Klasse, ergibt sich das folgende Bild: Bei den Normalspielern (bis zu 1.5 Stunden tägliche Nutzungszeit an einem Schultag) sind nur 0.9 Prozent als gefährdet oder abhängig einzustufen. Bei den Vielspielern (1.5 bis 2 Stunden tägliche Nutzungszeit) wird das Phänomen Computerspielabhängigkeit bereits relevant: Sieben Prozent der Kinder werden in dieser Nutzergruppe als gefährdet oder abhängig eingestuft. In der Gruppe der Exzessivspieler (mehr als 2 Stunden tägliche Nutzungszeit) sind es schließlich 23 Prozent der Kinder, die als im klinischen Sinne pathologische Computerspieler bezeichnet werden können. Vergleicht man hierzu die Nutzungszeiten der Kinder in der dritten Klasse, so zeigt sich, dass diejenigen Kinder die bereits zu diesem frühen Zeitpunkt hohe Computerspielnutzungszeiten (bereits im Bereich der Vielspieler) aufweisen, auch besonders häufig in der sechsten Klasse als computerspielabhängig oder abhängigkeitsgefährdet identifiziert werden. Liegt der Anteil der auffälligen Schülerinnen 
und Schüler bei den Normalspielern (bis zu 1 Stunde tägliche Nutzungszeit an einem Schultag) der dritten Klasse bei 2.5 Prozent, steigt er bereits bei den Vielspielern (1 bis 2 Stunden tägliche Nutzungszeit) der dritten Klasse auf 12.3 Prozent. In der Gruppe der Exzessivspieler (mehr als 2 Stunden tägliche Nutzungszeit) der dritten Klasse ist es schließlich beinahe jedes zehnte Kind (9\%), das in der sechsten Klasse ein auffälliges Computerspielverhalten zeigt.

Dieser Zusammenhang wird auch noch einmal in Abbildung 72 verdeutlicht. Aufgenommen wurden nur Kinder, die in der fünften oder sechsten Klasse als gefährdete oder abhängige Computerspieler klassifiziert wurden. ${ }^{172}$ Zunächst wird recht augenscheinlich deutlich, dass sich die Verteilung der auffälligen Computerspieler genau entgegengesetzt zur Verteilung aller computerspielenden Kinder verhält (vgl. Abbildung 71), d. h., auffällige Computerspieler bewegen sich am oberen Ende der Verteilung der abgefragten Nutzungszeiten und dies bereits am Ende der dritten Klasse. So berichtet fast ein Drittel der Kinder in der Gruppe der abhängigen und gefährdeten Computerspieler bereits in der dritten Klasse über ein zeitlich exzessives Computerspielverhalten. Die Verteilung zeigt aber auch, dass nicht alle Kinder, die ihr Computerspielverhalten als abhängig empfinden, exzessive Nutzungszeiten an Schultagen haben. ${ }^{173}$

172 Für die Darstellung nach zeitlichen Nutzergruppen in der sechsten Klasse wurden lediglich Schülerinnen und Schüler berücksichtigt, die in der sechsten Klasse als abhängigkeitsgefährdet oder als abhängig diagnostiziert wurden.

173 Für diejenigen Kinder, die angaben an einem Schultag nicht zu spielen, müsste hier hinterfragt werden, wie dies mit einer Einstufung als abhängig oder gefährdet in der fünften oder sechsten Klasse zusammenpasst. Eine mögliche Erklärung zumindest für die dritte und vierte Klasse besteht in einer Veränderung der Computerspielzeiten in dieser Gruppe. So ist der Prozentsatz in der fünften und sechsten Klasse auch deutlich geringer. Möglicherweise werden hier auch durch die Zusammenfassung der Angaben der fünften und sechsten Klasse zu einer Kategorie bestehende Unterschiede überdeckt. 


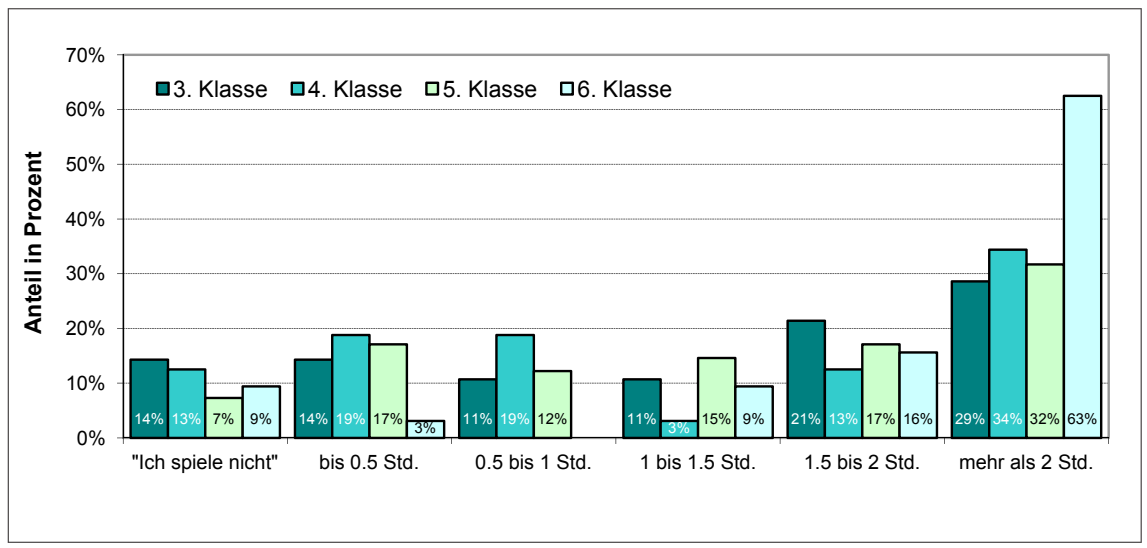

Abbildung 72. Anteil an zeitlichen Nutzergruppen von Computerspielen an einem Schultag getrennt nach Jahrgangsstufe (3., 4., 5. und 6. Klasse). Nur gefährdete und abhängige Computerspieler.

\subsubsection{Risikokorrelate und Komorbiditäten einer Computerspielabhängigkeit}

Um die Gruppe der auffälligen (gefährdeten oder abhängigen) Computerspieler in Abgrenzung zu den unauffälligen Computerspielern differenzierter beschreiben zu können, sollen zunächst unterschiedliche Variablen der Mediennutzung betrachtet werden (vgl. Tabelle 70 und Abbildung 73) bevor auf Faktoren des schulischen Umfeldes (vgl. Tabelle 71), der Persönlichkeit (vgl. Tabelle 72) und des sozialen Umfeldes (vgl. Tabelle 73) eingegangen wird, die Kinder möglicherweise für die Entwicklung einer Computerspielabhängigkeit prädisponieren. ${ }^{174}$

Mediennutzungsspezifische Risikokorrelate

Medienausstattung. Hinsichtlich der Ausstattung mit einem eigenen PC fällt zunächst auf, dass sich die auffälligen Computerspieler nur hinsichtlich des PCBesitzes in der dritten Klasse bedeutsam von den unauffälligen Computerspielern unterscheiden; sie haben zu diesem frühen Zeitpunkt deutlich häufiger einen eigenen PC im Kinderzimmer. Ähnlich stellt sich das Bild bezüglich der eigenen Spielkonsole dar: Während nur 42 Prozent der unauffälligen Computerspieler in

174 Skalen und Testinstrumente sind Kapitel $1.5 \mathrm{zu}$ entnehmen. 
der dritten Klasse eine eigene Spielkonsole im Kinderzimmer hatte, waren dies bereits 22 von 28 (79\%) der abhängigen oder gefährdeten Computerspieler. Ein Unterschied, der bis zur sechsten Klasse stabil bleibt. Die größeren Ausstattungsdifferenzen mit Spielkonsolen sind erwartungsgemäß, da zum einen Kinder insgesamt erst später mit ihrem eigenen PC ausgestattet werden als mit der eigenen Konsole und zum anderen Computerspiele in dieser Altersgruppe noch primär an tragbaren und stationären Konsolen gespielt werden. Bis zum Ende der sechsten Klasse ist die Ausstattung mit beiden Geräten jedoch annähernd vergleichbar.

Computerspielzeiten. Die als auffällig diagnostizierten Computerspieler weisen bereits in der dritten Klasse mit 83 Minuten mehr als doppelt so hohe tägliche Computerspielzeiten auf als die Vergleichsgruppe der unauffälligen Computerspieler. Dieser Unterschied verfestigt sich in den Folgejahren und wird in der vierten, fünften und sechsten Klasse auch statistisch bedeutsam. Hinsichtlich der Computerspielzeiten der Eltern zeigen sich ebenfalls zum Teil deutliche Unterschiede in den Nutzungszeiten der Väter und Mütter computerspielabhängiger oder gefährdeter Kinder. Dieser Unterschied ist aber mit Vorsicht zu interpretieren, da in der Gruppe der auffälligen Kinder nur Angaben zu maximal 13 Eltern vorlagen. Auf die Bedeutung der elterlichen Computerspielzeiten soll zum Ende des Kapitels noch etwas differenzierter eingegangen werden.

Spielmotive. Computerspieler mit einem auffälligen Computerspielverhalten neigen auch dazu, Computerspiele insbesondere dann zu nutzen, wenn es in ihrem Leben gerade ,nicht so gut läuft“. Dieser Unterschied fällt in der sechsten Klasse noch größer als in der fünften Klasse aus.

Computerspielpräferenzen. Bedeutsame Unterschiede zwischen den Gruppen ergeben sich auch hinsichtlich der Präferenz der Befragten für bestimmte Computerspielgenres ${ }^{175}$ (abgefragt in der 5. und 6. Klasse). Die größten Unterschiede zwischen unauffälligen Computerspielern und auffälligen Spielern zeigen sich bei den Präferenzen für Prügelspiele, First- und Third-Person-Shooter sowie Onlinerollenspiele. Dabei erscheint insbesondere die Vorliebe der abhängigen bzw. abhängigkeitsgefährdeten Spieler für Onlinerollenspiele problematisch, schließlich sind diese mit einer Altersfreigabe von in der Regel ,ab 12 Jahren“ für einige der untersuchten Schüler der fünften Klasse und spätestens in der sechsten Klasse bereits „legal“ zu erwerben. Das beliebteste Onlinerollenspiel unter Schülern mit problematischem Computerspielverhalten ist das Spiel WoW, welches ,ab 12 Jahren“ freigeben ist, während die genannten Shooter je nach Ti-

175 Die Variable Genrepräferenz wurde wie folgt gebildet: Schüler, die auf die Frage ,wie häufig spielst du die folgenden Arten von Computer- oder Videospielen“ „nie“ oder ,,selten“ ankreuzten haben keine Präferenz für dieses Genre. Schüler, die ,sehr oft“ ankreuzten haben eine Präferenz. Haben die Schüler „manchmal“ oder ,sehr oft“ angekreuzt besteht eine Genrepräferenz, wenn kein weiteres Genre öfter gespielt wird (vgl. Kapitel 1.5.1). 
tel Altersfreigaben „ab 16 Jahren“ oder „ab 18 Jahren“ oder eine Indizierung erhalten haben. Obwohl beide Spieltypen im Zusammenhang mit zeitlich problematischem und pathologischem Spielverhalten von Grundschülern zu sein scheinen, entscheidet allein der höhere Anteil expliziter Gewalt über die rigidere Alterseinstufung.

Betrachtet man die Spielvorlieben der Befragten aufgrund der ebenfalls erhobenen Lieblingstitel, bestätigt sich die Vorliebe für First- und Third-PersonShooter sowie für Onlinerollenspiele. So sind die beiden beliebtesten Spiele unter Schülerinnen und Schülern der sechsten Klasse mit auffälligem Computerspielverhalten ${ }^{176}$ zwar, wie bereits für die männliche Stichprobe berichtet, die FIFA-Reihe mit acht Nennungen sowie GTA mit sieben Nennungen. In Abweichung zur Top Ten aller Jungen finden sich auf den weiteren Plätzen jedoch der First-Person-Shooter Counterstrike (6 Nennungen) sowie die beiden Onlinerollenspiele $W o W$ (5 Nennungen), welches es nicht in die Top Ten aller männlichen Sechstklässler schaffte, und Metin 2 (3 Nennungen).

Nutzung und Bezug altersinadäquater Computerspiele (vgl. Kapitel 2.2.3). An den Lieblingsspieletiteln zeigt sich auch die besondere Vorliebe der computerspielabhängigen und gefährdeten Computerspieler für gewaltbetonte Spiele, welche bereits in den Genrepräferenzen deutlich geworden ist. Angesichts der geringen Fallzahl von 44 Personen und ca. 60 unterschiedlichen Spieletiteln in der Gruppe der auffälligen Spieler, ist hier ein Vergleich der Einzeltitel der verschiedenen Gruppen in einer Rangreihe wenig gewinnbringend. Es fällt jedoch auf, dass auffällige Computerspieler insgesamt weitaus häufiger im Vergleich zur Gegengruppe ein Computerspiel als eines ihrer momentanen drei Lieblingsspiele nennen, welches aufgrund der dargestellten Gewalt von der USK ab 16 Jahren freigegeben wurde, keine Jugendfreigabe oder keine Kennzeichnung erhalten hat (vgl. Abbildung 73). 


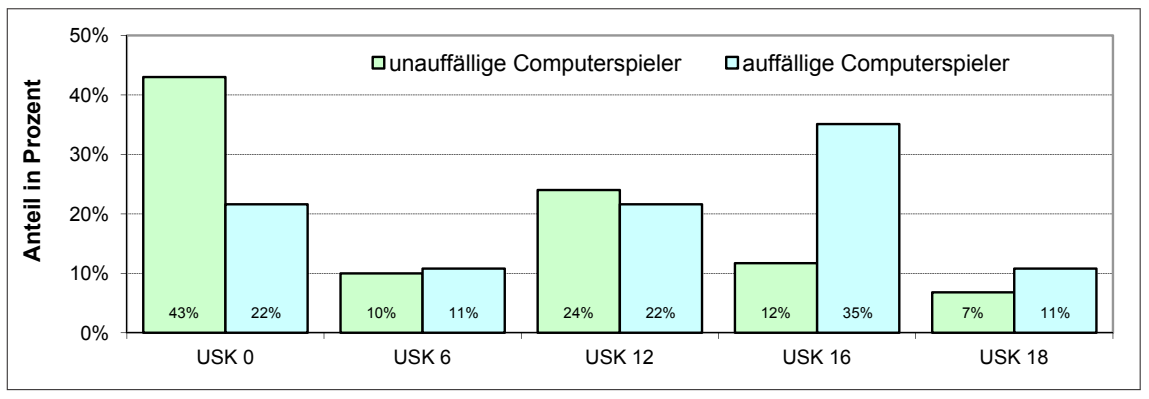

Abbildung 73. Anteil an Computerspielnutzergruppen nach auffälligem Computerspielverhalten. ${ }^{177}$

Von den Spielkonstellationen (alleine, mit Freunden, mit Vater, mit Mutter, mit anderen Erwachsenen) wird zwar fast jede Konstellation zu jedem Messzeitpunkt von den gefährdeten und abhängigen Spielern häufiger angegeben, das Spielen mit Freunden und insbesondere das Spielen alleine, dessen Gruppenunterschied bereits in der dritten Klasse ein statistisches bedeutsames Niveau erreicht, scheinen jedoch besonders auffällig zu sein.

Medienerziehung. Die häufige Nutzung von altersinadäquaten Computerspielen ist jedoch nicht nur ein Indikator für problematisches Spielverhalten, sondern könnte auch ein Kennzeichen für weitere persönliche und/oder soziale Probleme sein. Die Nutzung derartiger Inhalte lässt auf mangelnde Zuwendung und Beaufsichtigung durch die Eltern schließen, die ebenso für die Entstehung von Computerspielabhängigkeit eine Rolle spielen könnten. So sind auch in der elterlichen Medienerziehung ${ }^{178}$ eindeutige Gruppenunterschiede zu beobachten. Auffällige Computerspieler geben bereits in der dritten Klasse deutlich seltener an, dass ihre Eltern ihre Computerspielnutzung klar reglementieren und begleiten. Ein Befund, der sich in den weiteren Jahren verfestigt.

177 USK $0=$ keine Altersbeschränkung; USK $6=$ Freigegeben ab 6 Jahren; USK $12=$ Freigegeben ab 12 Jahren; USK $16=$ Freigegeben ab 16 Jahren; USK $18=$ keine Jugendfreigabe/keine Kennzeichnung/Indizierung.

178 Summe der drei Variablen: „Bei uns gibt es klare Regelungen welche Spiele ich spielen darf und welche nicht“ (Inhalte), „Meine Eltern wollen wissen, welche Video- und Computerspiele ich spiele“ (Monitoring), „Wir haben klare zeitliche Regeln, wie lang ich Computer spielen darf" (Zeit). Skala: 3-4 Punkte $=$ kein regulatives Monitoring; 5-7 Punkte $=$ geringes regulatives Monitoring; 8-10 Punkte $=$ mittleres regulatives Monitoring; 11-12 Punkte $=$ hohes regulatives Monitoring (vgl. Kapitel 1.5.1). Dargestellt ist der Anteil der Kinder mit mittlerem oder hohem regulativen Monitoring. 
Tabelle 70. Mediennutzungsvariablen nach Computerspielabhängigkeit (5. oder 6. Klasse).

\begin{tabular}{|c|c|c|c|c|}
\hline \multirow[b]{2}{*}{ Eigener PC } & \multicolumn{2}{|c|}{$\begin{array}{c}\text { Unauffällige } \\
\text { Computerspieler } \\
(n=813)\end{array}$} & \multicolumn{2}{|c|}{$\begin{array}{l}\text { Gefährdete oder ab- } \\
\text { hängige Computerspie- } \\
\text { ler }(n=44)\end{array}$} \\
\hline & $N$ & $\%$ & $N$ & $\%$ \\
\hline 3. Klasse & 538 & $45 \%$ & 25 & $68 \% *$ \\
\hline 4. Klasse & 559 & $47 \%$ & 33 & $58 \%{ }^{\mathrm{ns}}$ \\
\hline 5. Klasse & 712 & $53 \%$ & 41 & $51 \%{ }^{\mathrm{ns}}$ \\
\hline 6. Klasse & 719 & $61 \%$ & 39 & $72 \%{ }^{\mathrm{ns}}$ \\
\hline Eigene Spielkonsole & $N$ & $\%$ & $N$ & $\%$ \\
\hline 3. Klasse & 536 & $42 \%$ & 28 & $79 \% * *$ \\
\hline 4. Klasse & 568 & $43 \%$ & 27 & $82 \% * *$ \\
\hline 5. Klasse & 717 & $57 \%$ & 41 & $73 \% *$ \\
\hline 6. Klasse & 720 & $61 \%$ & 41 & $73 \%{ }^{\mathrm{ns}}$ \\
\hline Computerspielzeiten & $N$ & $\min$. & $N$ & min. \\
\hline 3. Klasse & 550 & $33 \mathrm{~min}$. & 29 & 83 min..$^{I}$ \\
\hline 4. Klasse & 578 & $44 \mathrm{~min}$. & 33 & $131 \min . * *$ \\
\hline 5. Klasse & 739 & $39 \mathrm{~min}$. & 41 & $102 \min . * *$ \\
\hline 6. Klasse & 736 & $48 \mathrm{~min}$. & 41 & $136 \min . * *$ \\
\hline Mutter (5. Klasse) & 136 & $31 \mathrm{~min}$. & 8 & 39 min. $^{\mathrm{ns}}$ \\
\hline Vater (5. Klasse) & 137 & $51 \mathrm{~min}$. & 10 & 52 min. $^{\text {ns }}$ \\
\hline Mutter (6. Klasse) & 174 & $34 \mathrm{~min}$. & 13 & 91 min.* \\
\hline Vater (6. Klasse) & 177 & $52 \mathrm{~min}$. & 12 & 89 min. $^{\text {ns }}$ \\
\hline Genrepräferenz 5. Klasse & $N$ & $\%$ & $N$ & $\%$ \\
\hline Sportspiele & 721 & $34 \%$ & 41 & $54 \% *$ \\
\hline Party- und Mitmachspiele & 718 & $32 \%$ & 41 & $29 \%{ }^{\mathrm{ns}}$ \\
\hline $\begin{array}{l}\text { Lebens- und Aufbausimulatio- } \\
\text { nen }\end{array}$ & 718 & $31 \%$ & 41 & $24 \%{ }^{\mathrm{ns}}$ \\
\hline $\begin{array}{l}\text { Denk- und Geschicklichkeits- } \\
\text { spiele }\end{array}$ & 725 & $20 \%$ & 41 & $7 \% * *$ \\
\hline Action/Adventures & 726 & $16 \%$ & 41 & $37 \% *$ \\
\hline Onlinerollenspiele & 718 & $15 \%$ & 41 & $44 \% * *$ \\
\hline $\begin{array}{l}\text { First- und Third-Person- } \\
\text { Shooter }\end{array}$ & 719 & $14 \%$ & 41 & $54 \% * *$ \\
\hline $\begin{array}{l}\text { Strategie- und Simulations- } \\
\text { spiele }\end{array}$ & 723 & $13 \%$ & 41 & $30 \% *$ \\
\hline Prügelspiele & 715 & $9 \%$ & 41 & $44 \% * *$ \\
\hline
\end{tabular}


Unauffällige

Computerspieler

$(n=813)$
Gefährdete oder ab-

hängige Computerspieler $(n=44)$
Genrepräferenz 6. Klasse

Sportspiele

Party- und Mitmachspiele

Lebens- und Aufbausimulationen

Denk- und Geschicklichkeitsspiele

Action/Adventures

Onlinerollenspiele

First- und Third-Person-

Shooter

Strategie- und Simulationsspiele

Prügelspiele

\section{Spielmotive 5. Klasse}

Spielen bei realweltlichem Misserfolg

\section{Spielmotive 6. Klasse}

Spielen bei realweltlichem Misserfolg

\section{Computerspiele ab 16/18 Jahren}

\section{Klasse}

„Ich spiele alleine.“

„Ich spiele mit Freunden.“

„Ich spiele mit Geschwistern.“

„Ich spiele mit Vater.“

„Ich spiele mit Mutter.“

„Ich spiele mit anderen

Erwachsenen."

\section{Computerspiele ab 16/18 Jahren}

\section{Klasse}

„Ich spiele alleine.“

„Ich spiele mit Freunden.“

"Ich spiele mit Geschwistern."

„Ich spiele mit Vater.“

„Ich spiele mit Mutter.“

„Ich spiele mit anderen

Erwachsenen."
739

N

716

712

714

720

720

720

723

719

717

N

721

N

729

$\mathbf{N}$

578

578

578

578

578

578

N

739

739

739

739

739
$\%$

$32 \%$

$35 \%$

$30 \%$

$19 \%$

$16 \%$

$16 \%$

$12 \%$

$14 \%$

40

$30 \% * *$

$7 \%$

M

1.33

39

$21 \%$

$20 \%$
M

1.42

\%

N

33

33

33

33

33

33

$2 \%$

\%

N

\%

41

$41 \% *$

41

$39 \% *$

41

41

41

41
$17 \%$ ns

$2 \%$ ns

$5 \%$ ns
M

$3.18 * *$

$\%$

$49 \% * *$

$24 \%$ ns

$6 \%{ }^{\mathrm{ns}}$

$6 \%$ ns

$6 \%{ }^{\mathrm{ns}}$

$33 \%$ ns

$3 \%$ 


\title{
Computerspiele ab 16/18 Jahren
}

6. Klasse

$\begin{array}{cccc}\mathbf{N} & \mathbf{\%} & \mathbf{N} & \mathbf{\%} \\ 736 & 20 \% & 41 & 46 \%^{* *} \\ 736 & 21 \% & 41 & 46 \%^{* *} \\ 736 & 8 \% & 41 & 5 \%^{\text {ns }} \\ 736 & 3 \% & 41 & 7 \%^{\text {ns }} \\ 736 & 1 \% & 41 & 5 \%^{\text {ns }} \\ 736 & 2 \% & 41 & 5 \%^{\text {ns }}\end{array}$

„Ich spiele alleine.“
„Ich spiele mit Freunden.“
„Ich spiele mit Geschwistern."
„Ich spiele mit Vater."
„Ich spiele mit Mutter.“
„Ich spiele mit anderen
Erwachsenen."

\section{Medienerziehung Computerspiele}

3. Klasse (Anteil mittleres/ hohes regulat. Monitoring)

4. Klasse (Anteil mittleres/ hohes regulat. Monitoring) 5. Klasse (Anteil mittleres/
hohes regulat. Monitoring) 6. Klasse (Anteil mittleres/ hohes regulat. Monitoring)

\begin{tabular}{l}
557 \\
72 \\
710 \\
\hline
\end{tabular}

\section{$\mathbf{N}$}

$\%$

514

557

720

710

$\mathbf{N}$

$\mathbf{N}$

$\%$

\begin{abstract}
Anmerkung. Computerspielzeiten der Kinder wurden über die Gestern-Frage erhoben. Computerspielzeiten von Vater und Mutter sind Wochendurchschnittszeiten erhoben über den Elternfragebogen. Spielen bei realweltlichen Misserfolgen entspricht vierfach gestuftem Item: „Ich spiele häufig, wenn es in meinem Leben ansonsten gerade nicht so gut läuft" (1 - stimmt nicht, 2 - stimmt kaum, 3 - stimmt ziemlich, 4 - stimmt genau). Varianzhomogenität ist gegeben bei PC-Besitz in der vierten und fünften Klasse, bei Mediennutzungszeiten Vater/Mutter (5. Klasse). Bei allen anderen Variablen werden Signifikanzen für nicht gleiche Varianzen berichtet. Für Medienerziehung Computerspiele wurde ein Chi-Quadrat-Test, für alle anderen Variablen t-Tests für unabhängige Stichproben berechnet. * $p<.05, * * p<.01, \ddot{I} p<.10$.
\end{abstract}

\section{Risikokorrelate des Schulkontextes}

Betrachtet man Variablen des schulischen Kontexts (vgl. Tabelle 71), fällt auf, dass die Kinder, die in der fünften oder sechsten Klasse als auffällige Computerspieler eingestuft wurden, bereits in der dritten Klasse im Mittel schlechtere Noten in den Fächern Deutsch, Mathematik und Sachkunde hatten als Kinder mit einem unauffälligen Computerspielverhalten. Ein Unterschied, der sich in der vierten Klasse vergrößert und in der fünften und sechsten Klasse verfestigt. Interessanterweise ist dieser Trend aber nicht in der Intelligenzdiagnostik der Kinder zu beobachten. Zwar sind die auffälligen Computerspieler von der dritten Klasse 
an um sieben IQ-Punkte schlechter als ihre Vergleichsgruppe, in der sechsten Klasse hat sich dieser Unterschied jedoch nicht vergrößert. In diesem Licht schlechter Leistung trotz annähernd vergleichbarer kognitiver Fähigkeiten sind auch die Werte zur Schuleinstellung ${ }^{179} \mathrm{zu}$ interpretieren. Am Ende der fünften und sechsten Klasse freuen sich die Kinder mit auffälligem Computerspielverhalten weniger auf die Schule, sie stimmen der Aussage „Schule macht Spaß“ weniger zu und gehen insgesamt weniger gern zur Schule. Am Ende der sechsten Klasse spiegelt sich dies zudem in schlechteren Schullaufbahnempfehlungen wieder. Kinder, die in der fünften oder sechsten Klasse als abhängig oder gefährdet diagnostiziert wurden, bekommen im Vergleich zu ihrer Gegengruppe deutlich häufiger eine Hauptschulempfehlung und zu einem deutlich geringeren Anteil eine Gymnasialempfehlung (Gymnasium: 17\% vs. $43 \%$; Realschule: $42 \%$ vs. $44 \%$; Hauptschule: $41 \%$ vs. $13 \%$ ). Neben schlechteren schulischen Leistungen könnte ein weiterer Grund für eine negativere Schuleinstellung darin bedingt sein, dass die Kinder, die als auffällige Computerspieler identifiziert wurden, vor allem in der fünften und sechsten Klasse deutlich häufiger über eine Gewaltopfererfahrung oder Mobbing innerhalb der Schule ${ }^{180}$ innerhalb der letzten vier Wochen vor der Befragung berichten. Wiederum in Einklang mit den schlechteren schulischen Leistungen ist auch der Anteil der Kinder, die schon einmal eine Klasse wiederholen mussten, unter den auffälligen Computerspielern zwar nur zum Teil statistisch bedeutsam, aber dennoch deutlich (um mehr als das Doppelte) erhöht. Entsprechend ist auch die Quote der Schulschwänzer, also von Kindern die innerhalb der letzten 12 Monate einen ganzen Tag oder nur einzelne Stunden die Schule geschwänzt haben, vor allem in der sechsten Klasse unter den auffälligen Computerspielern deutlich erhöht.

179 Mittelwerte der drei viertstufigen Items: „Morgens freue ich mich auf die Schule“, „Schule macht Spaß“, „Ich gehe gern zur Schule“. Hohe Werte auf dieser Skala stehen für Freude und Spaß an der Schule (Itemkennwerte siehe Kapitel 1.5.1, Tabelle 17).

180 Ein Schüler oder eine Schülerin wurde als Opfer von Gewalt in der Schule eingestuft, wenn ihm innerhalb der letzten vier Wochen vor der Befragung eines der folgenden Dinge mindestens einmal passiert ist: „Ich wurde Schülern geschlagen oder getreten, und zwar nicht aus Spaß", ,Schüler haben mit Absicht meine Sachen kaputtgemacht“, ,Schüler haben mich gezwungen, etwas zu tun, was ich nicht wollte“. Ein Schüler oder eine Schülerin wurde als Opfer von Mobbing in der Schule eingestuft, wenn ihm innerhalb der letzten vier Wochen vor der Befragung eines der folgenden Dinge mindestens einmal passiert ist: „Schüler haben mich gehänselt oder hässliche Dinge über mich gesagt", „Andere Schüler haben mich wie Luft behandelt und absichtlich nicht mehr beachtet", "Andere Schüler haben Gerüchte über mich verbreitet, die nicht wahr gewesen sind", „Ich durfte in der Pause nicht bei anderen Kindern mitspielen". 
Tabelle 71. Leistungsvariablen und Variablen des Schulkontextes nach Computerspielabhängigkeit (5. oder 6. Klasse).

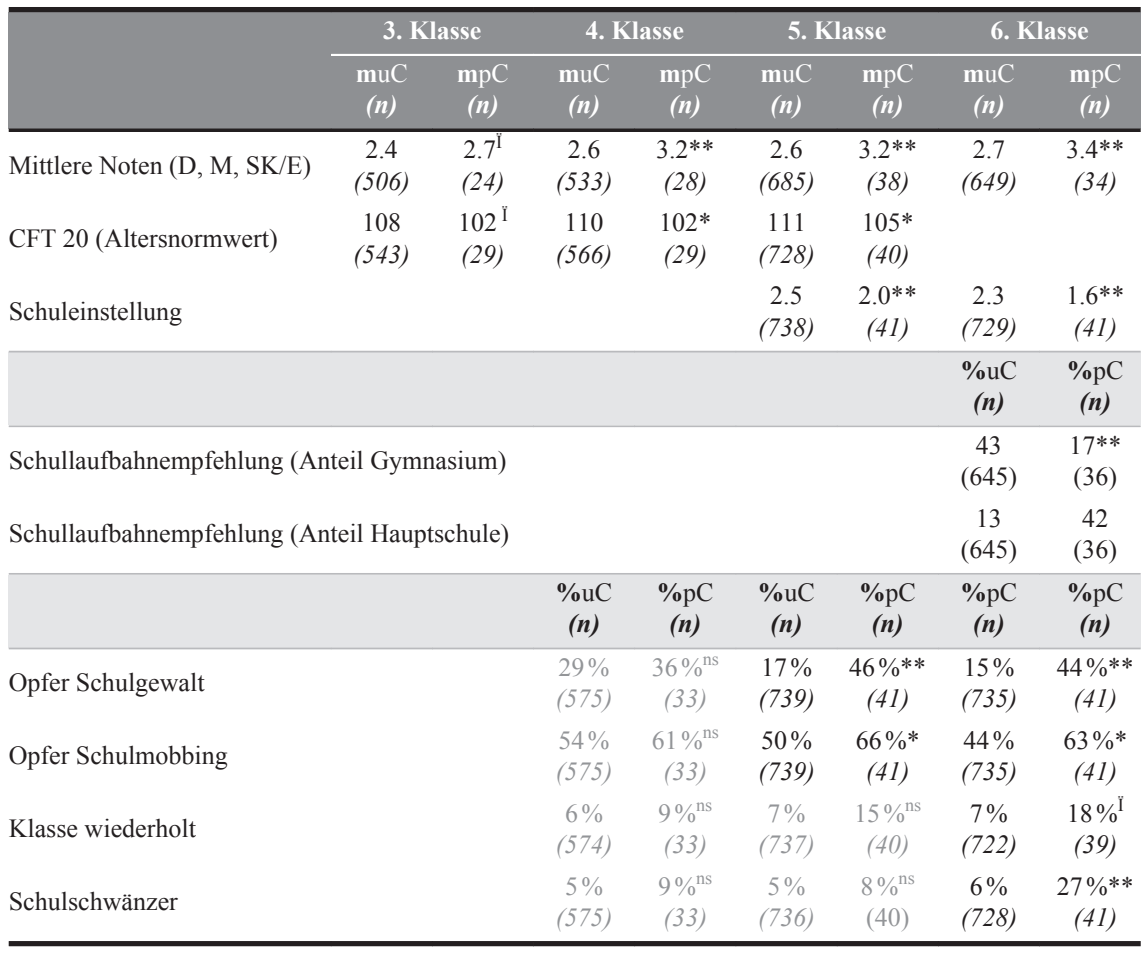

Anmerkung. $\mathrm{m}_{\mathrm{uC}} / \mathrm{m}_{\mathrm{uC}}=$ Mittelwert/Anteil Prozent unauffällige Computerspieler, $\mathrm{m}_{\mathrm{pC}} / \mathrm{m}_{\mathrm{pC}}=$ Mittelwert/Anteil Prozent gefährdeter bzw. abhängiger Computerspieler. In Klammern ist jeweils die zur Berechnung zugrunde liegende Stichprobenanzahl angegeben. Signifikanzen für nicht gleiche Varianzen berichtet für: Opfer Schulgewalt, Klasse wiederholt, Schulschwänzen. Für Schullaufbahnempfehlung wurde ein Chi-Quadrat-Test, für alle anderen Variablen t-Tests für unabhängige Stichproben berechnet. * $p<.05, * * p<.01, \ddot{I} p<.10$.

\section{Risikokorrelate (bzw. Komorbiditäten) der Persönlichkeit}

An der Schnittstelle zwischen Schulkontext und Persönlichkeitsvariablen können die beiden Konstrukte Soziale Integration ${ }^{181}$ und Selbstkonzept eigener

181 Skala soziale Integration: Summenscore der vierstufigen (,stimmt nicht“, „stimmt kaum“, ,stimmt ziemlich“, ,stimmt genau“) Items: „Meine Mitschüler sind nett zu mir“, „Ich komme mit anderen Kindern in meiner Klasse gut aus“, „Ich habe wenig Freunde in meiner Klasse“ (umgepolt). Hohe Werte auf dieser Skala stehen für eine gute Integration in die Klassengemeinschaft (siehe Kapitel 1.5.2, Tabelle 22). 
Schulfähigkeiten ${ }^{182}$ betrachtet werden (vgl. Tabelle 72). Auch hier sind z. T. auffällige Werte bei den gefährdeten oder abhängigen Computerspielern zu beobachten. So empfanden sich diese in der dritten Klasse im Mittel schlechter in den Klassenverbund sozial integriert als die Vergleichsgruppe der unauffälligen Computerspieler. Ein Unterschied, der sich auch in den folgenden drei Jahren so deutlich darstellt. Ebenso vergrößert sich der Unterschied zwischen den beiden Gruppen in der Einschätzung ihre schulischen Fähigkeiten (geringeres Selbstkonzept eigener Schulfähigkeiten bei auffälligen Computerspielern) von der dritten bis zur sechsten Klasse, ein Trend, der dem der Schulnoten entspricht. Hin-

Tabelle 72. Persönlichkeitsvariablen nach Jahrgangsstufe und Computerspielabhängigkeit (5. bzw. 6. Klasse).

\begin{tabular}{|c|c|c|c|c|c|c|c|c|}
\hline & \multicolumn{2}{|c|}{ 3. Klasse } & \multicolumn{2}{|c|}{ 4. Klasse } & \multicolumn{2}{|c|}{ 5. Klasse } & \multicolumn{2}{|c|}{ 6. Klasse } \\
\hline & $\begin{array}{c}\mathrm{muC} \\
(n)\end{array}$ & $\begin{array}{c}\mathrm{mpC} \\
(n)\end{array}$ & $\begin{array}{c}\mathrm{muC} \\
(n)\end{array}$ & $\begin{array}{c}\mathrm{mpC} \\
(\text { (n) }\end{array}$ & $\begin{array}{c}\mathrm{muC} \\
(n)\end{array}$ & $\begin{array}{c}\mathrm{mpC} \\
(n)\end{array}$ & $\begin{array}{c}\mathrm{muC} \\
(n)\end{array}$ & $\begin{array}{c}\mathrm{mpC} \\
(n)\end{array}$ \\
\hline Soziale Integration & $\begin{array}{c}49 \\
(540)\end{array}$ & $\begin{array}{l}46^{1} \\
(28)\end{array}$ & $\begin{array}{c}50 \\
(576)\end{array}$ & $\begin{array}{l}46^{*} \\
(33)\end{array}$ & $\begin{array}{c}3.3 \\
(740)\end{array}$ & $\begin{array}{c}2.9^{* *} \\
(41)\end{array}$ & $\begin{array}{c}3.4 \\
(730)\end{array}$ & $\begin{array}{c}2.9^{* *} \\
(41)\end{array}$ \\
\hline $\begin{array}{l}\text { Selbstkonzept eigener Schul- } \\
\text { fähigkeit }\end{array}$ & $\begin{array}{c}49 \\
(540)\end{array}$ & $\begin{array}{l}45^{*} \\
(28)\end{array}$ & $\begin{array}{c}51 \\
(576)\end{array}$ & $\begin{array}{r}42 * * \\
(33)\end{array}$ & $\begin{array}{c}3.1 \\
(740)\end{array}$ & $\begin{array}{c}2.4^{* *} \\
(41)\end{array}$ & $\begin{array}{c}3.1 \\
(730)\end{array}$ & $\begin{array}{c}2.4^{* *} \\
(41)\end{array}$ \\
\hline Depressive Verstimmung & $\begin{array}{l}9.5 \\
(547)\end{array}$ & $\begin{array}{c}10.6^{\mathrm{I}} \\
(28)\end{array}$ & $\begin{array}{l}9.6 \\
(574)\end{array}$ & $\begin{array}{c}10.8^{*} \\
(33)\end{array}$ & $\begin{array}{c}9.6 \\
(732)\end{array}$ & $\begin{array}{c}10.7^{*} \\
(41)\end{array}$ & $\begin{array}{l}10.1 \\
(724)\end{array}$ & $\begin{array}{c}12.4 * * \\
(39)\end{array}$ \\
\hline Empathie (IVE) & & & $\begin{array}{c}3.1 \\
(575)\end{array}$ & $\begin{array}{l}2.7^{\mathrm{ns}} \\
(32)\end{array}$ & $\begin{array}{c}3.1 \\
(737)\end{array}$ & $\begin{array}{l}2.4^{*} \\
(41)\end{array}$ & $\begin{array}{c}3.0 \\
(733)\end{array}$ & $\begin{array}{c}1.9^{* *} \\
(41)\end{array}$ \\
\hline \multirow[t]{2}{*}{ Impulsivität (IVE) } & & & $\begin{array}{c}1.1 \\
(575)\end{array}$ & $\begin{array}{c}1.9^{* *} \\
(32)\end{array}$ & $\begin{array}{c}1.1 \\
(738)\end{array}$ & $\begin{array}{c}2.3^{* *} \\
(41)\end{array}$ & $\begin{array}{c}1.1 \\
(735)\end{array}$ & $\begin{array}{c}2.6^{* *} \\
(41)\end{array}$ \\
\hline & & & & & $\begin{array}{c}\% \mathrm{uC} \\
(n)\end{array}$ & $\begin{array}{l}\% \mathrm{pC} \\
(n)\end{array}$ & $\begin{array}{c}\% \text { uC } \\
\text { (n) }\end{array}$ & $\begin{array}{l}\% \mathrm{pC} \\
\text { (n) }\end{array}$ \\
\hline \multicolumn{3}{|c|}{ SDQ Hyperaktivität (Anteil auffällig) } & & & $\begin{array}{l}11 \% \\
(724)\end{array}$ & $\begin{array}{c}34 \% * * \\
(41)\end{array}$ & $\begin{array}{l}7 \% \\
(704)\end{array}$ & $\begin{array}{l}39 \% * * \\
(41)\end{array}$ \\
\hline \multicolumn{3}{|c|}{$\begin{array}{l}\text { SDQ prosoziales Verhalten (Anteil } \\
\text { auffällig) }\end{array}$} & & & $\begin{array}{c}4 \% \\
(725)\end{array}$ & $\begin{array}{c}23 \% * * \\
(40)\end{array}$ & $\begin{array}{l}4 \% \\
(713)\end{array}$ & $\begin{array}{c}24 \% * * \\
(41)\end{array}$ \\
\hline \multicolumn{3}{|c|}{$\begin{array}{l}\text { SDQ Verhaltensprobleme (Anteil auf- } \\
\text { fällig) }\end{array}$} & & & $\begin{array}{l}6 \% \\
(721)\end{array}$ & $\begin{array}{c}21 \% * * \\
(39)\end{array}$ & $\begin{array}{l}6 \% \\
(703)\end{array}$ & $\begin{array}{c}28 \% * * \\
(39)\end{array}$ \\
\hline
\end{tabular}

Anmerkung. $\mathrm{m}_{\mathrm{uC}} / \mathrm{m}_{\mathrm{uC}}=$ Mittelwert/Anteil Prozent unauffällige Computerspieler, $\mathrm{m}_{\mathrm{pC}} / \mathrm{m}_{\mathrm{pC}}=$ Mittelwert/Anteil Prozent gefährdeter bzw. abhängiger Computerspieler. In Klammern ist jeweils die zur Berechnung zugrunde liegende Stichprobenanzahl angegeben. Signifikanzen für nicht gleiche Varianzen berichtet für: Soziale Integration (5. und 6. Klasse), Selbstkonzept eigener Fähigkeiten (6. Klasse), Depressive Verstimmung (5. und 6. Klasse), Empathie. Für den SDQ wurde ein Chi-Quadrat-Test, für alle anderen Variablen t-Tests für unabhängige Stichproben berechnet. $* p<.05, * * p<$. $01, \ddot{\mathrm{I}} p<.10$.

182 Skala Selbstkonzept eigener Schulfähigkeiten: Summenscore der vierstufigen (,stimmt nicht“, „stimmt kaum“, „stimmt ziemlich“, ,stimmt genau“) Items: „Ich lerne sehr langsam (umgepolt)“, „Ich mache in der Schule das meiste richtig“, „Ich kann ganz gut lernen“, „Ich bin gut in der Schule“. Hohe Werte auf dieser Skala stehen für eine hohe Einschätzung ihrer schulischen Fähigkeiten (siehe Kapitel 1.5.2, Tabelle 21). 
sichtlich einer depressiven Problematik ${ }^{183}$ erreichen zu allen Messzeitpunkten Kinder mit einem auffälligen Computerspielverhalten höhere Werte auf dieser Skala, was einer höheren depressiven Verstimmung entspricht.

Als zwei weitere Persönlichkeitseigenschaften, die im Zusammenhang mit auffälligem Computerspielverhalten berichtet werden, wurden Empathie ${ }^{184}$ und Impulsivität ${ }^{185}$ mithilfe des IVE (Stadler et al., 2004a) erfasst. Wie für Kinder beobachtet, die extrem gewalthaltige Computerspiele bevorzugen, können auch für computerspielabhängige Kinder bedeutsame Unterschiede hinsichtlich ihrer empathischen Fähigkeiten festgestellt werden. Diese liegen zu allen Messzeitpunkten auf einem geringeren Niveau, wobei dieser Unterschied allerdings nur in der fünften und sechsten Klasse statistische Signifikanz erreicht. Ebenso sind von der vierten bis zur sechsten Klasse deutliche Unterschiede hinsichtlich der gemessenen Impulsivitätswerte zu verzeichnen. Auffällige Computerspieler haben demnach einen schnellen und ungenauen Arbeitsstil, eine mangelnde Voraussicht eigener Handlungskonsequenzen, orientieren sich vor allem an sofort verfügbaren positiven Konsequenzen eigener Handlungen und richten diese kaum an zukünftigen Zielen aus. Über die Zeit scheint sich dieser Unterschied zudem zu vergrößern.

Als eine weitere mögliche Komorbidität einer Computerspielabhängigkeit wurde schließlich, neben der depressiven Verstimmung, Hyperaktivität mithilfe des Strengths and Difficulties Questionnaire in der deutschen Übersetzung (SDQ-Deu; Goodman, 1997; Klasen et al., 2000) im Fragebogen der fünften und sechsten Klasse erhoben. ${ }^{186}$ Des Weiteren kamen auch die Skalen ,prosoziales Verhalten“ und „Verhaltensprobleme mit Gleichaltrigen“ desselben Instrumentes zur Verwendung. Ein problematisches Verhalten, welches durch die Skala Impulsivität schon angedeutet wurde, wird durch die bezüglich Hyperaktivität gemessenen Werte deutlich bestätigt. So liegt der Anteil der hyperaktiv Auffälligen bei gefährdeten oder abhängigen Computerspielern bei 34 bzw. 39 Prozent (unauffällige Computerspieler: $11 \%$ bzw. $7 \%$ ). Ferner zeigen Computerspielabhängige in der fünften und sechsten Klasse auch häufiger ein prosozial auffälliges Verhalten, dahin gehend, dass sie weniger nett und hilfsbereit sind und anderen weniger helfen. Da das Konstrukt „Verhaltensprobleme mit Gleichaltrigen“ einen großen Überschneidungsbereich mit dem Konstrukt „soziale Integration“ aufweist, ist es nicht verwunderlich, dass hier in der ein deutlich höherer Anteil der computerspielabhängigen Kinder auffällig wird.

183 Vgl. Kapitel 1.5.1, siehe Tabelle 18.

184 Vgl. Kapitel 1.5.2, siehe Tabelle 24.

185 Vgl. Kapitel 1.5.2, siehe Tabelle 23.

186 Vgl. Kapitel 1.5.2. 
Hinsichtlich der sozialen Variablen (vgl. Tabelle 73) lassen sich die Befunde für die vorliegende Stichprobe wie folgt zusammenfassen: Kinder, die Computerspiele in einer auffälligen Weise nutzen, sind deutlich häufiger Scheidungskinder, berichten häufiger über geringere elterliche Zuwendung ${ }^{187}$ und erleben häufiger Gewalt im Elternhaus, ${ }^{188}$ werden also von ihren Eltern geschlagen oder gar verprügelt. Des Weiteren kommen diese Kinder öfter aus einem Elternhaus mit niedrigem Bildungsniveau (beide Eltern unterhalb Realschulabschluss) und geringem Wohlstand. Als letztes Unterscheidungsmerkmal sind auffällige Computerspieler der sechsten Klasse schließlich primär (deutsche) Jungen.

Tabelle 73. Variablen der Sozialstruktur nach Jahrgangsstufe und Computerspielabhängigkeit (5. bzw. 6. Klasse).

\begin{tabular}{|c|c|c|c|c|c|c|c|c|}
\hline & \multicolumn{2}{|c|}{ 3. Klasse } & \multicolumn{2}{|c|}{ 4. Klasse } & \multicolumn{2}{|c|}{ 5. Klasse } & \multicolumn{2}{|c|}{ 6. Klasse } \\
\hline & $\begin{array}{c}\% \mathrm{uC} \\
(n)\end{array}$ & $\begin{array}{c}\% \mathrm{pC} \\
(n)\end{array}$ & $\begin{array}{c}\% \mathrm{uC} \\
(n)\end{array}$ & $\begin{array}{c}\% \mathrm{pC} \\
(n)\end{array}$ & $\begin{array}{c}\% \mathrm{uC} \\
(n)\end{array}$ & $\begin{array}{c}\% \mathrm{pC} \\
(n)\end{array}$ & $\begin{array}{c}\% \mathrm{uC} \\
(n)\end{array}$ & $\begin{array}{c}\% \mathrm{pC} \\
(n)\end{array}$ \\
\hline Scheidungskinder & & & $\begin{array}{l}25 \% \\
(566)\end{array}$ & $\begin{array}{c}40 \%{ }^{\mathrm{ns}} \\
(33)\end{array}$ & $\begin{array}{l}28 \% \\
(720)\end{array}$ & $\begin{array}{c}39 \%^{\mathrm{ns}} \\
(36)\end{array}$ & $\begin{array}{l}26 \% \\
(720)\end{array}$ & $\begin{array}{c}44 \% * \\
(39)\end{array}$ \\
\hline Erlebte Elterngewalt & & & $\begin{array}{l}20 \% \\
(577)\end{array}$ & $\begin{array}{c}33 \%{ }^{\mathrm{ns}} \\
(33)\end{array}$ & $\begin{array}{l}15 \% \\
(737)\end{array}$ & $\begin{array}{c}34 \% * \\
(41)\end{array}$ & $\begin{array}{l}14 \% \\
(736)\end{array}$ & $\begin{array}{c}37 \% * * \\
(41)\end{array}$ \\
\hline $\begin{array}{l}\text { Anteil geringe elterliche Zuwen- } \\
\text { dung }\end{array}$ & $\begin{array}{l}2 \% \\
(510)\end{array}$ & $\begin{array}{l}7 \% * \\
(27)\end{array}$ & $\begin{array}{l}3 \% \\
(535)\end{array}$ & $\begin{array}{l}7 \% * \\
(30)\end{array}$ & $\begin{array}{l}2 \% \\
(700)\end{array}$ & $\begin{array}{c}8 \% * * \\
(38)\end{array}$ & $\begin{array}{c}4 \% \\
(683)\end{array}$ & $\begin{array}{c}18 \% * * \\
(38)\end{array}$ \\
\hline Anteil hohe elterliche Zuwendung & $\begin{array}{l}65 \% \\
(510)\end{array}$ & $\begin{array}{l}41 \% \\
(27)\end{array}$ & $\begin{array}{l}69 \% \\
(535)\end{array}$ & $\begin{array}{l}47 \% \\
(30)\end{array}$ & $\begin{array}{l}68 \% \\
(700)\end{array}$ & $\begin{array}{c}37 \% \\
(38)\end{array}$ & $\begin{array}{l}62 \% \\
(683)\end{array}$ & $\begin{array}{l}29 \% \\
(38)\end{array}$ \\
\hline $\begin{array}{l}\text { Anteil geringes elterliches Bil- } \\
\text { dungsniveau }\end{array}$ & & & & & & & $\begin{array}{l}12 \% \\
(724)\end{array}$ & $\begin{array}{c}28 \% * * \\
(36)\end{array}$ \\
\hline $\begin{array}{l}\text { Anteil hohes elterliches Bildungs- } \\
\text { niveau }\end{array}$ & & & & & & & $\begin{array}{l}53 \% \\
(724)\end{array}$ & $\begin{array}{l}25 \% \\
(36)\end{array}$ \\
\hline $\begin{array}{l}\text { Anteil geringer Wohlstand - Q1, } \\
\text { Q2 }\end{array}$ & & & & & & & $\begin{array}{l}41 \% \\
(813)\end{array}$ & $\begin{array}{c}52 \% * \\
(44)\end{array}$ \\
\hline Anteil hoher Wohlstand - Q4, Q5 & & & & & & & $\begin{array}{l}39 \% \\
(813)\end{array}$ & $\begin{array}{l}21 \% \\
(44)\end{array}$ \\
\hline $\begin{array}{l}\text { Anteil Kinder mit Migrationshin- } \\
\text { tergrund }\end{array}$ & & & & & & & $\begin{array}{l}29 \% \\
(813)\end{array}$ & $\begin{array}{l}23 \%{ }^{\mathrm{ns}} \\
(44)\end{array}$ \\
\hline
\end{tabular}

Anmerkung. $\mathrm{m}_{\mathrm{uC}} / \mathrm{m}_{\mathrm{uC}}=$ Mittelwert/Anteil Prozent unauffällige Computerspieler, $\mathrm{m}_{\mathrm{pC}} / \mathrm{m}_{\mathrm{pC}}=$ Mittelwert/Anteil Prozent gefährdeter bzw. abhängiger Computerspieler. In Klammern ist jeweils die zur Berechnung zugrunde liegende Stichprobenanzahl angegeben. Signifikanzen für nicht gleiche Varianzen berichtet für: Scheidungskinder, Elterngewalt. Für elterliche Zuwendung, Bildung, Wohlstand und Migrationshintergrund wurde ein Chi-Quadrat-Test, für alle anderen Variablen t-Tests für unabhängige Stichproben berechnet. * $p<.05, * * p<.01$, Ï $p<.10$. 


\subsubsection{Problematisches Computerspielen im Kindesalter - ein Risikomodell}

Um die gefundenen Zusammenhänge zwischen Computerspielabhängigkeit und Faktoren der Mediennutzung, der Persönlichkeit und des sozialen sowie schulischen Umfeldes (samt ihrer Wechselwirkungen) multivariat betrachten zu können, wurde ein Strukturgleichungsmodell berechnet, welches jene Variablen beinhaltet, die sich in den bivariaten Analysen als bedeutsam erwiesen haben (vgl. Abbildung 74). Soziale Integration und Impulsivität wurden wegen ihrer großen inhaltlichen Übereinstimmung mit den Konstrukten Verhaltensprobleme mit Gleichaltrigen respektive Hyperaktivität des Strengths and Difficulties Questionnaire nicht extra in diesem Modell abgebildet. Die Erfassung der Persönlichkeitsvariablen mittels eines Testverfahrens wurde hier vorgezogen. Da die Fallzahl der computerspielabhängigen bzw. gefährdeten Spieler mit $n=44$ in der fünften und sechsten Klasse zur Berechnung eines Modells jedoch als zu gering erschien, ging als abhängige Variable die Summenvariable zur Computerspielabhängigkeit (in der sechsten Klasse) ein. Diese kann auf einem Kontinuum von unproblematischem hin zu problematischem Computerspielverhalten als Maß für die Tendenz, dass eine befragte Person für abhängiges Computerspielen anfällig ist, betrachtet werden.

In der gewählten Modellstruktur zeigt sich bei guter Passungsgüte des Modells $(\chi 2=38.59(28, N=724), R M S E A=.02, R M R=.13, S R M R=.03, G F I=.99$, $A G F I=.98)$ und einer Varianzaufklärung von 56 Prozent, bei gleichzeitiger Kontrolle weiterer Faktoren, zunächst eine eigenständige Erklärungskraft bestimmter Computerspielnutzungsmuster für die Ausprägung eines problematischen Computerspielverhaltens (wenn diese auch hinter bedeutsameren Persönlichkeitsfaktoren zurückstehen). So kann die häufige Nutzung von Onlinerollenspielen ( $\beta=$. $08)$ sowie die häufige Nutzung von First- und Third-Person-Shootern ${ }^{189}(\beta=.11)$, beides Genres die vor allem von Jungen präferiert werden (First- und Third-Person-Shooter: $\beta=.40$; Onlinerollenspiele: $\beta=.24$ ), bei gleichzeitiger Berücksichtigung des Geschlechts (Jungen zeigen unabhängig ihrer Genrepräferenz ein problematischeres Computerspielverhalten, $\beta=.07$ ), zu einem gewissen Anteil problematisches Computerspielverhalten erklären. Eine deutlich höhere Erklärungskraft (die größte im gesamten Modell) hat das Computerspielen bei realweltlichen Misserfolgen $(\beta=.53)$. Je stärker die Kinder zustimmen, Computerspiele insbesondere dann zu nutzen, wenn es in ihrem Leben gerade ,nicht so gut läuft", - ein Verhalten das deutlich häufiger auf Jungen zutrifft $(\beta=.19)$ - desto höher fällt ihr Risiko für eine Computerspielabhängigkeit aus. Dieser Prädiktor tangiert das Konstrukt der dysfunktionalen Stressregulation, bei welcher das 
Spielen stellvertretend für eine Auseinandersetzung mit realweltlichen Problemen oder Konflikten stattfindet. Ist dies der Fall, werden gehäuft Onlinerollenspiele $(\beta=.26)$ Third- und First-Person-Shooter gespielt $(\beta=.24)$.

Im Bereich der Persönlichkeitsvariablen haben neben einem niedrigen Selbstkonzept eigener Schulfähigkeiten $(\beta=.07)$, vor allem Hyperaktivität $(\beta=.11)$ und Verhaltensprobleme mit Gleichaltrigen $(\beta=.11)$ eine eigenständige Erklärungskraft für problematisches Computerspielverhalten. Ersteres wiederum ein Bereich, in dem vor allem Jungen auffällig werden $(\beta=.09)$. Das Vorhandensein einer depressiven Verstimmung, welches öfters von Mädchen berichtet wird $(\beta=-$. 10) kann problematisches Computerspielen jedoch nicht vorhersagen $\left(\beta=.01^{\text {ns }}\right)$. Ein auffälliges hyperaktives Verhalten $(\beta=.12)$, berichtete Probleme mit Gleichaltrigen $(\beta=.15)$, sowie eine depressive Verstimmung $(\beta=.10)$ führen des Weiteren dazu, dass Computerspiele insbesondere dann genutzt werden, wenn es im Leben gerade „nicht so gut läuft“ - Computerspielen bei realweltlichen Misserfolgen.

Ein weiterer Einflussfaktor für die Entstehung einer Computerspielabhängigkeit ist das soziale Umfeld der Kinder. Geben die Kinder an, wenig Zuwendung von ihren Eltern zu erfahren und berichten die Kinder zusätzlich über häufige und schwere Elterngewalt in der Kindheit, hat dies zwar jeweils keine eigenständige Erklärungskraft für problematisches Computerspielverhalten $\left(\beta=.04^{\mathrm{ns}}\right.$ bzw. $\left.\beta=.03^{\text {ns }}\right)$. Beides kann aber wegen der Zusammenhänge mit den oben berichteten Persönlichkeitsvariablen als moderierender Hintergrundfaktor betrachtet werden ( $\varphi=.13$ bis $\varphi=.32$ ). Von besonderer Bedeutung sind schließlich die Zusammenhänge der Variablen des sozialen Umfeldes mit Computerspielen bei realweltlichen Misserfolgen. Erleben Kinder Gewalt im Elternhaus $(\beta=.11)$ und erfahren insgesamt wenig Zuneigung von ihren Eltern $(\beta=.15)$, führt das häufiger dazu das Medium Computerspiel zu nutzen, wenn es Probleme im realen Leben gibt. 


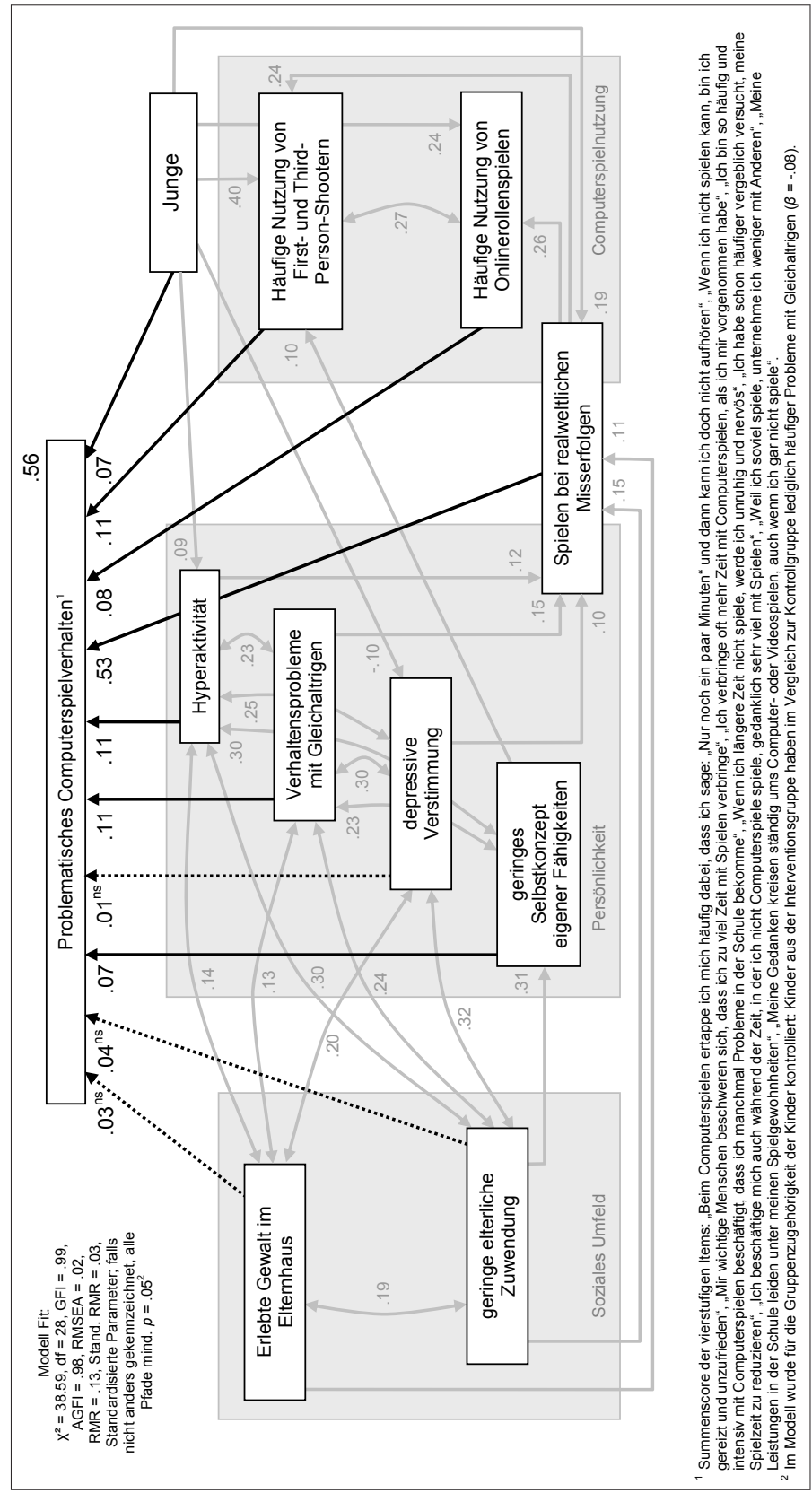

Abbildung 74. Strukturgleichungsmodell zur Erklärung von problematischem Computerspielverhalten (6. Klasse). 
Die Querschnittsbefunde aus Tabelle 71, Tabelle 72, Tabelle 73 sowie Abbildung 74 zeigen zur Erklärung problematischen Computerspielverhaltens und einer möglichen Computerspielabhängigkeit ein komplexes Zusammenspiel aus Variablen der Persönlichkeit (Hyperaktivität, Verhaltensprobleme mit Gleichaltrigen, geringes Selbstkonzept eigener Schulfähigkeiten), Variablen des sozialen Umfeldes (geringe elterliche Zuwendung und erlebte Elterngewalt) sowie Variablen der Computerspielnutzung (Spielen bei realweltlichen Misserfolgen, häufige Nutzung von Onlinerollenspielen und First- und Third-Person-Shootern). Ferner zeigten die bisherigen Auswertungen dass Kinder, die sich in der fünften oder sechsten Klasse durch ein problematisches Computerspielverhalten kennzeichnen, auch schon besonders früh, d. h. bereits in der dritten Klasse, eine auffällige psychosoziale Belastung hatten (vgl. Tabelle 71, Tabelle 72, Tabelle 73). Ziel der weiteren Ausführungen soll es sein, anhand der Längsschnittdaten erstens empirisch begründete Aussagen über die genaue Richtung und Ursächlichkeit der jeweiligen Zusammenhänge mit problematischem Computerspielverhalten zu machen und zweitens Variablen zu identifizieren, die schon besonders früh (und auch besonders gut) ein problematisches Computerspielverhalten vorhersagen können.

Zunächst wurden einzelne Strukturgleichungsmodelle für die verschiedenen Variablen der Persönlichkeit, des sozialen Umfelds und der Computerspielnutzung berechnet (d. h. depressive Verstimmung, ${ }^{190}$ Hyperaktivität, ${ }^{191}$ Verhaltensprobleme mit Gleichaltrigen, ${ }^{192}$ Selbstkonzept eigener Fähigkeiten, ${ }^{193}$ Spielen bei realweltlichen Misserfolgen ${ }^{194}$ sowie Nutzung von Onlinerollenspielen ${ }^{195}$ und First- und Third-Person-Shootern), ${ }^{196}$ jeweils im Bezug zu problematischem Computerspielverhalten und jeweils für die beiden Messzeitpunkte, zu denen in beiden Bereichen Befragungsdaten vorlagen (fünfte und sechste Klasse, siehe Abbildung 75 und Abbildung 76). Problematisches Computerspielverhalten wur-

190 Vgl. Kapitel 1.5.1, Tabelle 18.

191 SDQ-Hyperaktivität, vgl. Kapitel 1.5.2.

192 SDQ-Verhaltensprobleme mit Gleichaltrigen, vgl. Kapitel 1.5.2.

193 Selbstkonzept eigener Schulfähigkeit, siehe Kapitel 1.5.2, Tabelle 21.

194 Spielen bei realweltlichen Misserfolgen entspricht vierfach gestuftem Item: „Ich spiele häufig, wenn es in meinem Leben ansonsten gerade nicht so gut läuft" (1 - stimmt nicht, 2 - stimmt kaum, 3- stimmt ziemlich, 4 - stimmt genau).

195 Vgl. Kapitel 2.2.3.

196 Häufige Nutzung von First- und Third-Person-Shootern, vgl. Kapitel 2.2.3. 
de analog zum vorherigen Kapitel 5.2.4 über die Summenvariable zur Computerspielabhängigkeit operationalisiert. ${ }^{197}$

197 Aus Gründen der Vergleichbarkeit der Daten der fünften und sechsten Klasse gingen allerdings in die Summenbildung nur die zehn Variablen ein, die zu beiden Messzeitpunkten abgefragt wurden: „Ich beschäftige mich auch während der Zeit, in der ich nicht Computerspiele spiel, gedanklich sehr viel mit Spielen.“, „Meine Gedanken kreisen ständig ums Computer- und Videospielen, auch wenn ich gar nicht spiele.“, „Meine Leistungen in der Schule leiden unter meinen Spielgewohnheiten.“, „Ich bin so häufig und intensiv mit Computer- und Videospielen beschäftigt, dass ich manchmal Probleme in der Schule bekomme.“, „Mir wichtige Menschen beschweren sich, dass ich zu viel Zeit mit Spielen verbringe.“, „Weil ich soviel spiele, unternehme ich weniger mit anderen.“, „Ich verbringe oft mehr Zeit mit Computer- und Videospielen, als ich mir vorgenommen habe.“, „Ich habe das Gefühl, meine Spielzeit nicht kontrollieren zu können.“, ,Wenn ich nicht spielen kann, bin ich gereizt und unzufrieden.“, „Wenn ich längere Zeit nicht spiele, werde ich unruhig und nervös.“. Da das Item „Beim Computerspielen ertappe ich mich häufig dabei, dass ich sage: ,Nur noch ein paar Minuten' und dann kann ich doch nicht aufhören." in der sechsten Klasse nicht abgefragt wurde, konnte die KFN-CSAS-I nicht eins zu eins abgebildet werden. Die neugebildete Skala hat somit einen Wertebereich von 10 bis 40 Punkten und korreliert mit der KFN-CSAS-I sowie mit der KFN-CSAS-II mit $r$ $=.99$. 


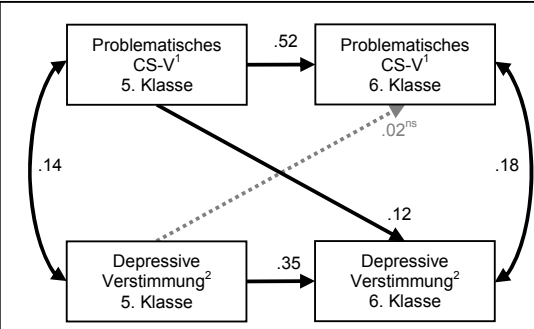

Modell Fit:

$\mathrm{X}^{2}=.03 \mathrm{df}=2, \mathrm{TLI}=1, \mathrm{CFI}=1, \mathrm{RMSEA}=.00$, Standardisierte Parameter; alle Pfade mindestens $p \leq .05$ (falls nicht anders gekennzeichnet). ${ }^{8}$

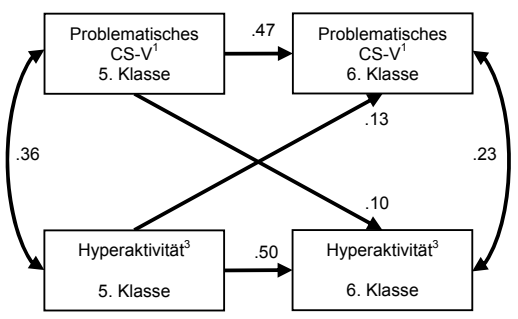

Modell Fit:

$\mathrm{X}^{2}=1.4 \mathrm{df}=2, \mathrm{TLI}=1, \mathrm{CFI}=1, \mathrm{RMSEA}=.00$, Standardisierte Parameter; alle Pfade mindestens $p \leq .05$ (falls nicht anders gekennzeichnet). ${ }^{8}$

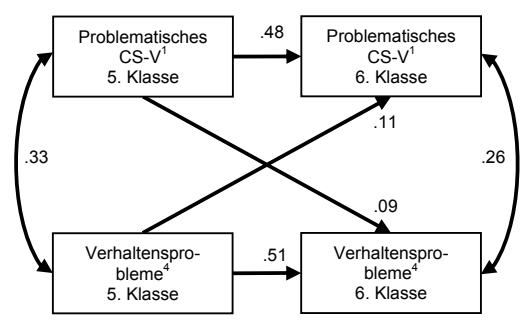

Modell Fit:

$\mathrm{X}^{2}=3.2 \mathrm{df}=2, \mathrm{TLI}=.98, \mathrm{CFI}=.99, \mathrm{RMSEA}=.03$, Standardisierte Parameter; alle Pfade mindestens $p \leq .05$ (falls nicht anders gekennzeichnet). ${ }^{8}$

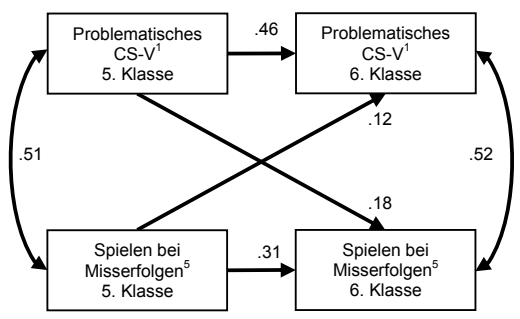

Modell Fit:

$\mathrm{X}^{2}=.02 \mathrm{df}=2, \mathrm{TLI}=1, \mathrm{CFI}=1, \mathrm{RMSEA}=.00$, Standardisierte Parameter; alle Pfade mindestens $p \leq .05$ (falls nicht anders gekennzeichnet). ${ }^{8}$

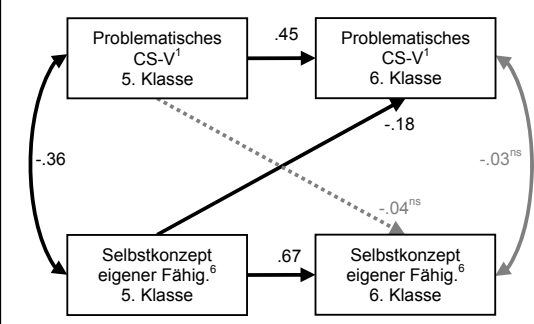

Modell Fit:

$\mathrm{X}^{2}=3.6 \mathrm{df}=2, \mathrm{TLI}=.99, \mathrm{CFI}=.99, \mathrm{RMSEA}=.03$, Standardisierte Parameter; alle Pfade mindestens $p \leq .05$ (falls nicht anders gekennzeichnet). ${ }^{8}$

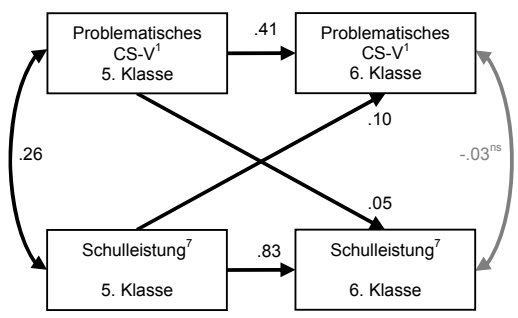

Modell Fit:

$\mathrm{X}^{2}=.09 \mathrm{df}=2, \mathrm{TLI}=1, \mathrm{CFI}=1, \mathrm{RMSEA}=.00$, Standardisierte Parameter; alle Pfade mindestens $p \leq .05$ (falls nicht anders gekennzeichnet). ${ }^{8}$

${ }^{1}$ Problematisches Computerspielverhalten, vgl. Kapitel 1.5.1

${ }^{3}$ SDQ-Hyperaktivität, vgl. Kapitel 1.5.2

2 vgl. Kapitel 1.5.1, Tabelle 18

${ }^{4}$ SDQ-Verhaltensprobleme mit Gleichaltrigen, vgl. Kapitel 1.5.2

5 Ich spiele häufig, wenn es in meinem Leben ansonsten gerade

nicht so gut läuft

${ }^{7}$ Mittlere Schulnoten in Deutsch, Mathematik und Englisch

${ }^{8}$ Kontrolliert für Gruppenzugehörigkeit

\section{Abbildung 75. Strukturgleichungsmodelle I (Längsschnitt) zur Erklärung problematischen Computerspielverhaltens (6. Klasse).}




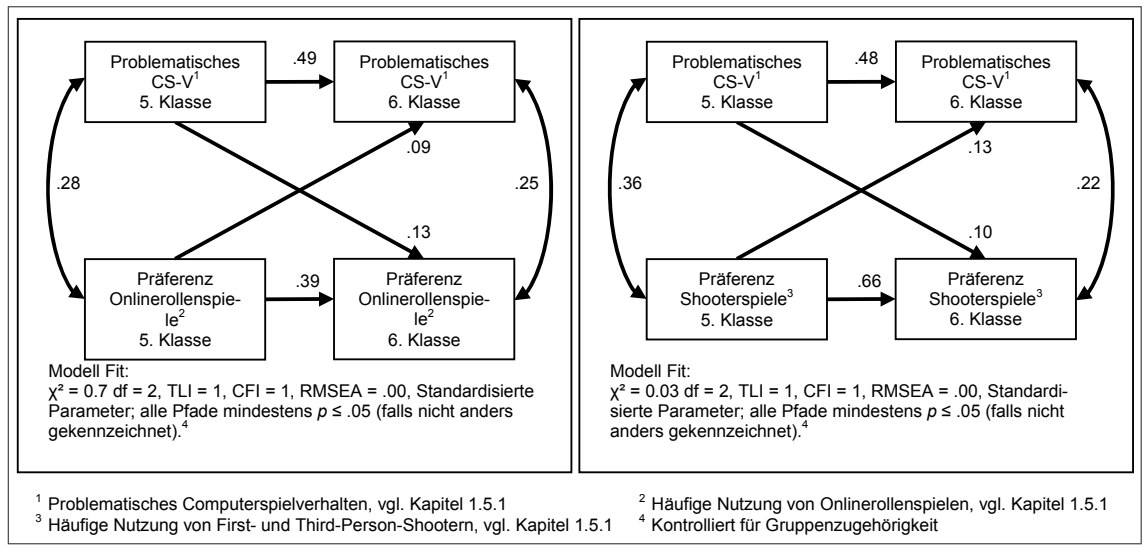

Abbildung 76. Strukturgleichungsmodelle II (Längsschnitt) zur Erklärung problematischen Computerspielverhaltens (6. Klasse).

Die Modelle ${ }^{198}$ (vgl. Abbildung 75 und Abbildung 76) bestätigen zunächst bei insgesamt guter Passungsgüte (depressive Verstimmung: $\chi 2=.03(2, N=739)$, RMSEA $=.00, T L I=1, C F I=1$, Hyperaktivität: $\chi 2=1.4(2, N=739), R M S E A=$. $00, T L I=1, C F I=1$, Verhaltensprobleme mit Gleichaltrigen: $\chi^{2}=3.2(2$, $N=739), R M S E A=.03, T L I=.98, C F I=.99$, Spielen bei realweltlichen Misserfolgen: $\chi^{2}=.02(2, N=739), R M S E A=.00, T L I=1, C F I=1$, Selbstkonzept eigener Fähigkeiten: $\chi 2=3.6(2, N=739), R M S E A=.03, T L I=.99, C F I=.99$, Schulleistung: $\chi 2=.09(2, N=739), R M S E A=.00, T L I=1, C F I=1$, Onlinerollenspiele: $\chi^{2}=.07(2, N=739), R M S E A=.00, T L I=1, C F I=1$, First- und Third-Person-Shooter: $\chi 2=.03(2, N=739), R M S E A=.00, T L I=1, C F I=1)$ jeweils für die fünfte Klasse einen statistisch bedeutsamen mittleren querschnittlichen korrelativen Zusammenhang mit einem erhöhten problematischen Computerspielverhalten $\left(\varphi_{\text {depressive Verstimmung }}=.14, \varphi_{\text {Hyperaktivität }}=.36, \varphi_{\text {Verhaltensprobleme }}=.33, \varphi_{\text {Spielen }}\right.$ bei Misserfolg $=.51, \varphi_{\text {Selbstkonzept }}=-.36, \varphi_{\text {Schulleistung }}=.26, \varphi_{\text {Onlinerollenspiele }}=.28, \varphi_{\text {First- }}$ und Third-Person-Shooter $=.36)$. Die Stabilitäten fallen dabei erwartungsgemäß für die Schulleistungen am höchsten $\left(\beta_{56}=.83\right)$, für die depressive Verstimmung am geringsten $\left(\beta_{56}=.31\right)$ aus. Alle anderen Variablen bewegen sich dazwischen. Die

198 Als Stichprobenauswahl wurden nur Kinder, zu denen zum vierten und fünften Messzeitpunkt Daten vorlagen, in die Analyse aufgenommen $(n=739)$.

Im Modell wurde zudem für das Geschlecht und die Gruppenzugehörigkeit der Kinder kontrolliert. 
Kreuzpfade ${ }^{199}$ zwischen den Messzeitpunkten beantworten schließlich die Frage nach der Wirkrichtung recht eindeutig: Für die meisten Variablen mit Ausnahme der depressiven Verstimmung sowie des Selbstkonzeptes eigener Schulfähigkeiten muss eine Wechselwirkung in annähernd vergleichbarer Höhe angenommen werden. So kann das Vorliegen einer Hyperaktivität $\left(\beta_{56}=.13\right)$, Verhaltensprobleme mit Gleichaltrigen $\left(\beta_{56}=.11\right)$, die Nutzungshäufigkeit von Onlinerollenspielen $\left(\beta_{56}=.09\right)$ sowie die Nutzung von First- und Third-Person-Shootern $\left(\beta_{56}=.13\right)$ späteres problematisches Computerspielverhalten erklären. Gleiches gilt allerdings auch für die Wirkung in umgekehrter Richtung $\left(\beta_{56}\right.$ Hyperaktivität $=$. $10, \beta_{56 \text { Verhaltensprobleme }}=.09, \beta_{56}$ Onlinerollenspiele $=.13, \beta_{56 \text { First- und Third-Person-Shooter }}=$. 10). ${ }^{200}$ Dieser Befund gilt allerdings nur mit Einschränkungen für das Spielen bei realweltlichen Misserfolgen sowie die erbrachten Schulleistungen der Schülerinnen und Schüler, da hier zwar jeweils beide Kreuzpfade statisch bedeutsam werden, jedoch einer deutlich höher ausfällt als der Gegenpfad. So kann Spielen bei realweltlichen Misserfolgen einerseits späteres problematisches Computerspielverhalten erklären $\left(\beta_{56}=.12\right)$, für die Wirkung in umgekehrter Richtung gilt dies aber in höherem Ausmaß $\left(\beta_{56}=.18\right)$. Die erbrachten Schulleistungen können ebenso späteres problematisches Computerspielverhalten erklären $\left(\beta_{56}=.10\right)$, in geringerem Ausma $\beta\left(\beta_{56}=.05\right)$ gilt dies aber auch für die umgekehrte Wirkrichtung. Lediglich ein geringes Selbstkonzept eigener Schulfähigkeiten scheint im Sinne einer Wirkungsannahme kausal mit dem späteren Auftreten von problematischem Computerspielverhalten verknüpft zu sein $\left(\beta_{56}=-.18\right)$. Auf der anderen Seite hängt das Vorliegen einer depressiven Verstimmung kausal mit vorherigem problematischen Computerspielverhalten zusammen $\left(\beta_{56}=.12\right)$.

Zusammengefasst deuten die acht berechneten Strukturgleichungsmodelle darauf hin, dass die meisten Variablen der Persönlichkeit und des sozialen Umfeldes des Erklärungsmodells aus Abbildung 74 gemeinsam mit problematischem Computerspielverhalten auftreten und sich wechselseitig beeinflussen. Nur das frühere Bestehen eines geringen Selbstkonzeptes eigener Schulfähigkeiten sowie zum Teil Schulleistungsprobleme können im Längsschnitt das spätere Auftreten von problematischem Computerspielverhalten kausal erklären. Auf der anderen

199 Zur Verbesserung der Modellgüte wurde in den Modellen eine Korrelation mit problematischem Computerspielverhalten in der sechsten Klasse zugelassen. Inhaltlich bedeutet diese, dass Veränderungen in der einen Variablen mit Veränderungen in der anderen Variable einhergehen. Mit Ausnahme von Selbstkonzept eigener Schulfähigkeiten sowie der Schulleistungen ist diese jeweils statistisch bedeutsam, was in Einklang mit den Befunden zur Wirkrichtung steht.

200 Die Wechselwirkung zeigt sich übrigens auch an den in der sechsten Klasse zugelassenen Korrelation mit problematischem Computerspielverhalten, die jeweils in vergleichbarere Höhe zu denen gemessen in der fünften Klasse ausfallen. Inhaltlich bedeutet dies, dass Veränderungen im Computerspielverhalten mit Veränderungen in der jeweils anderen Variable einhergehen. 
Seite führte problematisches Computerspielverhalten zu einer später auftretenden depressiven Verstimmung.

Um schließlich die Bedeutung früher auffälliger psychosozialer Belastung zu bestimmen, wurde eine lineare Regression (vgl. Tabelle 74) problematischen Computerspielverhaltens (6. Klasse) bei gleichzeitiger Berücksichtigung jener Variablen der Computerspielnutzung, der Persönlichkeit sowie des sozialen Umfeldes, welche sich im Risikomodell aus Abbildung 74 als bedeutsam erwiesen haben, berechnet. Soziale Integration sowie Impulsivität wurden den beiden Konstrukten Verhaltensprobleme mit Gleichaltrigen und Hyperaktivität des SDQ vorgezogen, da erstere auch in der vierten Klasse erhoben wurden und somit Aussagen über einen längeren Zeitraum möglich sind. Es besteht aber sowohl zwischen Hyperaktivität und Impulsivität $\left(\mathrm{r}_{5 \text {.Klasse }}=.48, \mathrm{r}_{6 \text {.Klasse }}=.43\right)$ als auch zwischen soziale Integration und Verhaltensproblemen mit Gleichaltrigen ( $\mathrm{r}_{5 \text {.Klas- }}$ se $=-.50, \mathrm{r}_{6 . \text { Klasse }}=-.57$ ) eine große Inhaltsgleichheit. Es gingen demnach die häufige Nutzung von Onlinerollenspielen sowie von First- und Third-PersonShootern, das Spielen bei realweltlichen Misserfolgen, Impulsivität, soziale Integration, Selbstkonzept eigener Schulfähigkeiten sowie die empfundene elterliche Zuwendung als Prädiktoren in die Analyse ein. In ein erstes Regressionsmodell (Modell I) wurde zunächst neben sämtlichen Variablen der sechsten Klasse das Geschlecht als Prädiktor in die Analyse mit aufgenommen. Wie im Querschnittsmodell (vgl. Abbildung 74) tragen die unterschiedlichen Variablen desselben Messzeitpunktes zunächst in vergleichbarem Ausmaß zur Vorhersage bei. So ist das aktuelle Spielen bei realweltlichem Misserfolg $(\beta=.60)$ auch in diesem Modell die bedeutendste Variable zur Erklärung problematischen Computerspielverhaltens, gefolgt von der aktuellen Nutzung von First- und Third-PersonShootern $(\beta=.09)$ und Impulsivität $(\beta=.09)$, und der aktuellen Nutzung von Onlinerollenspielen $(\beta=.08)$ sowie einem geringen Selbstkonzept eigener Schulfähigkeiten $(\beta=-.08)$. Einzige Ausnahme stellt die soziale Integration dar, welche im Gegensatz zu Verhaltensproblemen mit Gleichaltrigen $(\beta=.11)$ aus Abbildung 74 keine bedeutsame Wirkung entfaltet. In die folgenden Modelle (Modell II und Modell III) wurden neben den aktuellen mediennutzungsspezifischen Risikokorrelaten die Risikokorrelate des Schulkontextes, der Persönlichkeit und des sozialen Umfeldes der fünften bzw. vierten Klasse eingeschlossen. Lediglich das Selbstkonzept eigener Schulfähigkeiten früherer Messzeitpunkte kann neben den aktuellen mediennutzungsspezifischen Risikokorrelaten zur Vorhersage eines problematischen Computerspielverhaltens beitragen.

Im Ergebnis können die berechneten linearen Regressionen die Berechnungen der Strukturgleichungsmodelle aus Abbildung 75 und Abbildung 76 bestätigen: das Selbstkonzept eigener Schulfähigkeiten, welches sich bereits für die Erklärung von Schulleistungsunterschieden als bedeutsam erwiesen hat, scheint auch 
für die Entstehung problematischen Computerspielverhaltens von essenzieller Bedeutung zu sein.

Tabelle 74. Regression problematisches Computerspielverhalten (6. Klasse).

Modell I Modell II $\quad$ Modell III

6. Klasse

Geschlecht

Mediennutzungsspezifische Risikokorrelate

Spielen bei realweltlichen Misserfolgen

Nutzung von First- oder Third-Person-Shootern

Nutzung von Onlinerollenspielen

Risikokorrelate des Schulkontextes

Selbstkonzept eigener Schulfähigkeiten

Soziale Integration

Risikokorrelate der Persönlichkeit

Impulsivität

$-.07 *$

$-.07 *$

$-.07 *$

isikokorrelate des Sozialen Umfelds

Elterliche Zuwendung

$\begin{array}{lll}.60 * * & .61 * * & .63 * * \\ .09 * * & .09 * * & .10^{* *} \\ .08 * * & .09 * * & .09 * *\end{array}$

$-.08 * *$

$-.01^{\mathrm{ns}}$

$.09 * *$

$-.07 *$

5. Klasse

Risikokorrelate des Schulkontextes

Selbstkonzept eigener Schulfähigkeiten

$-.13 * *$

Soziale Integration

Risikokorrelate der Persönlichkeit

Impulsivität

$.02^{\mathrm{ns}}$

Risikokorrelate des Sozialen Umfelds

Elterliche Zuwendung $-.01^{\mathrm{ns}}$

4. Klasse

Risikokorrelate des Schulkontextes

Selbstkonzept eigener Schulfähigkeiten

Soziale Integration

Risikokorrelate der Persönlichkeit

Impulsivität

Risikokorrelate des Sozialen Umfelds

Elterliche Zuwendung

\begin{tabular}{rccc} 
& & & $.01^{\mathrm{ns}}$ \\
Korrigiertes $\boldsymbol{R}^{2}$ & .59 & .58 & .58 \\
$\boldsymbol{p}$ & $<.001$ & $<.001$ & $<.001$ \\
\hline
\end{tabular}

Anmerkung. Abhängige Variable $=$ Skala problematisches Computerspielverhalten. Statistische Unabhängigkeit der Residuen gegeben. Keine Kollinearität gegeben (alle $r \leq .46$, Toleranz $\geq .66$, VIF $\leq 1.5$, keinen auffälligen $C I$ ). Als Stichprobenauswahl wurden nur Kinder der Interventions- und Kontrollklassen, zu denen zum dritten, vierten oder fünften Messzeitpunkt und insgesamt mindestens 
zu zwei Messzeitpunkten Daten vorlagen, aufgenommen $(n=724)$. Die Baselineklassen wurden wegen fehlender Daten für den dritten Messzeitpunkt komplett ausgeschlossen. Spielen bei realweltlichen Misserfolgen: „Ich spiele häufig, wenn es in meinem Leben ansonsten gerade nicht so gut läuft“ (1 - stimmt nicht, 2 - stimmt kaum, 3- stimmt ziemlich, 4 - stimmt genau). Nutzung von First- oder Third-Person-Shootern bzw. Onlinerollenspielen: Schüler, die auf die Frage ,wie häufig spielst du die folgenden Arten von Computer- oder Videospielen“ „nie“ oder „selten“ ankreuzten haben keine Präferenz für dieses Genre. Schüler, die ,sehr oft“ ankreuzten haben eine Präferenz. Haben die Schüler „,manchmal“ oder „oft“ angekreuzt besteht eine Genrepräferenz, wenn kein weiteres Genre öfter gespielt wird (vgl. Kapitel 1.5.1). Selbstkonzept: Selbstkonzept eigener Schulfähigkeiten (FEESS 3-4, Rauer \& Schuck, 2003). Soziale Integration (FEESS 3-4, Rauer \& Schuck, 2003). Hyperaktivität und Verhaltensprobleme mit Gleichaltrigen: Strengths and Difficulties Questionnaire in der deutschen Übersetzung (SDQ-Deu; Goodman, 1997; Klasen et al., 2000, vgl. Kapitel 1.5.2). Impulsivität (IVE, Stadler et al., 2004a, vgl. Kapitel 1.5.2). Elterliche Zuwendung: Skala elterliche Zuwendung (vgl. Kapitel 1.5.1, Tabelle 14). * $p<.05, * * p<.01, \dddot{\imath} p<.10$.

\subsection{Diskussion}

In Kapitel 5.2 wurden zum Thema Computerspielabhängigkeit die folgenden drei Leitfragen, die mit dem Berliner Längsschnitt Medien beantwortet werden sollten, formuliert:

1. Handelt es sich bei Computerspielabhängigkeit um ein reines Jugendphänomen oder können bei im Durchschnitt 11.5-jährigen Schülerinnen und Schülern der fünften Klasse sowie im Durchschnitt 12.5-jährigen Schülerinnen und Schülern der sechsten Klasse, auch bereits Kinder identifiziert werden, die in diesem Alter die klinischen Merkmale einer Computerspielabhängigkeit zeigen?

2. Können ausgehend vom internationalen Forschungsstand bei Betrachtung der Genese einer Computerspielabhängigkeit neben medienspezifischen Risikokorrelaten auch besondere Faktoren des sozialen bzw. schulischen Umfeldes oder der Persönlichkeit ermittelt werden, die Kinder für die Entwicklung einer Computerspielabhängigkeit prädisponieren? Welche Risikokorrelate eignen sich dabei insbesondere zur längsschnittlichen Vorhersage?

3. Können bereits im Kindesalter psychische Auffälligkeiten wie eine depressive Verstimmung oder Hyperaktivität, die später als Komorbiditäten einer Computerspielabhängigkeit berichtet werden, vermehrt bei computerspielabhängigen Kindern beobachtet werden? Gehen diese möglicherweise einer Computerspielabhängigkeit voraus oder sind sie deren Folge?

Im Folgenden sollen die drei Fragenkomplexe jeweils einzeln aufgegriffen und mit der bestehenden Forschungslage in Beziehung gesetzt werden.

Zu 1.) Handelt es sich bei Computerspielabhängigkeit um ein reines Jugendphänomen? Zunächst hat sich die KFN-CSAS auch für eine jüngere Al- 
tersstichprobe als gut geeignet erwiesen, um eine Computerspielabhängigkeit bzw. ein problematisches Computerspielverhalten von einem leidenschaftlich betriebenen, jedoch vergleichsweise weniger problematischen Computerspielverhalten abzugrenzen. Im Vergleich zu anderen Studien, in welchen die KFNCSAS in ihren unterschiedlichen Versionen als Screening-Instrument einer Computerspielabhängigkeit zum Einsatz kam (vgl. Mößle et al., 2007; Rehbein et al., 2009b, 2010; Schmidt, J.-H. et al., 2011), konnten im Berliner Längsschnitt Medien entsprechende Reliabilitätsmaße $(.83<$ Cronbachs $\alpha>.94)$ und Itemkennwerte erzielt werden (vgl. Rehbein et al., 2010; Schmidt, J.-H. et al., 2011). Bei vergleichbaren Mittelwerten und Standardabweichungen wird jedoch ein (etwas) geringerer Anteil der Schülerinnen und Schüler (5. Klasse: $2.7 \%$; 6. Klasse: $4.0 \%$ ) als suchtartige Computerspieler oder -spielerinnen identifiziert (vgl. Tabelle 75).

Tabelle 75. Kennwerte der KFN-CSAS in unterschiedlichen Populationen.

\begin{tabular}{|c|c|c|c|c|c|c|c|c|}
\hline & & $\begin{array}{l}\text { Alter } \\
\text { in } \\
\text { Jah- } \\
\text { ren }\end{array}$ & MW & SD & $\begin{array}{l}\text { unauf- } \\
\text { fällig }\end{array}$ & $\begin{array}{l}\text { gefähr- } \\
\text { det }\end{array}$ & $\begin{array}{l}\text { abhän- } \\
\text { gig }\end{array}$ & $\begin{array}{l}\text { suchtar- } \\
\text { tiges } \\
\text { Spielen }\end{array}$ \\
\hline \multirow{2}{*}{ 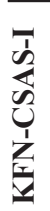 } & $\begin{array}{l}\text { Mößle et al. } \\
(2007)\end{array}$ & 15.1 & 15.81 & 5.8 & $94.9 \%$ & $3.6 \%$ & $1.5 \%$ & $5.1 \%$ \\
\hline & $\begin{array}{l}\text { 5. Klasse } \\
(B L M)\end{array}$ & 11.5 & 15.11 & 4.9 & $97.3 \%$ & $2.0 \%$ & $0.7 \%$ & $2.7 \%$ \\
\hline \multirow{3}{*}{ 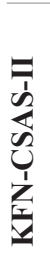 } & $\begin{array}{l}\text { Rehbein et al. } \\
(2009 b)\end{array}$ & 15.6 & 19.81 & 7.4 & $95.5 \%$ & $2.8 \%$ & $1.7 \%$ & $4.5 \%$ \\
\hline & $\begin{array}{l}\text { Schmidt, J.- } \\
\text { H. et al. } \\
(2011)\end{array}$ & $14-19$ & 19.89 & 6.2 & $95.7 \%$ & $3.5 \%$ & $0.9 \%$ & $4.4 \%$ \\
\hline & $\begin{array}{l}\text { 6. Klasse } \\
(B L M)\end{array}$ & 12.5 & 19.48 & 7.5 & $96.0 \%$ & $2.1 \%$ & $1.9 \%$ & $4.0 \%$ \\
\hline
\end{tabular}

Anmerkung. BLM = Berliner Längsschnitt Medien.

Computerspielabhängigkeit scheint somit ein Störungsbild zu sein, welches sich bereits in der (späten) Kindheit entwickelt und in der Adoleszenz deutlich an Bedeutung gewinnt. In Abbildung 77 wurde erstmalig der Versuch unternommen, die Prävalenz einer Computerspielabhängigkeit im Altersverlauf darzustellen (abgetragen sind nur Jungen und junge Männer). Die Daten hierfür kommen aus dem Berliner Längsschnitt Medien, der KFN-Schülerbefragung 2007/2008 (vgl. Rehbein et al., 2010) sowie der KFN-Berufsschülerbefragung (vgl. Rehbein et al., 2011). Zu beachten sind dabei jedoch die teilweise geringen Fallzahlen $\left(\mathrm{N}_{11}\right.$ - 
Jährige $=827 ; \mathrm{N}_{12 \text {-Jährige }}=806 ; \mathrm{N}_{15-\text { Jährige }}=15.168$ (vgl. Rehbein et al., 2009b,

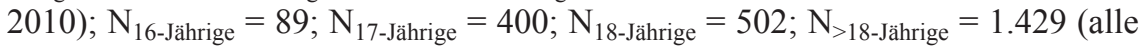
Rehbein et al., 2011)) sowie die teilweise nicht gegebene Repräsentativität, weshalb Abbildung 77 mit gegebener Vorsicht zu betrachten ist. Bis dato wurde allerdings noch in keiner anderen Studie mit einem identischen Screening-Instrument über dieses Altersspektrum das Bestehen einer Computerspielabhängigkeit erhoben. So wurden beispielsweise in den unterschiedlichen Befragungen der Forschergruppe um Grüsser und später Wölfling zwar vergleichbare Altersgruppen untersucht, jedoch immer mit unterschiedlichen bzw. weiterentwickelten Formen der CSV bzw. CSV-K (vgl. Grüsser et al., 2005; Wölfling et al., 2008; Wölfling et al., 2010b). Zu Vergleichszwecken wurden zudem die Lebenszeitprävalenz von ADHD (vgl. Schlack et al., 2007; Edel \& Vollmoeller, 2006) sowie depressiven Störungen (vgl. Mehler-Wex, 2008; Wittchen \& Jacobi, 2006) ebenfalls nur für Jungen und junge Männer abgetragen.

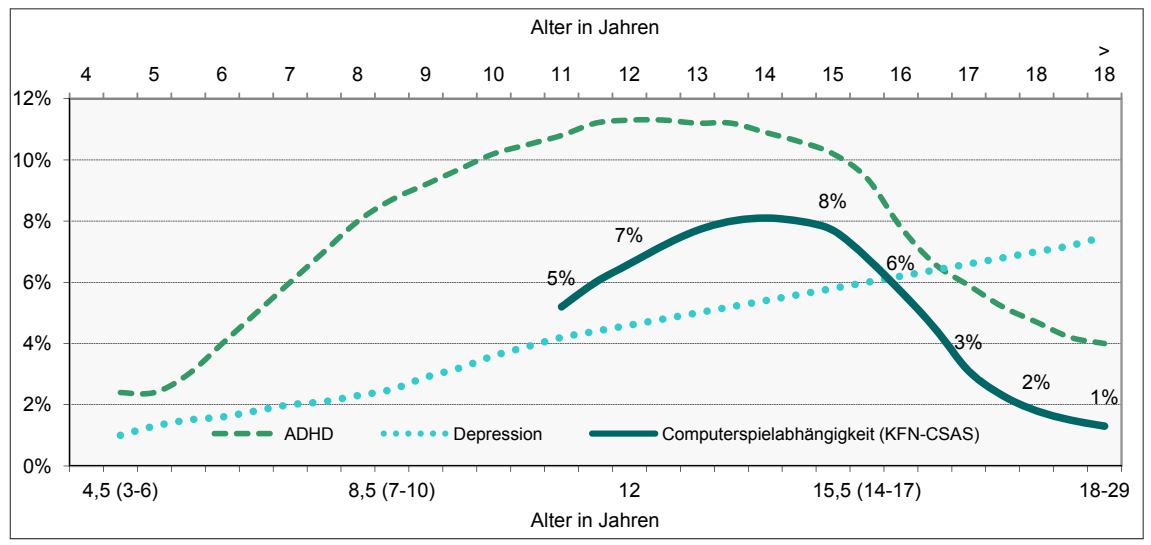

Abbildung 77. Prävalenz (nur Jungen und junge Männer) von ADHD, Depression und Computerspielabhängigkeit in verschiedenen Altersgruppen. Für ADHD und Depression gilt jeweils die unter $\mathrm{X}$-Achse für Computerspielabhängigkeit die obere. ${ }^{201}$

Computerspielabhängigkeit ist demnach kein reines Phänomen der Adoleszenz. Sowohl im späten Kindesalter als auch im jungen Erwachsenenalter können Personen identifiziert werden, die Computerspiele suchtartig nutzen. Im

201 ADHD: vgl. Schlack et al. (2007) sowie Edel \& Vollmoeller (2006). Depressive Störungen: vgl. Mehler-Wex (2008) sowie Wittchen \& Jacobi (2006). Computerspielabhängigkeit: $\mathrm{N}_{11 \text {-Jährige }}=827 ; \mathrm{N}_{12 \text {-Jährige }}=806 ; \mathrm{N}_{15 \text {-Jährige }}=15.168$ (vgl. Rehbein et al., 2009b, 2010); $\mathrm{N}_{16-\text { Jährige }}=89 ; \mathrm{N}_{17 \text {-Jährige }}=400 ; \mathrm{N}_{18 \text {-Jährige }}=502 ; \mathrm{N}_{>18 \text {-Jährige }}$ $=1.429$ (alle Rehbein et al., 2011). 
schlimmsten Fall beginnt das suchtartige Verhalten schon im Kindesalter und geht über die Adoleszenz hinaus. Ein Höhepunkt scheint bei 15-Jährigen zu liegen (die Daten zu den 16-Jährigen sind allerdings aufgrund der geringen Fallzahl nur bedingt vergleichbar). Zum frühen Kindesalter liegen leider keine Vergleichswerte vor. Es ist aber auch hier, wenn man die beobachteten Einflussfaktoren der dritten Klasse als Korrelate zu Grunde legt (vgl. 5.2.3), ein geringer Prozentsatz an suchtartig spielenden Kindern zu erwarten. Im Vergleich hierzu ist ADHD ein Störungsbild, welches bei Jungen bereits frühkindlich beobachtet werden kann, in der späten Kindheit seinen Höhepunkt findet (vgl. Schlack et al., 2007) und bis ins Erwachsenenalter exponentiell abnimmt (vgl. Edel \& Vollmoeller, 2006). Depressive Störungen nehmen hingegen kontinuierlich über die Kindheit zu (vgl. Mehler-Wex, 2008), finden ihren Höhepunkt im jungen Erwachsenalter und verbleiben dann relativ stabil bei einer Prävalenzrate von 7 bis 8 Prozent (vgl. Wittchen \& Jacobi, 2006).

In den Daten des Berliner Längsschnitt Medien zeigt das Störungsbild Computerspielabhängigkeit eine gewisse Stabilität, wobei die Stabilitätswerte jedoch im Vergleich zu Gentile und Kollegen (Gentile et al., 2011) deutlich geringer ausfallen: so wurden 56 Prozent (vgl. 84 \%; Gentile et al., 2011) der Schülerinnen und Schüler, welche in der fünften Klasse als problematisch (gefährdet oder abhängig) klassifiziert wurden dies auch noch in der sechsten Klasse. Weitere drei Prozent (vgl. 1 \%; Gentile et al., 2011) wurden während der einjährigen Befragungsdauer von der fünften zur sechsten Klasse zusätzlich als problematische Computerspieler eingestuft.

Spätestens an dieser Stelle muss eine sehr grundsätzliche Frage aufgeworfen werden: Ist es überhaupt sinnvoll die Kriterien einer Substanzabhängigkeit nach DSM-IV-TR (Saß et al., 2003) bzw. des Abhängigkeitssyndroms nach ICD-10 (Dilling et al., 2006) oder die Kriterien des pathologischen Spielens nach DSMIV-TR (Saß et al., 2003) bzw. ICD-10 (Dilling et al., 2006), welche auf Erwachsene angewendet werden, in ihrer Adaptation für Computerspielabhängigkeit auf das Jugend- oder gar Kindesalter zu übertragen? Zunächst ist das Computerspielen im Gegensatz zu Glücksspielen (welche eher im Erwachsenenalter ihre Verbreitung finden) eine weit verbreitete Freizeitaktivität im Kindes- und Jugendalter, die nicht per se pathologisiert werden darf. Deshalb ist es wichtig, dass kurzzeitige Episoden exzessiven Spielens nicht als Computerspielabhängigkeit klassifiziert werden dürfen. Ebenso sollte ein „Starkes Verlangen“ aufgrund der hohen Attraktivität der Mediennutzung für Kinder und Jugendliche (vgl. Rehbein et al., 2009b) nicht als Kriterium herangezogen werden. Jedoch zeigt sich im Berliner Längsschnitt Medien auch bei einer sehr konservativen Anwendung der diagnostischen Kriterien des Abhängigkeitssyndroms nach ICD-10 (Dilling et al., 2006) ein gewisser Anteil an Kindern, die früh auffällig werden. Dass diese auf- 
fällige Computerspielnutzung unter Fünft- und Sechstklässlern ähnlich wie bei anderen Populationen (vgl. Anderson, C. A. \& Dill, 2000; Walsh, 2000; Cleary et al., 2002; Griffiths, 2002; Tejeiro Salguero \& Bersabe Morán, 2002; Griffiths, 2005; Batthyány et al., 2009; Gentile, 2009; Rehbein et al., 2009b; Choo et al., 2010; Rehbein et al., 2010; Wölfling et al., 2010b; Gentile et al., 2011; Schmidt, J.-H. et al., 2011) tatsächlich den Charakter eines Störungsbildes aufweist, zeigen die dargestellten Zusammenhänge mit Merkmalen schulischer Leistung, schulischer Integration, Persönlichkeit, Familienstruktur sowie individuellen Computerspielpräferenzen. Im Gegensatz zu unauffäligen Computerspielerinnen und Computerspielern weisen diese Kinder eine deutlich erhöhte psychosoziale Belastung auf. Zudem sind psychische Erkrankungen des Kindes- und Jugendalters besonders dann als kritisch zu bewerten, wenn aufgrund dessen bestimmte Entwicklungsphasen nicht bewältigt werden können (vgl. Rehbein et al., 2011). Genau darauf weisen Daten einer Untersuchung von Huang (2006) aber hin, in der internetabhängige Collegestudenten Defizite in der Bewältigung der psychosozialen Entwicklungsstadien „Identität vs. Identitätsdiffusion“ (Adoleszenz) bzw. „Intimität vs. Isolierung“ (frühes Erwachsenalter) nach Eriksson aufwiesen. Aufgrund der gegebenen klinischen Relevanz scheint es also als gerechtfertigt, die Kriterien einer Substanzabhängigkeit bzw. des Abhängigkeitssyndroms nach ICD-10 oder die Kriterien des pathologischen Spielens in ihrer Adaptation für Computerspielabhängigkeit auf das Jugend- oder Kindesalter zu übertragen. ${ }^{202}$

\section{Zu 2.) Gibt es besondere Faktoren der Persönlichkeit oder des sozialen} bzw. schulischen Umfeldes, die Kinder für die Entwicklung einer Computerspielabhängigkeit prädisponieren? Welche Risikokorrelate eignen sich dabei insbesondere zur längsschnittlichen Vorhersage? Zunächst konnte analog zur bestehenden Forschungslage im Berliner Längsschnitt Medien auch für die Schülerinnen und Schüler der sechsten Klasse mit einem problematischem Computerspielverhalten eine Reihe von Risikokorrelaten einer Computerspielabhängigkeit berichtet werden.

Im Schulkontext waren dies schlechtere Schulnoten (vgl. Anderson, C. A. \& Dill, 2000; Walsh, 2000; Rehbein et al., 2009b, 2010; Batthyány et al., 2009; Gentile, 2009; Choo et al., 2010; Gentile et al., 2011; Wölfling et al., 2010b), das häufigere Wiederholen einer Klasse (vgl. Rehbein et al., 2009b, 2010) sowie ein erhöhter Schulabsentismus - insbesondere um zu Spielen - (vgl. Rehbein et al., 2009b, 2010; Choo et al., 2010; Wölfling et al., 2010b). Zudem hatten die Schülerinnen und Schüler mit problematischem Computerspielverhalten im Vergleich

202 Wir scheinen es möglicherweise mit einer Abhängigkeitserkrankung zu tun zu haben, die zumeist schon im Kindes- und Jugendalter manifest wird (vgl. Rehbein et al., 2010; Gentile et al., 2011; Chiu, Lee \& Huang, 2004; ein früher Krankheitsbeginn ist insgesamt für Abhängigkeitserkrankungen nicht ungewöhnlich, vgl. Resch, Mattejat \& Remmschimidt, 2006). 
zu denjenigen mit unproblematischem Computerspielverhalten ein geringeres Selbstkonzept eigener Fähigkeiten und eine stärker negative Schuleinstellung, waren weniger sozial integriert, bekamen weniger oft eine Schullaufbahnempfehlung für das Gymnasium und konnten schließlich auch öfter als Opfer von Gewalt und Mobbing in der Schule identifiziert werden.

Im Bereich der Persönlichkeit und Sozialstruktur kennzeichneten sich die suchtartigen Nutzer zudem durch eine geringere Empathiefähigkeit (vgl. Gentile et al., 2011) sowie durch ein geringeres prosoziales Verhalten und durch allgemeine Verhaltensprobleme (vgl. Choo et al., 2010; Wölfling et al., 2010b). Sie waren deutlich häufiger Scheidungskinder, berichteten häufiger über geringere elterliche Zuwendung und erlebten häufiger Gewalt im Elternhaus, wurden also von ihren Eltern geschlagen oder gar verprügelt. Des Weiteren waren diese Kinder primär (deutsche) Jungen, kamen öfters aus einem Elternhaus mit niedrigem Bildungsniveau und geringem Wohlstand.

Als mediennutzungsspezifische Risikofaktoren einer Computerspielabhängigkeit erwiesen sich neben höheren Computerspielzeiten der problematischen Computerspieler und -spielerinnen (vgl. Tejeiro Salguero \& Bersabe Morán, 2002; Gentile, 2009; Rehbein et al., 2009b, 2010; Choo et al., 2010; Wölfling et al., 2010b; Gentile et al., 2011), das Spielen bei realweltlichem Misserfolg im Sinne eines dysfunktionalen medienbezogenen Copings (vgl. Batthyány et al., 2009; Rehbein et al., 2010; Wölfling et al., 2010b) sowie insbesondere die Nutzung von Onlinerollenspielen (MMORPGs) (vgl. Rehbein et al., 2010; Wölfling et al., 2010b; Schmidt, J.-H. et al., 2011). Zusätzlich kennzeichneten sich die suchtartigen Nutzer durch eine weniger aktive elterliche Medienerziehung sowie durch höhere elterliche Mediennutzungszeiten.

Die gegenseitige Interdependenz sowie die relative Bedeutung der einzelnen Risikokorrelate konnte darüber hinaus in einem Risikomodell problematischen Computerspielverhaltens (vgl. Abbildung 74) veranschaulicht werden. Bei gleichzeitiger Berücksichtigung kam auch bestimmten Computerspielnutzungsmustern für die Ausprägung eines problematischen Computerspielverhaltens eine eigenständige Erklärungskraft zu. Neben der häufigen Nutzung von Onlinerollenspielen sowie der häufigen Nutzung von First- und Third-Person-Shootern, war dies insbesondere die Nutzung von Computerspielen, wenn es im Leben gerade ,nicht so gut läuft“. Weiterhin erwiesen sich das Geschlecht Junge, ein niedriges Selbstkonzept eigener Schulfähigkeiten, Hyperaktivität sowie Verhaltensprobleme mit Gleichaltrigen als geeignete erklärende Variablen für problematisches Computerspielverhalten. Geringe elterliche Zuwendung, erlebte Elterngewalt sowie eine depressive Verstimmung waren in diesem Modell keine bedeutsamen Prädiktoren. 
Neben diesem gemeinsamen Auftreten der obigen Risikokorrelate in der sechsten Klasse, zeigte sich aber auch, dass Kinder, die sich in der fünften oder sechsten Klasse durch ein problematisches Computerspielverhalten kennzeichneten, auch schon besonders früh, d. h. bereits in der dritten Klasse, eine auffällige psychosoziale Belastung haben. So hatten hinsichtlich eines problematischen Computerspielverhaltens auffällige Kinder bereits in der dritten Klasse im Mittel schlechtere Noten in den Fächern Deutsch, Mathematik und Sachkunde als Kinder mit einem unauffälligen Computerspielverhalten. Sie empfanden sich ebenso bereits in der dritten Klasse schlechter in den Klassenverbund sozial integriert als die Vergleichsgruppe der unauffälligen Computerspieler und hatten bereits zu diesem Zeitpunkt ein geringeres Selbstkonzept eigener Schulfähigkeiten.

Darüber hinaus waren Kinder, die in der fünften oder sechsten Klasse als suchtartige Computerspielnutzer identifiziert wurden, deutlich früher mit einem eigenen PC und einer eigenen Spielkonsole im Kinderzimmer ausgestattet (vgl. Choo et al., 2010; Wölfling et al., 2010b) und hatten bereits in der dritten Klasse mehr als doppelt so hohe tägliche Computerspielzeiten als die Vergleichsgruppe der unauffälligen Computerspieler. Fast ein Drittel der Kinder in der Gruppe der abhängigen und gefährdeten Computerspieler berichtete bereits in der dritten Klasse über ein zeitlich exzessives Computerspielverhalten.

Letztlich gaben die in der fünften und sechsten Klasse als auffällig diagnostizierten Computerspieler bereits in der dritten Klasse deutlich seltener an, dass ihre Eltern ihre Computerspielnutzung klar reglementieren und begleiten und berichteten bereits in der dritten Klasse über eine geringere elterliche Zuwendung.

Insgesamt zeichnet sich somit im Berliner Längsschnitt Medien für das Phänomen der Computerspielabhängigkeit das Bild eines bereits besonders früh multipel belasteten (schulische Leistung, schulische Integration, Persönlichkeit, Familienstruktur, Mediennutzung) Kindes (vgl. Anderson, C. A. \& Dill, 2000; Walsh, 2000; Cleary et al., 2002; Griffiths, 2002; Tejeiro Salguero \& Bersabe Morán, 2002; Griffiths, 2005; Batthyány et al., 2009; Gentile, 2009; Rehbein et al., 2009b; Choo et al., 2010; Rehbein et al., 2010; Wölfling et al., 2010b; Gentile et al., 2011; Schmidt, J.-H. et al., 2011). Bei einer genaueren einjährigen längsschnittlichen - jeweils einzelnen - Betrachtung jener Variablen, welche sich in einem Risikomodell problematischen Computerspielverhaltens (vgl. Abbildung 74) als bedeutsam erwiesen hatten (Verhaltensprobleme mit Gleichaltrigen, Spielen bei realweltlichen Misserfolgen, Selbstkonzept eigener Fähigkeiten, häufige Nutzung von Onlinerollenspielen bzw. First- und Third-Person-Shootern; ergänzend wurden auch die Schulleistungen betrachtet), zeigte sich, dass die meisten Variablen der Persönlichkeit und des sozialen Umfeldes gemeinsam mit einem problematischen Computerspielverhalten auftreten und sich wechselseitig beeinflussen. Dementsprechend wären jene Kinder der Stichprobe, welche 
besonders früh durch eine hohe psychosoziale Belastung aufgefallen sind, sehr wahrscheinlich auch häufiger besonders früh als Kinder mit problematischem Computerspielverhalten identifiziert worden. Nur das frühere Bestehen eines geringen Selbstkonzeptes eigener Schulfähigkeiten sowie zum Teil Schulleistungsprobleme konnten im Längsschnitt das spätere Auftreten von problematischem Computerspielverhalten kausal erklären. Der Befund zu den Schulleistungen steht dabei anscheinend im Widerspruch zu Gentile und Kollegen (Gentile et al., 2011), welche geringere Schulleistungen als Folge pathologischer Computerspielnutzung identifizierten. Gentile und Kollegen (Gentile et al., 2011) gehen jedoch trotzt der gefunden Kausalität von einer wechselseitigen Beeinflussung aus, was den Befunden des Berliner Längsschnitt Medien entsprechen würde.

Bei einer gleichzeitigen regressionsanalytischen Berücksichtigung der obigen Variablen in einem zweijährigen Längsschnitt zeigten sich schließlich das Spielen bei realweltlichem Misserfolg sowie das Selbstkonzept eigener Schulfähigkeiten, welches sich bereits für die Erklärung von Schulleistungsunterschieden als bedeutsam erwiesen hat, als erklärende Variablen von essenzieller Bedeutung. Spielen bei realweltlichen Misserfolgen hängt aber direkt mit der erlebten Gewalt im Elternhaus und einer geringen elterlichen Zuwendung zusammen (vgl. Abbildung 74). Dies ist als besonders problematisch zu bewerten, da durch die Nähe zum Konstrukt der dysfunktionalen Stressregulation zu befürchten ist, dass eine Auseinandersetzung mit realweltlichen Problemen stellvertretend in Computerspielen stattfindet. Wird dies im Sinne einer Selbsttherapie verstanden, erscheint ein solches Verhalten nicht nur nachvollziehbar, sondern auch sinnvoll. Verbleibt die „Aufarbeitung“ der Probleme jedoch im Virtuellen, hat dies nur wenig Bedeutung für die Realität, sondern kann vielmehr eine Flucht ins Virtuelle verstärken („,dort werde ich verstanden“, „dort erlebe ich Erfolge“, „dort kann ich mich wehren“) und die Gefahr einer Computerspielabhängigkeit erhöhen. In die gleiche Richtung weisen dabei auch die Zusammenhänge mit einem geringen Selbstkonzept eigener Fähigkeiten. Geht man davon aus, dass dieses auch mit geringeren Selbstwirksamkeitserfahrungen zusammenhängt (vgl. auch kognitive Erklärungsansätze, Kapitel 5.1.4), ist es augenscheinlich und ebenso verständlich warum sich Kinder mit einem geringem Selbstkonzept eigener Fähigkeiten der virtuellen Welt der Computerspiele zuwenden. So kann man sich in diesen seine Erfolgserlebnisse selbst generieren, indem man durch die Auswahl des Spiels, des Levels, etc. für eine optimale Passung zwischen Spiel und sich selbst sorgt. Zudem bekommt man, sei es im Shooter oder im Onlinerollenspiel, ein direktes und unmittelbares Erfolgsfeedback auf die ausgeführten Handlungen, was beispielsweise von den Eltern der betroffenen Kinder zumeist nicht zu erwarten ist. Scheitert man dennoch, kann man entweder einen neuen Versuch starten oder sich einfach ein weiteres Spiel suchen. Beide Konstrukte (geringes Selbstkonzept 
eigener Fähigkeiten sowie Spielen bei realweltlichen Misserfolgen) gepaart können darüber hinaus aber auch dazu führen, dass Erfolge und Anerkennung schließlich im Extremfall nur noch im Virtuellen erzielt werden, was wiederum dieses Verhalten verselbständigen und eine Abhängigkeit vom Computerspiel bestärken kann.

\section{Zu 3.) Können bereits im Kindesalter psychische Auffälligkeiten, die spä-} ter als Komorbiditäten einer Computerspielabhängigkeit berichtet werden, beobachtet werden? Gehen diese einer Computerspielabhängigkeit eher voraus oder sind sie eher deren Folge? Als mögliche Komorbiditäten einer Computerspielabhängigkeit wurden Impulsivität/Hyperaktivität sowie depressive Verstimmung im Berliner Längsschnitt Medien erhoben. Zunächst kennzeichneten sich die suchtartigen Computerspielnutzer der sechsten Klasse im Vergleich zur Gegengruppe durch Aufmerksamkeitsprobleme/Impulsivität (vgl. Hahn \& Jerusalem, 2001a; Gentile, 2009; Rehbein et al., 2009b, 2010; Choo et al., 2010) sowie durch höhere Werte auf der Skala depressive Verstimmung (vgl. Gentile et al., 2011).

Darüber hinaus konnte bei der gemeinsamen Betrachtung der relativen Bedeutung der beiden Komorbiditäten Hyperaktivität und depressive Verstimmung in einem Risikomodell problematischen Computerspielverhaltens (vgl. Abbildung 74) gezeigt werden, dass lediglich der Hyperaktivität, nicht jedoch der depressiven Verstimmung eine eigenständige Erklärungskraft zukommt.

Wie für die obigen Risikokorrelate beschrieben zeigte sich auch für die beiden Komorbiditäten Impulsivität/Hyperaktivität sowie das Bestehen einer depressiven Verstimmung, dass diese neben einem gemeinsamen Auftreten in der sechsten Klasse, auch schon besonders früh, d. h. bereits in der dritten bzw. vierten Klasse bei Kindern, die sich in der fünften oder sechsten Klasse durch ein problematisches Computerspielverhalten kennzeichneten, auffällig werden. So hatten hinsichtlich eines problematischen Computerspielverhaltens auffällige Kinder bereits in der dritten Klasse höhere Werte auf der Skala depressive Verstimmung als Kinder mit einem unauffälligen Computerspielverhalten. Selbiges gilt für die Impulsivitätswerte der vierten Klasse sowie die Hyperaktivitätswerte der fünften Klasse.

Bei wiederum einer genaueren einjährigen längsschnittlichen Betrachtung der beiden Komorbiditäten Hyperaktivität und depressive Verstimmung zeigte sich, dass Hyperaktivität, wie auch die meisten Variablen der Persönlichkeit und des sozialen Umfeldes, bei einer wechselseitigen Beeinflussung gemeinsam mit einem problematischen Computerspielverhalten auftritt. Eine depressive Verstimmung kristallisiert sich aber klar als Folge eines problematischen Computerspielverhaltens. Somit steht dieser Befund, wie der zu den Schulleistungen, zum Teil im Widerspruch zu Gentile und Kollegen (Gentile et al., 2011), welche ebenfalls 
eine Depression als Folge, erhöhte Impulsivität jedoch als Ursache pathologischer Computerspielnutzung identifizierten. Für letzteren Befund ist aber wiederum darauf hinzuweisen, dass dieser Widerspruch bereits dadurch aufgelöst wird, dass die Autoren, den Befunden des Berliner Längsschnitt Medien entsprechend, trotz der gefunden Kausalität bereits von einer wechselseitigen Beeinflussung ausgehen (vgl. Gentile et al., 2011).

Die Ausführungen zu den Komorbiditäten bestärken somit für das Phänomen der Computerspielabhängigkeit das Bild eines bereits besonders früh multipel belasteten Kindes (Hyperaktivität) und verdeutlichen zudem die möglichen Folgen (Depressive Verstimmung). Wie gehen wir nun damit um? Nicht nur die Ausführungen zur Computerspielabhängigkeit zeigen, dass digitale Bildschirmmedien einerseits einen großen Anteil des Alltags unserer Kinder und Jugendlichen (insbesondere der Jungen) bestimmen und von diesen tagtäglich zu Zwecken der Unterhaltung bis hin zur Lebensbewältigung eingesetzt werden. Geschieht dies in einer langfristig funktionalen Art und Weise, steht dem auch nichts entgegen. Bewegt sich das jugendliche Mediennutzungsverhalten andererseits ins Dysfunktionale (auch wenn dies den Jugendlichen mitunter auch funktional erscheinen mag), das zeigen die obigen Ausführungen, ist Vorsicht geboten. Angesichts der suggestiven Kraft der neuen digitalen Medien, insbesondere der Onlinespiele und deren virtuellen Parallelwelten, ist zu erwarten, dass Menschen, beispielsweise mit subklinischen psychischen Auffälligkeiten, auch in eine klinisch relevante Abhängigkeit geführt werden können. Gefährdet sind vor allem Kinder und Jugendliche, deren emotionale Entwicklung besonders anfällig für potenziell schädliche Einflüsse ist. So kann die Einbeziehung langfristiger Folgen ihres Handelns von Kindern und Jugendlichen nur sehr eingeschränkt erwartet werden. Wie eingangs geschildert, ist die Adoleszenz eine Entwicklungsphase, in der sich von den Jugendlichen als funktional wahrgenommene Handlungen als langfristig dysfunktional erweisen können. So ist ein medienabhängiges Verhalten für die Jugendlichen mit ernsthaften Folgen verbunden: Leistungseinbußen in Schule und Gesellschaft, Veränderungen im Verhalten, Veränderungen verhaltensnaher Wahrnehmungs- und Einstellungsschemata, Veränderungen in der sozialen Teilhabe, Beeinträchtigung der körperlichen Gesundheit. Wenn Heranwachsende einen Großteil ihrer Freizeit in der digitalen Welt verbringen oder sogar in eine Computerspielabhängigkeit hineinwachsen, kann ein Scheitern auf dem Weg in ein selbstbestimmtes und unabhängiges Erwachsenleben die Folge sein. Den Eltern kommt dabei nicht nur durch ihr Mediennutzungs- und Erziehungsverhalten eine nicht unbedeutende Rolle zu. Dabei scheinen eine fehlende elterliche Zuwendung sowie das familiäre Mediennutzungsmilieu bedeutende Vermittler zu sein (vgl. Kapitel 2). Daher muss es zu einer gesellschaftlichen Verantwortung werden, den Gefährdungsfaktoren mit adäquaten präventiven 
Angeboten zu begegnen. Kinder und Jugendliche müssen z. B. in ihrer Sozialkompetenz und ihre Fähigkeiten zur Stressbewältigung gestärkt werden und müssen selbstwertförderliche Erfahrungen in der realen Welt erleben können. Dadurch werden allen, ganz besonders aber den computerspielabhängigkeitsgefährdeten Jugendlichen, die Türen für einen selbstbestimmten Gang durch die reale wie mediale Welt eröffnet. 